\title{
An Incompressible Polymer Fluid Interacting with a Koiter Shell
}

\author{
Dominic Breit ${ }^{1}$ (D) Prince Romeo Mensah ${ }^{2,3}$
}

Received: 6 October 2020 / Accepted: 16 January 2021 / Published online: 12 February 2021

(c) The Author(s) 2021

\begin{abstract}
We study a mutually coupled mesoscopic-macroscopic-shell system of equations modeling a dilute incompressible polymer fluid which is evolving and interacting with a flexible shell of Koiter type. The polymer constitutes a solvent-solute mixture where the solvent is modelled on the macroscopic scale by the incompressible Navier-Stokes equation and the solute is modelled on the mesoscopic scale by a Fokker-Planck equation (Kolmogorov forward equation) for the probability density function of the bead-spring polymer chain configuration. This mixture interacts with a nonlinear elastic shell which serves as a moving boundary of the physical spatial domain of the polymer fluid. We use the classical model by Koiter to describe the shell movement which yields a fully nonlinear fourth order hyperbolic equation. Our main result is the existence of a weak solution to the underlying system which exists until the Koiter energy degenerates or the flexible shell approaches a self-intersection.
\end{abstract}

Keywords Incompressible Navier-Stokes-Fokker-Planck system · FENE model · Fluid-structure interaction · Koiter shell

Mathematics Subject Classification 76Nxx · 76N10 · 35Q30 · 35Q84 · 82D60

Communicated by Eliot Fried.

The authors would like to thank S. Schwarzacher and E. Süli for valuable suggestions.

$\bowtie$ Dominic Breit

d.breit@hw.ac.uk

Prince Romeo Mensah

p.mensah@imperial.ac.uk; romeo.mensah@gssi.it

1 Department of Mathematics, Heriot-Watt University, Edinburgh EH14 4AS, UK

2 Department of Mathematics, Imperial College, London SW7 2AZ, UK

3 Gran Sasso Science Institute, Viale F. Crispi, 7, 67100 L’Aquila, Italy 


\section{Introduction}

On the one hand, fluid-structure interactions are common physical phenomena yet mathematically challenging problems with applications in aeroelasticity (Dowell 2015), biomechanics (Bodnár et al. 2014) and hydrodynamics (Chakrabarti 2002) amongst others. On the other hand, the huge industrial application of the interactions between polymer molecules and fluids such as in the production of paints, lubricants, plastics as well as in the processing of food stuff (Bird et al. 1987), makes the analysis of polymeric fluids very important. Therefore, from a mathematical, physical and commercial point-of-view, the analysis of the mutual interaction of all three elements, i.e. fluid, structure and polymer molecules is crucial.

We consider in this work, the evolution of a dilute three-dimensional incompressible polymeric fluid in a spatial domain that is changing with respect to time. The displacement of the boundary is prescribed via the two-dimensional mid-section of the flexible Koiter shell whose energy is a nonlinear function of the first and second fundamental forms of the moving boundary. We prove the existence of a weak solution to the coupled fluid-kinetic system, given by the incompressible NavierStokes-Fokker-Planck sytem, which is interacting with an elastic Koiter shell. The existence time is only restricted once the shell approaches a self-intersection or the Koiter energy degenerates.

Existence of a solution to the Fokker-Planck equation for a given solenoidal velocity field incorporating the center-of-mass diffusion term has been established by El-Kareh and Leal (1989) independently of the Deborah number. The incompressible NavierStokes-Fokker-Planck system for polymeric fluids including center-of-mass diffusion has been studied considerably. See, for example, the works by Barrett et al. (2005), Barrett and Süli (2007, 2008, 2011, 2012a, b), as well as by Gwiazda et al. (2018) and Lukáčová-Medvidová et al. (2017) for the kinetic Peterlin model with a nonlinear spring law for an infinitely extensible spring. All these results derive global-in-time weak solutions for variations of the incompressible Navier-Stokes equations coupled with the Fokker-Planck equation. On the other hand, a unique local-in-time strong solution for the center-of-mass system was first shown to exist by Renardy (1991). Unfortunately, Renardy (1991) excludes the physically relevant FENE dumbbell models. The local theory was then revisited by Jourdain et al. (2004) for the stochastic FENE model for the simple Couette flow and by E et al. (2004) who analysed the incompressible Navier-Stokes equation coupled with a system of SDEs describing the configuration of the spring. The corresponding deterministic system, where instead the incompressible Navier-Stokes equations are coupled with the Fokker-Planck equation, was studied by Li et al. (2004) and Zhang and Zhang (2006). Constantin proved the existence of Lyapunov functionals and smooth solutions in Constantin (2005) and then derived global-in-time strong solutions for the 2-D system in Constantin et al. (2007) together with Fefferman, Titi \& Zarnescu.

The analysis is significantly harder without center-of-mass diffusion since the Fokker-Planck equation becomes a degenerate parabolic equation which behaves like an hyperbolic equation in the space-time variable. A global weak solution result to the incompressible Navier-Stokes-Fokker-Planck system for the FENE dumbbell model without center-of-mass diffusion was recently achieved in the seminal paper (Mas- 
moudi 2013) by Masmoudi. The main difficulty is to pass to the limit in the drag term of the Fokker-Planck equation which does not have any obvious compactness properties. Earlier global weak solution results without center-of-mass diffusion include the work by Lions and Masmoudi (2000, 2007) who studied the corotational case, and Otto and Tzavaras (2008) who studied weak solutions for the stationary system. Masmoudi (2008) also constructed a local-in-time strong solution to the incompressible NavierStokes-Fokker-Planck system for the FENE dumbbell model without center-of-mass diffusion in Masmoudi (2008). Furthermore, the solution is global near equilibrium, see also Klainerman and Majda (1981). Further results on local strong solutions where proved by Luo and Yin (2017) and Breit and Mensah (2018).

With respect to fluid-structure problems, the analysis of weak solutions to incompressible viscous fluids interacting with lower-dimensional linear elastodynamic equations has been studied by Chambolle et al. (2005), by Grandmont (2008), Hundertmark-Zaušková et al. (2016), Lengeler and Růžička (2014) and by Muha and Čanić $(2014,2013)$, just to list a few. In particular, the existence of a weak solution for the three-dimensional viscous incompressible fluid modelled by the Navier-Stokes equations which is interacting with a flexible elastic plate located on one part of the fluid boundary was shown by Chambolle et al. (2005). This solution exists so long as the moving part of the structure does not touch the fixed part of the fluid boundary. By using a singular limit argument, the existence of a weak solution to the incompressible Navier-Stokes equation coupled with a plate in flexion was constructed by Grandmont (2008) as the coefficient modelling the viscoelasticity of the plate tends to zero. In Hundertmark-Zaušková et al. (2016), Hundertmark-Zaušková et al. studied the existence of a weak solution to a power-law viscosity fluid-structure interaction problem for shear-thickening flows. Again, the solution exists until a contact of the elastic boundary with a fixed boundary part is made. Lengeler and Růžička also studied in Lengeler and Růžička (2014), the interaction of an incompressible Newtonian fluid, modelled by the Navier-Stoke equation, with a linear elastic shell of Koiter. Here, the middle surface of the shell serves as the mathematical boundary of the three-dimensional fluid domain. The weak solution is shown to exist so long as the magnitude of the shell's displacement stays below a bound that rules out self-intersection. In Muha and Čanić (2013) however, Muha and Čanić use a semi-discrete, operator splitting numerical scheme to show the existence of a weak solution to a fluid-structure coupled system governed by the two-dimensional incompressible Navier-Stokes equations, while the elastodynamics of the cylindrical wall is modelled by the one-dimensional cylindrical linear Koiter shell model. The solution exists as long as the cylinder radius is greater than zero. A similar existence result as Muha and Čanić (2013) was shown in Muha and Čanić (2014) by the same authors where now, the elastodynamics of the cylinder wall is governed by the one-dimensional linear wave equation modelling the thin structural layer, and by the two-dimension equations of linear elasticity modelling the thick structural layer. Further fluid-structure interaction results includes the work Ignatova et al. (2017) where they construct a small data global solution for the motion of an elastic body inside an incompressible fluid. Boulakia et al. (2019) also considers the situation where the elastic structure is immersed in the fluid and the whole system is confined into a general three-dimensional bounded smooth domain. Well-posedness and stability results for a fluid-structure interaction model with interior damping and 

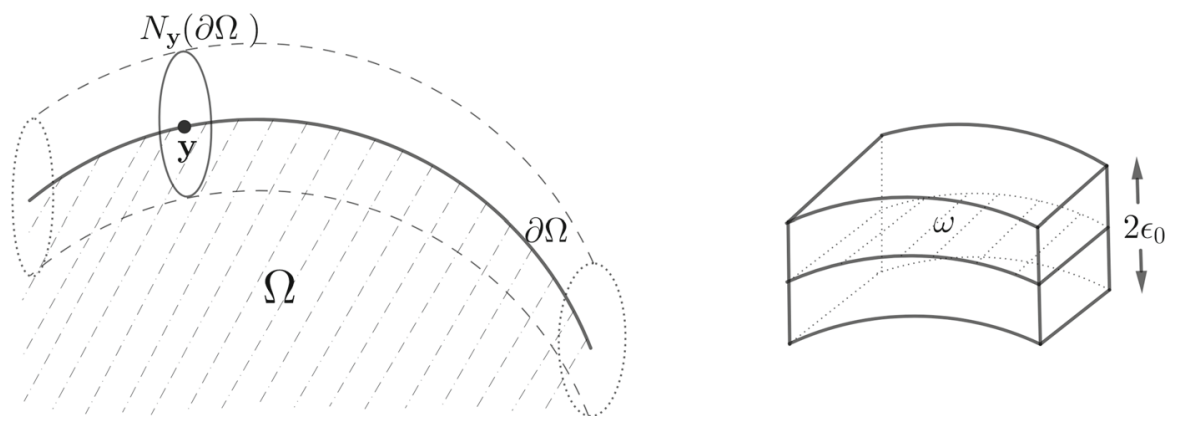

Fig. 1 Left: A tubular neighbourhood of a shell $\partial \Omega$ is represented by the bended cylinder. Right: A macroscopic view of a tiny section of the shell $\partial \Omega$ with thickness $2 \epsilon_{0}>0$. With an abuse of notation, we identify points $\mathbf{y} \in \partial \Omega$ on the 3 -d shell, with points on the middle surface $\mathbf{y} \in \omega$ which is a 2 -d submanifold

delay in the structure is studied by Peralta and Kunisch (2019). As far as we know, the only result on the analysis of weak solutions to fluid-structure interaction, where the original Koiter model (to be described below in Sect.1.2) with a leading order nonlinear shell energy is considered, is the recent paper Muha and Schwarzacher (2019).

Mathematical results concerning the interaction of a polymeric fluid with a flexible structure are, however, still missing in the literature. In this article, we aim to close this gap and initiate a corresponding analysis. In the following, we will describe the model in detail.

\subsection{Elastic Shell}

We are interested in the mathematical analysis of a polymer fluid evolving in a spatial domain with a moving shell. For this reason, we first describe this spatial geometry before we state the equations of motion. Following Lee (2013), we let $\Omega \subset \mathbb{R}^{3}$ be an open, bounded, nonempty and connected reference domain with an elastic shell $\omega \times\left(-\epsilon_{0}, \epsilon_{0}\right) \subset \mathbb{R}^{3}$ of thickness $2 \epsilon_{0}>0$ and a middle surface $\omega$. The boundary $\partial \Omega$ is assumed to be of class $C_{\mathbf{x}}^{4}$. Assume that the movement of the shell $\partial \Omega$ is in the direction of the outer unit normal $\boldsymbol{v}$ (we shall give a precise construction of this normal vector later in Sect. 1.2). Now denote the normal bundle of $\partial \Omega$ by

$$
N(\partial \Omega)=\left\{(\mathbf{y}, \mathbf{z}) \in \mathbb{R}^{3 \times 3}: \mathbf{y} \in \partial \Omega, \quad \mathbf{z} \in N_{\mathbf{y}}(\partial \Omega)\right\}
$$

where $N_{\mathbf{y}}(\partial \Omega)$ is the $(3-2)$-dimensional normal space to $\partial \Omega$ at $\mathbf{y}$ consisting of all vectors orthogonal to the tangent space $T_{\mathbf{y}}(\partial \Omega)$ with respect to the Euclidean dot product. Simply put, $N(\partial \Omega)$ consists of all vectors normal to $\partial \Omega$ and by Lee (2013, Theorem 6.23), $N(\partial \Omega)$ is an embedded 3-dimensional submanifold of $\mathbb{R}^{3 \times 3}$. Consequently, $\partial \Omega$ has a tubular neighbourhood, $S_{L}:=\left\{\mathbf{x} \in \mathbb{R}^{3}: \operatorname{dist}(\mathbf{x}, \partial \Omega)<L\right\}$ for some $L>0$, see Fig. 1 .

Given the outer unit normal $\boldsymbol{v}$ of $\partial \Omega$, one can construct a special affine mapping known as the Hanzawa transform, see Lengeler (2011, Section 2), which will be used below to relate the fixed domain $\Omega$ to a moving one. It is defined in terms of the 
mapping

$$
\Lambda: \partial \Omega \times(-L, L) \rightarrow S_{L}, \quad \Lambda(\mathbf{y}, s)=\mathbf{y}+s \boldsymbol{v}(\mathbf{y})
$$

There is a maximal $L>0$ such that $\Lambda$ is a $C^{3}$-diffeomorphism with inverse

$$
\Lambda^{-1}: S_{L} \rightarrow \partial \Omega \times(-L, L), \quad \Lambda^{-1}(\mathbf{x})=(\mathbf{y}(\mathbf{x}), s(\mathbf{x}))
$$

where $s(\mathbf{x})=(\mathbf{x}-\mathbf{y}(\mathbf{x})) \cdot \boldsymbol{v}(\mathbf{y}(\mathbf{x}))$, cf. Lee (2013, Theorem 6.24). A detailed construction of the Hanzawa transform can be found in Lengeler (2011) but for the sake of completeness, we summarize the construction below.

To begin with, for any specific instant of time $t \in \bar{I}:=\overline{(0, T)}$ where $T>0$, we consider a $C_{\mathbf{x}}^{3}$-function $\eta(t, \cdot): \partial \Omega \rightarrow(-L, L)$ and define the following open set

$$
\Omega_{\eta(t)}:=\Omega \backslash S_{L} \cup\left\{\mathbf{x} \in S_{L}: s(\mathbf{x})<\eta(t, \mathbf{y}(\mathbf{x}))\right\} .
$$

Let $\boldsymbol{v}_{\eta(t)}$ and $\mathrm{d} \mathbf{y}_{\eta(t)}$ be the outer unit normal and the surface measure of $\partial \Omega_{\eta(t)}$ respectively. This function $\eta$ is further assumed to be continuous in time so that $\eta \in C(\bar{I} \times \partial \Omega)$ and we have that

$$
\|\eta\|_{L^{\infty}(I \times \partial \Omega)}<L .
$$

Moving on, we define a $C_{\mathbf{x}}^{3}$-diffeomorphism $\Psi_{\eta(t)}: \bar{\Omega} \rightarrow \bar{\Omega}_{\eta(t)}$ piecewise as

$$
\begin{aligned}
\boldsymbol{\Psi}_{\eta(t)}: \Omega \backslash S_{L} & \rightarrow \bar{\Omega}_{\eta(t)}, \quad \boldsymbol{\Psi}_{\eta(t)}(\mathbf{x})=\mathbf{x}, \\
\boldsymbol{\Psi}_{\eta(t)}: \bar{\Omega} \cap S_{L} & \rightarrow \bar{\Omega}_{\eta(t)}, \quad \boldsymbol{\Psi}_{\eta(t)}(\mathbf{x})=\mathbf{x}+\boldsymbol{v}(\mathbf{y}(\mathbf{x})) \eta(t, \mathbf{y}(\mathbf{x})) \beta(s(\mathbf{x}) / L) \\
& =\mathbf{y}(\mathbf{x})+\boldsymbol{v}(\mathbf{y}(\mathbf{x}))[s(\mathbf{x})+\eta(t, \mathbf{y}(\mathbf{x})) \beta(s(\mathbf{x}) / L)]
\end{aligned}
$$

where $\beta \in C^{\infty}(\mathbb{R})$ is a real-valued function which is zero in a neighbourhood of -1 and one in a neighbourhood of 0 . For the mapping $\boldsymbol{\Psi}_{\eta(t)}$ to have a continuously differentiable spatial inverse, we assume that $\left|\beta^{\prime}(s)\right|<L /|\eta(\mathbf{y})|$ for all $s \in[-1,0]$ and all $\mathbf{y} \in \partial \Omega$. The boundary mapping is also a $C_{\mathbf{x}}^{3}$-diffeomorphism defined as

$$
\boldsymbol{\Phi}_{\eta(t)}:=\left.\boldsymbol{\Psi}_{\eta(t)}\right|_{\partial \Omega}: \partial \Omega \rightarrow \partial \Omega_{\eta(t)}, \quad \boldsymbol{\Phi}_{\eta(t)}(\mathbf{y})=\mathbf{y}+\boldsymbol{v}(\mathbf{y}) \eta(t, \mathbf{y})
$$

for every time $t \in \bar{I}$ with inverse $\boldsymbol{\Phi}_{\eta(t)}^{-1}(\mathbf{x})=\mathbf{y}(\mathbf{x})$.

The diffeomorphisms $\boldsymbol{\Phi}_{\eta}$ and $\boldsymbol{\Psi}_{\eta}$ constructed above, and thus the deformed shell $\bar{\Omega}_{\eta(t)}$, satisfy various continuity and embedding properties. A detailed analyses of these can be found in Lengeler (2011); Lengeler and Růžička (2014).

To summarize, if we denote the closure of the deformed spacetime cylinder $\cup_{t \in I}\{t\} \times \Omega_{\eta(t)} \subset \mathbb{R}^{4}$ by $\bar{I} \times \bar{\Omega}_{\eta(t)}$, then the mapping

$$
\Psi_{\eta}: \bar{I} \times \bar{\Omega} \rightarrow \bar{I} \times \bar{\Omega}_{\eta(t)}, \quad(t, \mathbf{x}) \mapsto\left(t, \Psi_{\eta(t)}(\mathbf{x})\right)
$$


preserves the portion of the original spacetime cylinder $\bar{I} \times \bar{\Omega}$ that lies outside the tubular neigbourhood $S_{L}$ and deforms the residual portion of the original space-time cylinder according to the mapping (1.3). The restriction of $\Psi_{\eta}$ to the boundary is given by the mapping

$$
\boldsymbol{\Phi}_{\eta}: \bar{I} \times \partial \Omega \rightarrow \bar{I} \times \partial \Omega_{\eta(t)}, \quad(t, \mathbf{x}) \mapsto\left(t, \boldsymbol{\Phi}_{\eta(t)}(\mathbf{x})\right)
$$

according to the rule (1.4).

We now move on to give a precise description of the evolution of the shell and its associated energy below.

\subsection{Koiter Shell Energy and Equation of Motion}

The polymer fluid we wish to model is assumed to interact with a Koiter shell $\omega \times$ $\left(-\epsilon_{0}, \epsilon_{0}\right) \subset \mathbb{R}^{3}$. Here, $\omega \subset \mathbb{R}^{2}$ is the middle surface of the shell, recall Fig. 1, and $2 \epsilon_{0}>0$ is the thickness of $\partial \Omega$ and for simplicity, we take $\omega=\mathbb{R}^{2} \backslash \mathbb{Z}^{2}$ to be the flat torus. We emphasis that this periodic assumption on $\omega$ is not at all restrictive and everything we do subsequently can be replicated for a general $\omega$. Following Ciarlet and Roquefort (2001), we suppose that $\partial \Omega$ can be parametrised by a smooth injective mapping $\boldsymbol{\varphi}: \omega \rightarrow \mathbb{R}^{3}$ such that for all points $\mathbf{y}=\left(y_{1}, y_{2}\right) \in \omega$, the pair of vectors $\partial_{i} \boldsymbol{\varphi}(\mathbf{y}), i=1,2$, are linearly independent where, $\partial_{i}:=\partial / \partial_{y_{i}}$. Simply put, $\boldsymbol{\varphi}$ is an injective map on the mid-section of the shell of the domain $\Omega$. This vector pair $\left[\partial_{1} \varphi(\mathbf{y}), \partial_{2} \varphi(\mathbf{y})\right]$ is the covariant basis of the tangent plane to the middle surface $\varphi(\omega)$ of the reference configuration at each point $\varphi(\mathbf{y})$ and

$$
\boldsymbol{v}(\mathbf{y})=\frac{\partial_{1} \varphi(\mathbf{y}) \times \partial_{2} \varphi(\mathbf{y})}{\left|\partial_{1} \varphi(\mathbf{y}) \times \partial_{2} \varphi(\mathbf{y})\right|}
$$

is a well-defined unit vector normal to the surface $\varphi(\omega)$ at $\varphi(\mathbf{y})$. The area measure along the surface $\varphi(\omega)$ is $\mathrm{d} \mathbf{y}_{v}:=\left|\partial_{1} \varphi(\mathbf{y}) \times \partial_{2} \varphi(\mathbf{y})\right| \mathrm{d} \mathbf{y}$. We now assume that the shell (and in particular, its middle surface) only deforms along the normal direction with a displacement field $\eta \boldsymbol{v}: I \times \omega \rightarrow \mathbb{R}^{3}$ where $\eta: I \times \omega \rightarrow \mathbb{R}$ is considerably smooth. Then, we can parametrized the deformed boundary by the following coordinates

$$
\boldsymbol{\varphi}_{\eta}(t, \mathbf{y})=\boldsymbol{\varphi}(\mathbf{y})+\eta(t, \mathbf{y}) \boldsymbol{v}(\mathbf{y}), \quad t \in I, \mathbf{y} \in \omega,
$$

yielding the deformed middle surface $\varphi_{\eta}(t, \omega)$. Now for

$$
\partial_{i} \boldsymbol{\varphi}_{\eta}(t, \mathbf{y})=\partial_{i} \boldsymbol{\varphi}(\mathbf{y})+\partial_{i} \eta(t, \mathbf{y}) \boldsymbol{v}(\mathbf{y})+\eta(t, \mathbf{y}) \partial_{i} \boldsymbol{v}(\mathbf{y}), \quad i=1,2,
$$

the covariant components of the first fundamental form of the deformed middle surface $\varphi_{\eta}(t, \omega)$ is given by

$$
\partial_{i} \boldsymbol{\varphi}_{\eta}(t, \mathbf{y}) \cdot \partial_{j} \boldsymbol{\varphi}_{\eta}(t, \mathbf{y})=\partial_{i} \boldsymbol{\varphi}(\mathbf{y}) \cdot \partial_{j} \boldsymbol{\varphi}(\mathbf{y})+G_{i j}(\eta)
$$


where

$$
\begin{aligned}
G_{i j}(\eta): & \partial_{i} \eta(t, \mathbf{y}) \partial_{j} \eta(t, \mathbf{y})+\eta(t, \mathbf{y})\left[\partial_{i} \boldsymbol{\varphi}(\mathbf{y}) \cdot \partial_{j} \boldsymbol{v}(\mathbf{y})+\partial_{j} \varphi(\mathbf{y}) \cdot \partial_{i} \boldsymbol{v}(\mathbf{y})\right] \\
& +\eta^{2}(t, \mathbf{y}) \partial_{i} \boldsymbol{v}(\mathbf{y}) \cdot \partial_{j} \boldsymbol{v}(\mathbf{y})
\end{aligned}
$$

are the covariant components of the 'modified' change of metric tensor $\mathbb{G}(\eta)$. The normal (which is not a unit vector) to the deformed middle surface $\varphi_{\eta}(t, \omega)$ at the point $\boldsymbol{\varphi}_{\eta}(t, \mathbf{y})$ is then given by

$$
\begin{aligned}
\boldsymbol{v}_{\eta}(t, \mathbf{y})= & \partial_{1} \boldsymbol{\varphi}_{\eta}(t, \mathbf{y}) \times \partial_{2} \boldsymbol{\varphi}_{\eta}(t, \mathbf{y})=\boldsymbol{v}(\mathbf{y})\left|\partial_{1} \varphi(\mathbf{y}) \times \partial_{2} \varphi(\mathbf{y})\right|+\partial_{2} \eta(t, \mathbf{y})\left(\partial_{1} \varphi(\mathbf{y}) \times \boldsymbol{v}(\mathbf{y})\right. \\
& \left.+\eta(t, \mathbf{y}) \partial_{1} \boldsymbol{v}(\mathbf{y}) \times \boldsymbol{v}(\mathbf{y})\right)+\partial_{1} \eta(t, \mathbf{y})\left(\boldsymbol{v}(\mathbf{y}) \times \partial_{2} \varphi(\mathbf{y})+\eta(t, \mathbf{y}) \boldsymbol{v}(\mathbf{y}) \times \partial_{2} \boldsymbol{v}(\mathbf{y})\right) \\
& +\eta(t, \mathbf{y})\left(\partial_{1} \varphi(\mathbf{y}) \times \partial_{2} \boldsymbol{v}(\mathbf{y})+\partial_{1} \boldsymbol{v}(\mathbf{y}) \times \partial_{2} \varphi(\mathbf{y})\right)+\eta^{2}(t, \mathbf{y})\left(\partial_{1} \boldsymbol{v}(\mathbf{y}) \times \partial_{2} \boldsymbol{v}(\mathbf{y})\right)
\end{aligned}
$$

and

$$
R_{i j}^{\sharp}(\eta):=\frac{\partial_{i j} \boldsymbol{\varphi}_{\eta}(t, \mathbf{y}) \cdot \boldsymbol{v}_{\eta}(t, \mathbf{y})}{\left|\partial_{1} \boldsymbol{\varphi}(\mathbf{y}) \times \partial_{2} \boldsymbol{\varphi}(\mathbf{y})\right|}-\partial_{i j} \boldsymbol{\varphi}(\mathbf{y}) \cdot \boldsymbol{v}(\mathbf{y}), \quad i, j=1,2,
$$

are the covariant components of the change of curvature tensor $\mathbb{R}^{\sharp}(\eta)$. The elastic energy $K(\eta):=K(\eta, \eta)$ of the deformation is then given by

$$
\begin{aligned}
K(\eta) & =\frac{1}{2} \epsilon_{0} \int_{\omega} \mathbb{C}: \mathbb{G}(\eta) \otimes \mathbb{G}(\eta) \mathrm{d} \mathbf{y}+\frac{1}{6} \epsilon_{0}^{3} \int_{\omega} \mathbb{C}: \mathbb{R}^{\sharp}(\eta) \otimes \mathbb{R}^{\sharp}(\eta) \mathrm{d} \mathbf{y} \\
& :=\sum_{i, j, k, l=1}^{2} \frac{1}{2} \epsilon_{0} \int_{\omega} C^{i j k l} G_{k l}(\eta) G_{i j}(\eta) \mathrm{d} \mathbf{y}+\frac{1}{6} \epsilon_{0}^{3} \int_{\omega} C^{i j k l} R_{k l}^{\sharp}(\eta) R_{i j}^{\sharp}(\eta) \mathrm{d} \mathbf{y}
\end{aligned}
$$

where $\mathbb{C}=\left(C^{i j k l}\right)_{i, j, k, l=1}^{2}$ is a fourth-order tensor whose entries are the contravariant components of the shell elasticity, see Ciarlet (2005, Page 162). We remark that for simplicity, we have normalized the measure $\mathrm{dy}$ in (1.5) which should have actually been the weighted measure $\mathrm{d} \mathbf{y}_{v}:=\left|\partial_{1} \varphi(\mathbf{y}) \times \partial_{2} \varphi(\mathbf{y})\right| \mathrm{d} \mathbf{y}$ with the non-zero weight $\left|\partial_{1} \varphi(\mathbf{y}) \times \partial_{2} \varphi(\mathbf{y})\right|$, see Roquefort (2001). Next, given the following geometric quantity

$$
\begin{aligned}
\gamma(\eta):=1 & +\frac{\eta}{\left|\partial_{1} \varphi(\mathbf{y}) \times \partial_{2} \varphi(\mathbf{y})\right|}\left[\boldsymbol{v}(\mathbf{y}) \cdot\left(\partial_{1} \varphi(\mathbf{y}) \times \partial_{2} \boldsymbol{v}(\mathbf{y})+\partial_{1} \boldsymbol{v}(\mathbf{y}) \times \partial_{2} \varphi(\mathbf{y})\right)\right] \\
& +\frac{\eta^{2}}{\left|\partial_{1} \varphi(\mathbf{y}) \times \partial_{2} \varphi(\mathbf{y})\right|} \boldsymbol{v}(\mathbf{y}) \cdot\left(\partial_{1} \boldsymbol{v}(\mathbf{y}) \times \partial_{2} \boldsymbol{v}(\mathbf{y})\right),
\end{aligned}
$$

one deduces the $W^{2,2}(\omega)$-coercivity of the Koiter energy (1.5) as long as $\gamma(\eta) \neq 0$. This is the case if $\|\eta\|_{L^{\infty}(\omega)} \leq \tilde{L}$ for some $\tilde{L}>0$ depending on the geometry of $\Omega$. Further details can be found in Muha and Schwarzacher (2019, Lemma 4.3 and Remark 4.4). Without loss of generality, we assume that $L \leq \tilde{L}$, where $L$ is the threshold for self-intersection introduced in Sect. 1.1. Finally, we remark that the 
Koiter energy is continuous on $W^{2, p}(\omega)$ for all $p>2$ due to the Sobolev embedding $W^{2, p}(\omega) \hookrightarrow W^{1, \infty}(\omega)$.

If the mass density of $\omega$ is $\epsilon_{0} \rho_{S}$ where $\rho_{S}>0$ is a constant, and we simply denote the $L^{2}$-gradient of $K$ by $K^{\prime}$ (which is to be interpreted in sense that $K^{\prime}(\eta) \xi=$ $\left\langle K^{\prime}(\eta), \xi\right\rangle$ for any $\zeta$ in the dual space of $\left.\eta\right)$, then the evolution of the shell is modelled by (later on, we assume for simplicity that $\epsilon_{0} \rho_{S}=1$ )

$$
\epsilon_{0} \rho_{S} \partial_{t}^{2} \eta+K^{\prime}(\eta)=g+\mathbf{F} \cdot \boldsymbol{v}
$$

in $I \times \omega$ subject to the following initial and boundary conditions

$$
\begin{array}{lr}
\eta(0, \cdot)=\eta_{0}, \quad \partial_{t} \eta(0, \cdot)=\eta_{1} & \text { in } \omega \\
\mathbf{u}(t, \boldsymbol{\varphi}(\mathbf{y})+\eta(t, \mathbf{y}) \boldsymbol{v}(\mathbf{y}))=\partial_{t} \eta(t, \mathbf{y}) \boldsymbol{v}(\mathbf{y}) & \text { on } I \times \omega
\end{array}
$$

where $\eta_{0}, \eta_{1}: \omega \rightarrow \mathbb{R}$ are given functions and where in (1.7), the function $g: I \times \omega \rightarrow$ $\mathbb{R}$ is a given force density and

$$
\mathbf{F}(t, \mathbf{y}):=\left(-2 \mu \mathbb{D}_{\mathbf{y}} \mathbf{u}(t, \mathbf{y})-[\mathbb{T}(\psi)](t, \mathbf{y})+p(t, \mathbf{y}) \mathbb{I}\right) \boldsymbol{v}_{\eta(t)} \circ \boldsymbol{\varphi}_{\eta(t)}\left|\operatorname{det} D \boldsymbol{\varphi}_{\eta(t)}\right| .
$$

Here, the tensor

$$
\mathbb{D}_{\mathbf{y}} \mathbf{u}=\frac{1}{2}\left(\nabla_{\mathbf{y}} \mathbf{u}+\nabla_{\mathbf{y}}^{T} \mathbf{u}\right)
$$

is the symmetric gradient of the fluid's velocity field. The elastic stress tensor $\mathbb{T}(\psi)$ will be introduced in the next subsection.

\subsection{Polymer Fluid}

A common mathematical model to describe the behaviour of complex fluids are the FENE-type models. For these models, the polymer molecules are idealized as a chain of beads and springs with prescribed finitely extensible nonlinear elastic (FENE) type spring potentials. For a finite but arbitrary natural number $K>1, K+1$ beads are connected by $K$ springs to form a polymer chain. This polymer is represented by a vector of $K$-finite vectors $\mathbf{q}=\left(\mathbf{q}_{1}^{T}, \ldots, \mathbf{q}_{K}^{T}\right)^{T} \in B$, where $B=\bigotimes_{i=1}^{K} B_{i} \subset \mathbb{R}^{3 K}$ is the Cartesian product of convex open sets $B_{i} \subset \mathbb{R}^{3}$ such that $\mathbf{q}_{i} \in B_{i}$ if and only if $-\mathbf{q}_{i} \in B_{i}$, see Fig. 2. On the mesoscopic level, we describe the evolutionary changes in the distribution of the bead-spring chain configuration by the FokkerPlanck equation for the polymer density function $\psi=\psi(t, \mathbf{x}, \mathbf{q})$ (depending on time $t \geq 0$, spatial position $\mathbf{x} \in \mathbb{R}^{3}$ and the prolongation vector $\mathbf{q} \in B$ of the polymer chain). On the macroscopic level, we consider a viscous fluid described by the incompressible Navier-Stokes equations for the fluid velocity $\mathbf{u}=\mathbf{u}(t, \mathbf{x})$ and pressure $p=p(t, \mathbf{x})$. The beads, which model the monomers that are joined by springs to form a polymer chain, unsettle the flow field around the chain once immersed in the fluid. These 
mesoscopic effects of the polymer molecules on the fluid motion are described by an elastic stress tensor $\mathbb{T}$. It is meant to describe the random movements of polymer chains/springs and can be modelled by prescribing spring potentials $U_{i}, i=1, \ldots, K$ for each of the $K$ springs. Here, for each $i=1, \ldots, K$, the potential $U_{i}$ is continuous on an interval $I_{i} \subset[0, \infty)$ containing the point 0 where $I_{i}$ is the image of $B_{i}$ under the mapping $\mathbf{q}_{i} \in B_{i} \mapsto \frac{1}{2}\left|\mathbf{q}_{i}\right|^{2}$. To be precise, we consider $U_{i} \in C_{\text {loc }}^{0,1}\left(I_{i} ;[0, \infty)\right)$ for each $i=1, \ldots, K$. Typically, these potentials will be such that $U_{i}(0)=0$ and also, be monotonically increasing and unbounded on the interval $I_{i}$, for each $i=1, \ldots, K$. The elastic spring force $\mathbf{F}_{i}: B_{i} \subset \mathbb{R}^{3} \rightarrow \mathbb{R}^{3}$ of the $i$ th spring and the associated Maxwellian $M_{i}$ are defined by

$$
\mathbf{F}_{i}\left(\mathbf{q}_{i}\right)=\nabla_{\mathbf{q}_{i}} U_{i}\left(\frac{1}{2}\left|\mathbf{q}_{i}\right|^{2}\right)=U_{i}^{\prime}\left(\frac{1}{2}\left|\mathbf{q}_{i}\right|^{2}\right) \mathbf{q}_{i}, \quad i=1, \ldots, K
$$

and

$$
M_{i}\left(\mathbf{q}_{i}\right)=\frac{e^{-U_{i}\left(\frac{1}{2}\left|\mathbf{q}_{i}\right|^{2}\right)}}{\int_{B_{i}} e^{-U_{i}\left(\frac{1}{2}\left|\mathbf{q}_{i}\right|^{2}\right)} \mathrm{d} \mathbf{q}_{i}}, \quad i=1, \ldots, K
$$

respectively, such that $\int_{B_{i}} M_{i}\left(\mathbf{q}_{i}\right) \mathrm{d} \mathbf{q}_{i}=1$ for each $i=1, \ldots, K$. The (total) Maxwellian is then given by

$$
M(\mathbf{q}):=\prod_{i=1}^{K} M_{i}\left(\mathbf{q}_{i}\right), \quad \mathbf{q}=\left(\mathbf{q}_{1}^{T}, \ldots, \mathbf{q}_{K}^{T}\right)^{T} \in B=\bigotimes_{i=1}^{K} B_{i}
$$

and also satisfies $\int_{B} M(\mathbf{q}) \mathrm{d} \mathbf{q}=1$. We observe from (1.12)-(1.14) that

$$
M(\mathbf{q}) \nabla_{\mathbf{q}_{i}} \frac{1}{M(\mathbf{q})}=\frac{-1}{M(\mathbf{q})} \nabla_{\mathbf{q}_{i}} M(\mathbf{q})=\mathbf{F}_{i}\left(\mathbf{q}_{i}\right)
$$

for each $i=1, \ldots, K$ (Fig. 2).

Before we continue, we now give some examples of the precise force-laws (1.12) used in the literature, see Bird et al. (1987, Table 11.5-1). For simplicity of the presentation, we only describe the 'dumbbell' models corresponding to $K=1$.

Example 1.1 (Hookean dumbbell model) This model has a prescribed linear spring force and (equivalently) linear spring potential given by $\mathbf{F}(\mathbf{q})=\mathbf{q}$ where $\mathbf{q} \in B=\mathbb{R}^{d}$, $d=2,3$ and $U(s)=s, s \in \mathbb{R}_{\geq 0}$ respectively. Therefore, the beads coalesce at the origin $\mathbf{0} \in B$ when the spring force is zero. The main drawback of this model is that it admits arbitrarily large spring extension making it physically unrealistic.

Example 1.2 ('Linear-locked' Tanner's dumbbell model) This model is a realistic variant of the Hookean dumbbell model with the same linear force law and linear spring potential except that now, $\mathbf{q} \in B=B(\mathbf{0}, \sqrt{b}) \subset \mathbb{R}^{d}, d=2,3$ where $B=B(\mathbf{0}, \sqrt{b})$ is a bounded open set centred at $\mathbf{0} \in \mathbb{R}^{d}$ of radius $\sqrt{b}$, with $b>0$. The prescribed radius $\sqrt{b}>0$ thus denotes the extent to which the springs can be stretched. 


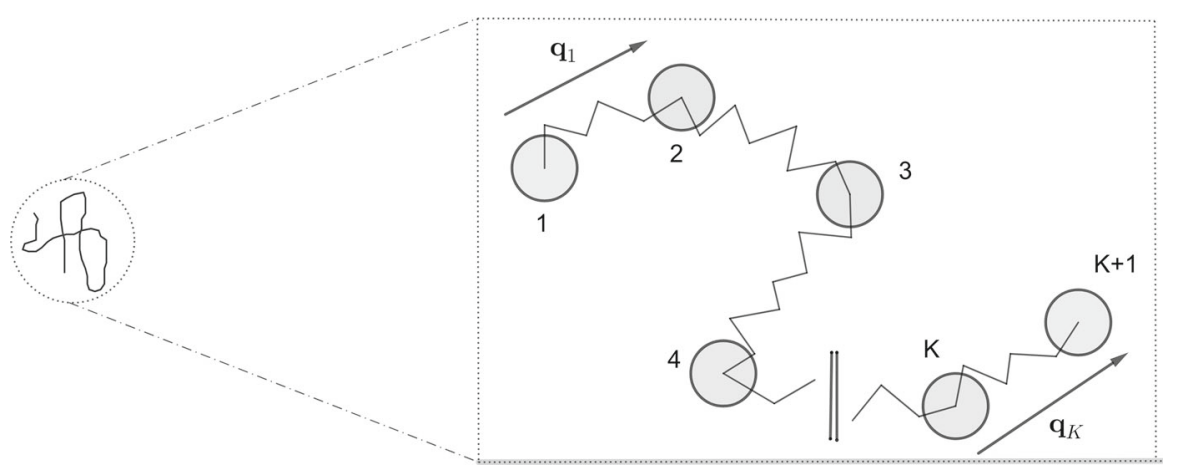

Fig. 2 We model a polymer (circled on the left) as a bead-spring chain consisting of $K+1$ beads connected by $K$ springs (boxed on the right)

Example 1.3 (FENE [finitely extensible nonlinear elastic] dumbbell model) This model also corresponds to the case $K=1$ but with a nonlinear spring force given by $\mathbf{F}(\mathbf{q})=\left(1-|\mathbf{q}|^{2} / b\right)^{-1} \mathbf{q}$ where $\mathbf{q} \in B=B(\mathbf{0}, \sqrt{b}) \subset \mathbb{R}^{d}, d=2,3$. Equivalently, the nonlinear spring potential is given $U(s)=-\frac{b}{2} \log \left(1-\frac{2 s}{b}\right), s \in\left[0, \frac{b}{2}\right)$.

With respect to regularity, we assume that the Maxwellian satisfies the following conditions:

$$
M \in C(\bar{B}) \cap C_{\mathrm{loc}}^{0,1}(B) \cap W_{0}^{1,1}(B), \quad M \geq 0, \quad M^{-1} \in C_{\mathrm{loc}}(B) .
$$

Now, for a given probability density function $\psi=\psi(t, \mathbf{x}, \mathbf{q})$ of a polymer, we let

$$
\Xi(t, x)=\int_{B} \psi(t, \mathbf{x}, \mathbf{q}) \mathrm{d} \mathbf{q}, \quad(t, \mathbf{x}) \in I \times \Omega_{\eta(t)}
$$

be the polymer number density. The elastic stress tensor $\mathbb{T}$ is then given by

$$
\mathbb{T}(\psi)=k \sum_{i=1}^{K} \mathbb{T}_{i}(\psi)-k(K+1) \Xi \mathbb{I}-\partial \Xi^{2} \mathbb{I}
$$

where $\mathbb{I}$ is the identity matrix, $k>0$ and $\precsim \geq 0$ are constants and for each $i=1, \ldots, K$,

$$
\begin{aligned}
\mathbb{T}_{i}(\psi) & =\int_{B} \psi(t, \mathbf{x}, \mathbf{q}) U_{i}^{\prime}\left(\frac{1}{2}\left|\mathbf{q}_{i}\right|^{2}\right) \mathbf{q}_{i} \otimes \mathbf{q}_{i} \mathrm{~d} \mathbf{q} \\
& =\int_{B} \psi(t, \mathbf{x}, \mathbf{q}) \mathbf{F}_{i}\left(\mathbf{q}_{i}\right) \otimes \mathbf{q}_{i} \mathrm{~d} \mathbf{q}
\end{aligned}
$$

elucidates how the polymers - described by the force law for the $i$ th spring-are transmitted through the fluid.

In addition to the elastic stress tensor $\mathbb{T}$, we consider an external volume force $\mathbf{f}:(t, \mathbf{x}) \in I \times \Omega_{\eta(t)} \mapsto \mathbf{f}(t, \mathbf{x}) \in \mathbb{R}^{3}$ in the fluid motion. This force may account for 
the influence of gravity and/or electric force as well as artificial forces produced, for example, by an ultracentrifuge.

The coupled system is now described by the incompressible Navier-StokesFokker-Planck system in the moving domain $I \times \Omega_{\eta(t)}$ for a given function $\eta$ : $I \times \partial \Omega \rightarrow(-L, L)$. We wish to find the fluid's velocity field $\mathbf{u}:(t, \mathbf{x}) \in I \times \Omega_{\eta(t)} \mapsto$ $\mathbf{u}(t, \mathbf{x}) \in \mathbb{R}^{3}$, the pressure $p:(t, \mathbf{x}) \in I \times \Omega_{\eta(t)} \mapsto p(t, \mathbf{x}) \in \mathbb{R}$ and the probability density function $\psi:(t, \mathbf{x}, \mathbf{q}) \in I \times \Omega_{\eta(t)} \times B \mapsto \psi(t, \mathbf{x}, \mathbf{q}) \in[0, \infty)$ such that the equations

$$
\begin{aligned}
& \operatorname{div}_{\mathbf{x}} \mathbf{u}=0, \\
& \partial_{t} \mathbf{u}+\left(\mathbf{u} \cdot \nabla_{\mathbf{x}}\right) \mathbf{u}+\nabla_{\mathbf{x}} p=\mu \Delta_{\mathbf{x}} \mathbf{u}+\operatorname{div}_{\mathbf{x}} \mathbb{T}(\psi)+\mathbf{f}, \\
& \partial_{t} \psi+\left(\mathbf{u} \cdot \nabla_{\mathbf{x}}\right) \psi=\varepsilon \Delta_{\mathbf{x}} \psi-\sum_{i=1}^{K} \operatorname{div}_{\mathbf{q}_{i}}\left(\left(\nabla_{\mathbf{x}} \mathbf{u}\right) \mathbf{q}_{i} \psi\right) \\
& +\frac{1}{4 \lambda} \sum_{i=1}^{K} \sum_{j=1}^{K} A_{i j} \operatorname{div}_{\mathbf{q}_{i}}\left(M \nabla_{\mathbf{q}_{j}} \frac{\psi}{M}\right)
\end{aligned}
$$

are satisfied weakly in $I \times \Omega_{\eta(t)} \times B$ subject to the following initial $(t=0)$ condition and boundary $\left(\mathbf{y} \in \partial \Omega_{\eta(t)}\right.$ or $\left.\mathbf{q}_{i} \in \partial \bar{B}_{i}\right)$ conditions for $\mathrm{i}=1, \ldots, \mathrm{K}$,

$$
\begin{aligned}
& {\left[\frac{1}{4 \lambda} \sum_{j=1}^{K} A_{i j} M \nabla_{\mathbf{q}_{j}} \frac{\psi}{M}-\left(\nabla_{\mathbf{x}} \mathbf{u}\right) \mathbf{q}_{i} \psi\right] \cdot \mathbf{n}_{i}=0 \text { on } I \times \Omega_{\eta(t)} \times \partial \bar{B}_{i},} \\
& \varepsilon \nabla_{\mathbf{y}} \psi \cdot \boldsymbol{v}_{\eta(t)}=0 \quad \text { on } I \times \partial \Omega_{\eta(t)} \times B, \\
& \mathbf{u}(0, \cdot)=\mathbf{u}_{0} \quad \text { in } \Omega_{\eta_{0}}, \\
& \psi(0, \cdot, \cdot)=\psi_{0} \geq 0 \quad \text { in } \Omega_{\eta_{0}} \times B .
\end{aligned}
$$

The parameter $\mu>0$ is the viscosity coefficient, $\varepsilon>0$ is the center-of-mass diffusion coefficient, $\lambda>0$ is the Deborah number De, the $A_{i j}$ 's are the components of the symmetric positive definite Rouse matrix $\left(A_{i j}\right)_{i, j=1}^{K}$ whose smallest eigenvalue is $A_{0}>0$ and $\mathbf{n}_{i}$ is a unit outward normal vector to $\partial B_{i}$.

If we now return to (1.17) for a moment, we observe that by formally integrating (1.22) over the open set $B$ and using the boundary condition (1.23), then $\Xi$ satisfies the following viscous transport equation

$$
\partial_{t} \Xi+\left(\mathbf{u} \cdot \nabla_{\mathbf{x}}\right) \Xi=\varepsilon \Delta_{\mathbf{x}} \Xi
$$

weakly in $I \times \Omega_{\eta(t)}$ subject to the following initial and boundary conditions

$$
\begin{array}{lr}
\varepsilon \nabla_{\mathbf{y}} \Xi \cdot \boldsymbol{v}_{\eta(t)}=0 & \text { on } I \times \partial \Omega_{\eta(t)}, \\
\Xi_{0}=\int_{B} \psi_{0}(\cdot, \mathbf{q}) \mathrm{d} \mathbf{q} \geq 0 & \text { in } \Omega_{\eta_{0}} .
\end{array}
$$


The structure of the tensor $\mathbb{T}$, given by (1.18), means that the analysis of (1.27)-(1.29) is essential to the analysis of the extra stress tensor $\mathbb{T}$.

If we now define $\widehat{\psi}:=\psi / M$, then the full extra stress tensor (1.18) may be rewritten as

$$
\mathbb{T}(M \widehat{\psi})=\mathbb{T}(\psi)=k \sum_{i=1}^{K} \int_{B} M(\mathbf{q}) \nabla_{\mathbf{q}_{i}} \widehat{\psi}(t, \mathbf{x}, \mathbf{q}) \otimes \mathbf{q}_{i} \mathrm{~d} \mathbf{q}-k \Xi \mathbb{I}-\partial \Xi^{2} \mathbb{I}
$$

The Fokker-Planck equation (1.22) then becomes

$$
\begin{aligned}
\partial_{t}(M \widehat{\psi})+\left(\mathbf{u} \cdot \nabla_{\mathbf{x}}\right) M \widehat{\psi}= & \varepsilon \Delta_{\mathbf{x}}(M \widehat{\psi})-\sum_{i=1}^{K} \operatorname{div}_{\mathbf{q}_{i}}\left(M\left(\nabla_{\mathbf{x}} \mathbf{u}\right) \mathbf{q}_{i} \widehat{\psi}\right) \\
& +\frac{1}{4 \lambda} \sum_{i=1}^{K} \sum_{j=1}^{K} A_{i j} \operatorname{div}_{\mathbf{q}_{i}}\left(M \nabla_{\mathbf{q}_{j}} \widehat{\psi}\right)
\end{aligned}
$$

subject to the initial and boundary conditions for $i=1, \ldots, K$,

$$
\begin{aligned}
& {\left[\frac{1}{4 \lambda} \sum_{j=1}^{K} A_{i j} M \nabla_{\mathbf{q}_{j}} \widehat{\psi}-M\left(\nabla_{\mathbf{x}} \mathbf{u}\right) \mathbf{q}_{i} \widehat{\psi}\right] \cdot \mathbf{n}_{i}=0 \quad \text { on } I \times \Omega_{\eta(t)} \times \partial \bar{B}_{i},} \\
& \varepsilon \nabla_{\mathbf{y}} \widehat{\psi} \cdot \boldsymbol{v}_{\eta(t)}=0 \quad \text { on } I \times \partial \Omega_{\eta(t)} \times B, \\
& \widehat{\psi}(0, \cdot, \cdot)=\widehat{\psi}_{0} \geq 0 \quad \text { in } \Omega_{\eta_{0}} \times B .
\end{aligned}
$$

\subsection{Plan of the Paper}

In the following, we give the outline of the rest of this paper. As stated in the abstract above, we aim to show the existence of a weak solution to the coupled system (1.20)(1.21), (1.30)-(1.34) and (1.7)-(1.9) where the solution exists globally in time until the shell approaches a self-intersection or the $W^{2,2}$-coercivity of the Koiter energy given in (1.5) degenerates. Therefore, after collecting some preliminary notations and concepts in Sects. 2.1 and 2.2, we make the exact notion of a solution to our system precise in Sect. 2.3. We also state our main theorem, Theorem 2.6 in Sect. 2.4. We then move to Sect. 3, where we show how to formally derive a priori estimates and also, introduce the energy and relative entropy of our system.

Our principal strategy to solve the coupled system consists in regularising the shell (and the convective terms) and to decouple the fluid-structure problem from the Fokker-Planck equation. For this reason, in Sect. 4, we solve the Fokker-Planck equation in a variable (but given) domain for a given (and smooth) velocity field. On the other hand, for a given probability density function, we solve the fluid-structure problem by following the fixed-point arguments in Lengeler and Růžička (2014). This is done in Sect. 5. Finally, we pass to the limit in the regularisation layer in Sect. 6. For this, we are required to rigorously prove the entropy estimates from Sect. 3 (see 
Remark (2.5) for the notion of an entropy estimate) and to apply compactness methods to pass to the limit in the nonlinear terms of our system.

\section{Preliminaries and Main Result}

In this section, we fix the notation, collect some preliminary material on function spaces and present the main result.

\subsection{Notations}

The following quantities: $t \in \bar{I}$, representing the time variable, $\mathbf{x} \in \Omega$, representing the spatial variable, and $\mathbf{q} \in B$, representing the elongation vector of a polymer molecule, will denote the independent variables we shall use throughout this work. The domain $B=\bigotimes_{i=1}^{K} B_{i} \subset \mathbb{R}^{3 K}$ is the Cartesian product of $K$ convex and bounded open sets $B_{i} \subset \mathbb{R}^{3}$ for which $\mathbf{q}_{i} \in B_{i}$ if and only if $-\mathbf{q}_{i} \in B_{i}$. In particular, the origin $\mathbf{0} \in \mathbb{R}^{3}$ is contained in each set $B_{i}$ whose boundary we denote by $\partial B_{i}$. We further have

$$
\partial B=\bigcup_{i=1}^{K} \partial \overline{B_{i}}, \quad \text { where } \partial \overline{B_{i}}:=B_{1} \times \ldots \times B_{i-1} \times \partial B_{i} \times B_{i+1} \times \ldots \times B_{K}
$$

with $\mathbf{n}_{i}$ being a unit outward normal vector to $\partial B_{i}, i=1, \ldots, K$. For functions $F$ and $G$ and a variable $p$, we write $F \lesssim G$ and $F \lesssim_{p} G$ if there exists a generic constant $c>0$ and another such constant $c(p)>0$ which now depends on $p$ such that $F \leq c G$ and $F \leq c(p) G$ holds respectively. The symbol $|\cdot|$ may be used in four different context. For a scaler function $f \in \mathbb{R},|f|$ denotes the absolute value of $f$. For a vector $\mathbf{f} \in \mathbb{R}^{n}$ where $n>1$ is an integer, $|\mathbf{f}|$ denotes the Euclidean norm of $\mathbf{f}$. For a square matrix $\mathbb{F} \in \mathbb{R}^{n \times n}$ where $n>1$ is an integer, $|\mathbb{F}|$ shall denote the Frobenius norm $\sqrt{\operatorname{trace}\left(\mathbb{F}^{T} \mathbb{F}\right)}$. Finally, if $S \subset \mathbb{R}^{n}$ is a (sub)set, then $|S|$ is the $n$-dimensional Lebesgue measure of $S$.

Let $\mathcal{O} \subset \mathbb{R}^{d}$ be a measureable set. By $L^{p}(\mathcal{O})\left[\right.$ respectively $\left.L^{p}\left(\mathcal{O} ; \mathbb{R}^{3}\right)\right], W^{s, p}(\mathcal{O})$ [respectively $W^{s, p}\left(\mathcal{O} ; \mathbb{R}^{3}\right)$ ] and $D^{s, p}(\mathcal{O})$ [respectively $D^{s, p}\left(\mathcal{O} ; \mathbb{R}^{3}\right)$ ] for $1 \leq p \leq \infty$ and $s \in \mathbb{N}$, we denote respectively, the standard Lebesgue spaces, Sobolev spaces, homogeneous Sobolev spaces for scalar-valued [respectively $\mathbb{R}^{3}$-vector-valued] functions defined on $\mathcal{O}$. The dual space of $W^{s, p}(\mathcal{O})$ will be denoted by $W^{-s, p}(\mathcal{O})$. By $L_{\operatorname{div}_{\mathbf{x}}}^{p}\left(\mathcal{O} ; \mathbb{R}^{3}\right)$, we mean vector-valued measurable functions $\mathbf{v}: \mathcal{O} \rightarrow \mathbb{R}^{3}$ such that $\operatorname{div}_{\mathbf{x}} \mathbf{v}=0$ in the distributional sense and $\|\mathbf{v}\|_{L^{p}(\mathcal{O})}<\infty$. The Sobolev space $W_{0}^{s, p}(\mathcal{O})$ is endowed with zero boundary condition (i.e. it is the closure of the smooth and compactly supported functions in $\left.W^{s, p}(\mathcal{O})\right)$. Also, for $s \in(0,1)$ and $1 \leq p<\infty$, we define the fractional Sobolev space $W^{s, p}(\mathcal{O})$ as the set of all measurable functions $f: \Omega \rightarrow \mathbb{R}$ such that

$$
\|f\|_{W^{s, p}(\mathcal{O})}^{p}=\int_{\mathcal{O}}|f(\mathbf{x})|^{p} \mathrm{~d} \mathbf{x}+\int_{\mathcal{O}} \int_{\mathcal{O}} \frac{|f(\mathbf{x})-f(\mathbf{z})|^{p}}{|\mathbf{x}-\mathbf{z}|^{d+p s}} \mathrm{~d} \mathbf{x} \mathrm{d} \mathbf{z}<\infty .
$$


In general, for a separable Banach space $\left(X,\|\cdot\|_{X}\right)$, we denote by $L^{p}(0, T ; X)$, the space of Bochner-measurable functions $u:(0, T) \rightarrow X$ such that $\|u\|_{X} \in L^{p}(0, T)$. Similarly, we consider the space $L^{p}(\mathcal{O} ; X)$ for a measurable set $\mathcal{O} \subset \mathbb{R}^{d}$. Also, $C(\mathcal{O} ; X)$ is the set of continuous functions $u: \mathcal{O} \rightarrow X$. Finally, for any nonnegative $N \in C(\mathcal{O})$, where $\mathcal{O} \subset \mathbb{R}^{d}$ is a measurable set, and for a constant $p \geq 1$, we denote by

$$
\begin{aligned}
& L_{N}^{p}(\mathcal{O})=\left\{f \in L_{\mathrm{loc}}^{p}(\mathcal{O}):\|f\|_{L_{N}^{p}(\mathcal{O})}^{p}<\infty\right\}, \\
& \quad W_{N}^{1, p}(\mathcal{O})=\left\{f \in W_{\mathrm{loc}}^{1, p}(\mathcal{O}):\|f\|_{W_{N}^{1, p}(\mathcal{O})}^{p}<\infty\right\},
\end{aligned}
$$

the weighted $L^{p}$ and $W^{1, p}$ spaces over $\mathcal{O}$ with norms

$$
\begin{aligned}
\|f\|_{L_{N}^{p}(\mathcal{O})}^{p} & :=\int_{\mathcal{O}} N(\mathbf{z})|f(\mathbf{z})|^{p} \mathrm{~d} \mathbf{z} \\
\|f\|_{W_{N}^{1, p}(\mathcal{O})}^{p} & :=\int_{\mathcal{O}} N(\mathbf{z})\left(|f(\mathbf{z})|^{p}+\left|\nabla_{\mathbf{z}} f(\mathbf{z})\right|^{p}\right) \mathrm{d} \mathbf{z},
\end{aligned}
$$

respectively.

\subsection{Function Spaces on Variable Domains}

The spatial domain $\Omega$ is a nonempty bounded subset of $\mathbb{R}^{3}$ with smooth boundary and an outer unit normal $\boldsymbol{v}, \partial \Omega$ is the shell of $\Omega \subset \mathbb{R}^{3}$. We use $\mathbf{y} \in \partial \Omega$ to emphasis spatial boundary points with a corresponding surface measure dy. To further clarify, we identify the shell as a usual boundary by tracing out its 2-dimensional mid-section $\omega \in \mathbb{R}^{2}$, see Fig 1 . In the following, $\bar{I}$ is the closure of $I=(0, T)$, a time interval where $T>0$ is a constant. For $\eta \in C(\bar{I} \times \partial \Omega)$ satisfying $\|\eta\|_{L^{\infty}(I \times \partial \Omega)}<L$, we shall abuse notation and denote the deformed spacetime cylinder $\cup_{t \in I}\{t\} \times \Omega_{\eta(t)} \subset \mathbb{R}^{4}$ by either $\Omega_{\eta(t)}^{I}$ or $I \times \Omega_{\eta(t)}$. We are now in the position to define function spaces on a variable domain.

Definition 2.1 (Function spaces) We define for $1 \leq p, r \leq \infty$,

$$
\begin{aligned}
L^{p}\left(I ; L^{r}\left(\Omega_{\eta(t)}\right)\right):= & \left\{v \in L^{1}\left(I \times \Omega_{\eta(t)}\right): v(t, \cdot) \in L^{r}\left(\Omega_{\eta(t)}\right)\right. \\
& \text { for a.e. } t,\|v(t, \cdot)\|_{\left.L^{r}\left(\Omega_{\eta(t)}\right) \in L^{p}(I)\right\},} \\
L^{p}\left(I ; W^{1, r}\left(\Omega_{\eta(t)}\right)\right):= & \left\{v \in L^{p}\left(I ; L^{r}\left(\Omega_{\eta(t)}\right)\right): \nabla_{\mathbf{x}} v \in L^{p}\left(I ; L^{r}\left(\Omega_{\eta(t)}\right)\right)\right\} .
\end{aligned}
$$

We now give a concept of convergence in variable domains which is similar to Breit and Schwarzacher (2018, Sec. 2.3).

Definition 2.2 (Convergence) Let $\left(\eta_{i}\right) \subset C(\bar{I} \times \partial \Omega ;[-\theta L, \theta L]), \theta \in(0,1)$ be a sequence such that $\eta_{i} \rightarrow \eta$ uniformly in $\bar{I} \times \omega$. Let $p_{1}, p_{2}, p_{3} \in[1, \infty]$ and let $M$ satisfy (1.16) or be identically equal to one. Then; 
(a) we say that a sequence $g_{i} \in L^{p_{1}}\left(I ; L^{p_{2}}\left(\Omega_{\eta_{i}(t)} ; L_{M}^{p_{3}}(B)\right)\right)$ converges strongly to $g$ in $L^{p_{1}}\left(I ; L^{p_{2}}\left(\Omega_{\eta_{i}(t)} ; L_{M}^{p_{3}}(B)\right)\right)$ with respect to $\eta_{i}$, denoted by $g_{i} \rightarrow^{\eta} g$ in $L^{p_{1}}\left(I ; L^{p_{2}}\left(\Omega_{\eta_{i}(t)} ; L_{M}^{p_{3}}(B)\right)\right)$, if

$$
\chi_{\Omega_{\eta_{i}(t)}} g_{i} \rightarrow \chi_{\Omega_{\eta(t)}} g \quad \text { in } \quad L^{p_{1}}\left(I ; L^{p_{2}}\left(\mathbb{R}^{3} ; L_{M}^{p_{3}}(B)\right)\right)
$$

(b) for $p_{1}, p_{2}, p_{3} \in[1, \infty)$, we say that a sequence $g_{i} \in L^{p_{1}}\left(I ; L^{p_{2}}\left(\Omega_{\eta_{i}(t)} ; L_{M}^{p_{3}}(B)\right)\right)$ converges weakly to $g$ in $L^{p_{1}}\left(I ; L^{p_{2}}\left(\Omega_{\eta(t)} ; L_{M}^{p_{3}}(B)\right)\right)$ with respect to $\eta_{i}$, denoted by $g \rightarrow \eta g$ in $L^{p_{1}}\left(I ; L^{p_{2}}\left(\Omega_{\eta_{i}(t)} ; L_{M}^{p_{3}}(B)\right)\right)$, if

$$
\chi_{\Omega_{\eta_{i}(t)}} g_{i} \rightarrow \chi_{\Omega_{\eta(t)}} g \text { in } \quad L^{p_{1}}\left(I ; L^{p_{2}}\left(\mathbb{R}^{3} ; L_{M}^{p_{3}}(B)\right)\right)
$$

(c) for $p_{1}=\infty$ and $p_{2}, p_{3} \in[1, \infty)$, we say that a sequence $g_{i} \in L^{\infty}\left(I ; L^{p_{2}}\left(\Omega_{\eta_{i}(t)}\right.\right.$; $\left.\left.L_{M}^{p_{3}}(B)\right)\right)$ converges weakly* to $g$ in $L^{\infty}\left(I ; L^{p_{2}}\left(\Omega_{\eta(t)} ; L_{M}^{p_{3}}(B)\right)\right)$ with respect to $\eta_{i}$, denoted by $g_{i} \rightarrow^{*, \eta} g$ in $L^{\infty}\left(I ; L^{p_{2}}\left(\Omega_{\eta_{i}(t)} ; L_{M}^{p_{3}}(B)\right)\right)$, if

$$
\chi_{\Omega_{\eta_{i}(t)}} g_{i} \rightarrow^{*} \chi_{\Omega_{\eta(t)}} g \text { in } \quad L^{\infty}\left(I ; L^{p_{2}}\left(\mathbb{R}^{3} ; L_{M}^{p_{3}}(B)\right)\right)
$$

Definition 2.2 can be extended in a canonical way to Sobolev spaces.

Since we are dealing with boundary value problems, we need a concept of traces on variable domains. The following lemma is a modification of Lengeler and Růžička (2014, Corollary 2.9), see also (Muha 2014). We recall the transform $\varphi_{\eta}$ from Sect. 1.2.

Lemma 2.3 (Trace operator) Let $1<p<3$ and $\eta \in W^{2,2}(\partial \Omega)$ with $\|\eta\|_{L^{\infty}(\partial \Omega)}<L$. Then the linear mapping $\operatorname{tr}_{\eta}:\left.v \mapsto v \circ \varphi_{\eta}\right|_{\partial \Omega}$ is well defined and continuous from $W^{1, p}\left(\Omega_{\eta}\right)$ to $W^{1-\frac{1}{r}, r}(\partial \Omega)$ for all $r \in(1, p)$ and well defined and continuous from $W^{1, p}\left(\Omega_{\eta}\right)$ to $L^{q}(\partial \Omega)$ for all $1<q<\frac{2 p}{3-p}$. The continuity constants depend only on $\Omega, p$, and $\|\eta\|_{W^{2,2}(\partial \Omega)}$.

\subsection{Concept of a Solution}

In order to describe the notion of a solution that we wish to construct, we first define the following energy functionals:

$$
\begin{aligned}
\mathcal{E}(t):= & \|\Xi(t, \mathbf{x})\|_{L^{\infty}\left(\Omega_{\eta(t)}\right)}^{2}+\int_{\Omega_{\eta(t)}} \frac{1}{2}|\mathbf{u}(t, \mathbf{x})|^{2} \mathrm{~d} \mathbf{x}+\int_{\omega} \frac{1}{2}\left|\partial_{t} \eta(t, \mathbf{y})\right|^{2} \mathrm{~d} \mathbf{y}+K(\eta(t)) \\
& +k \int_{\Omega_{\eta(t)} \times B} M(\mathbf{q}) \mathcal{F}(\widehat{\psi}(t, \mathbf{x}, \mathbf{q})) \mathrm{d} \mathbf{q} \mathrm{d} \mathbf{x},
\end{aligned}
$$

where

$$
\mathcal{F}(s):=s \ln s+\mathrm{e}^{-1} \quad \text { for } \quad s \geq 0
$$


is the entropy function that generates the physical relative (with respect to the Maxwellian) entropy

$$
\mathcal{E}_{\mathcal{F}}^{\eta(t)}(\widehat{\psi} \mid M):=\int_{\Omega_{\eta(t)} \times B} M \mathcal{F}(\widehat{\psi}) \mathrm{d} \mathbf{q} \mathrm{d} \mathbf{x}
$$

and let

$$
\begin{aligned}
\mathcal{E}(0) & :=\left\|\Xi_{0}(\mathbf{x})\right\|_{L^{\infty}\left(\Omega_{\eta(0)}\right)}^{2}+\int_{\Omega_{\eta_{0}}} \frac{1}{2}\left|\mathbf{u}_{0}(\mathbf{x})\right|^{2} \mathrm{~d} \mathbf{x}+\int_{\omega} \frac{1}{2}\left|\eta_{1}(\mathbf{y})\right|^{2} \mathrm{~d} \mathbf{y}+K\left(\eta_{0}\right) \\
& +k \int_{\Omega_{\eta_{0}} \times B} M \mathcal{F}\left(\widehat{\psi}_{0}(\mathbf{x}, \mathbf{q})\right) \mathrm{d} \mathbf{q} \mathrm{d} \mathbf{x}
\end{aligned}
$$

be the initial energy, recall (1.8). With the above information in hand, we now proceed to make rigorous, what we mean by a solution.

Definition 2.4 (Finite energy weak solution) Let (f), $\left.g, \eta_{0}, \widehat{\psi}_{0}, \mathbf{u}_{0}, \eta_{1}\right)$ be a dataset such that

$$
\begin{aligned}
& \mathbf{f} \in L^{2}\left(I ; L_{\mathrm{loc}}^{2}\left(\mathbb{R}^{3} ; \mathbb{R}^{3}\right)\right), \quad g \in L^{2}\left(I ; L^{2}(\omega)\right), \quad \eta_{0} \in W^{2,2}(\omega) \text { with }\left\|\eta_{0}\right\|_{L^{\infty}(\omega)}<L \\
& \quad 0 \leq \widehat{\psi}_{0} \in L^{2}\left(\Omega_{\eta_{0}} ; L_{M}^{2}(B)\right), \quad \mathbf{u}_{0} \in L_{\operatorname{div}_{\mathbf{x}}}^{2}\left(\Omega_{\eta_{0}} ; \mathbb{R}^{3}\right) \text { is such that } \\
& \quad \operatorname{tr}_{\eta_{0}} \mathbf{u}_{0}=\eta_{1} \gamma\left(\eta_{0}\right), \quad \eta_{1} \in L^{2}(\omega) .
\end{aligned}
$$

In addition, we assume

$$
\Xi_{0} \in L^{\infty}\left(\Omega_{\eta_{0}}\right) \text { where } \Xi_{0}=\int_{B} M(\mathbf{q}) \widehat{\psi}_{0}(\cdot, \mathbf{q}) \text { d } \mathbf{q} \text { in } \Omega_{\eta_{0}} .
$$

We call the triple $(\mathbf{u}, \widehat{\psi}, \eta)$ a finite energy weak solution to the system (1.20)-(1.21), (1.30)-(1.34) and (1.7)-(1.9) with data (f, $\left.g, \eta_{0}, \widehat{\psi}_{0}, \mathbf{u}_{0}, \eta_{1}\right)$ provided that the following holds:

(a) the velocity $\mathbf{u}$ satisfies

$$
\mathbf{u} \in L^{\infty}\left(I ; L^{2}\left(\Omega_{\eta(t)} ; \mathbb{R}^{3}\right)\right) \cap L^{2}\left(I ; W_{\operatorname{div}_{\mathbf{x}}}^{1,2}\left(\Omega_{\eta(t)} ; \mathbb{R}^{3}\right)\right) \text { with } \mathbf{u}\left(t, \boldsymbol{\varphi}_{\eta}(t, \mathbf{y})\right)=\partial_{t} \eta(t, \mathbf{y}) \boldsymbol{v}(\mathbf{y})
$$

in the sense of traces and $\eta$ satisfies

$$
\eta \in W^{1, \infty}\left(I ; L^{2}(\omega)\right) \cap L^{\infty}\left(I ; W^{2,2}(\omega)\right) \text { with }\|\eta\|_{L^{\infty}(I \times \omega)}<L
$$

and for all $(\phi, \varphi) \in C^{\infty}(\bar{I} \times \omega) \times C^{\infty}\left(\bar{I} \times \mathbb{R}^{3} ; \mathbb{R}^{3}\right)$ with $\phi(T, \cdot)=0, \varphi(T, \cdot)=0$, $\operatorname{div}_{\mathbf{x}} \varphi=0$ and $\operatorname{tr}_{\eta} \varphi=\phi \boldsymbol{v}$, we have 


$$
\begin{aligned}
\int_{I} & \frac{\mathrm{d}}{\mathrm{d} t}\left(\int_{\Omega_{\eta(t)}} \mathbf{u} \cdot \boldsymbol{\varphi} \mathrm{d} \mathbf{x}+\int_{\omega} \partial_{t} \eta \phi \mathrm{d} \mathbf{y}\right) \mathrm{d} t \\
= & \int_{I} \int_{\Omega_{\eta(t)}}\left(\mathbf{u} \cdot \partial_{t} \boldsymbol{\varphi}+\mathbf{u} \otimes \mathbf{u}: \nabla_{\mathbf{x}} \boldsymbol{\varphi}\right) \mathrm{d} \mathbf{x} \mathrm{d} t \\
& -\int_{I} \int_{\Omega_{\eta(t)}}\left(\mu \nabla_{\mathbf{x}} \mathbf{u}: \nabla_{\mathbf{x}} \boldsymbol{\varphi}+\mathbb{T}(\psi): \nabla_{\mathbf{x}} \boldsymbol{\varphi}-\mathbf{f} \cdot \boldsymbol{\varphi}\right) \mathrm{d} \mathbf{x} \mathrm{d} t \\
& +\int_{I} \int_{\omega}\left(\partial_{t} \eta \partial_{t} \phi+g \phi\right) \mathrm{d} \mathbf{y} \mathrm{d} t-\int_{I}\left\langle K^{\prime}(\eta), \phi\right\rangle \mathrm{d} t ;
\end{aligned}
$$

(b) the probability density function $\widehat{\psi}$ satisfies:

$$
\begin{aligned}
& \widehat{\psi} \geq 0 \text { a.e. in } I \times \Omega_{\eta(t)} \times B, \\
& \widehat{\psi} \in L^{\infty}\left(I \times \Omega_{\eta(t)} ; L_{M}^{1}(B)\right), \\
& \mathcal{F}(\widehat{\psi}) \in L^{\infty}\left(I ; L^{1}\left(\Omega_{\eta(t)} ; L_{M}^{1}(B)\right)\right), \\
& \sqrt{\widehat{\psi}} \in L^{2}\left(I ; L^{2}\left(\Omega_{\eta(t)} ; W_{M}^{1,2}(B)\right)\right) \cap L^{2}\left(I ; D^{1,2}\left(\Omega_{\eta(t)} ; L_{M}^{2}(B)\right)\right), \\
& \Xi(t, \mathbf{x})=\int_{B} M \widehat{\psi}(t, \mathbf{x}, \mathbf{q}) \mathrm{d} \mathbf{q} \in L^{\infty}\left(I \times \Omega_{\eta(t)}\right) \cap L^{2}\left(I ; W^{1,2}\left(\Omega_{\eta(t)}\right)\right) ;
\end{aligned}
$$

and for all $\varphi \in C^{\infty}\left(\bar{I} \times \mathbb{R}^{3} \times \bar{B}\right)$, we have

$$
\begin{aligned}
& \int_{I} \frac{\mathrm{d}}{\mathrm{d} t} \int_{\Omega_{\eta(t)} \times B} M \widehat{\psi} \varphi \mathrm{d} \mathbf{q} \mathrm{d} \mathbf{x} \mathrm{d} t=\int_{I \times \Omega_{\eta(t)} \times B}\left(M \widehat{\psi} \partial_{t} \varphi+M \mathbf{u} \widehat{\psi} \cdot \nabla_{\mathbf{x}} \varphi-\varepsilon M \nabla_{\mathbf{x}} \widehat{\psi} \cdot \nabla_{\mathbf{x}} \varphi\right) \mathrm{d} \mathbf{q} \mathrm{d} \mathbf{x} \mathrm{d} t \\
& \quad+\sum_{i=1}^{K} \int_{I \times \Omega_{\eta(t)} \times B}\left(M\left(\nabla_{\mathbf{x}} \mathbf{u}\right) \mathbf{q}_{i} \widehat{\psi}-\sum_{j=1}^{K} \frac{A_{i j}}{4 \lambda} M \nabla_{\mathbf{q}_{j}} \widehat{\psi}\right) \cdot \nabla_{\mathbf{q}_{i}} \varphi \mathrm{d} \mathbf{q} \mathrm{d} \mathbf{x} \mathrm{d} t
\end{aligned}
$$

(c) for all $t \in I$, we have

$$
\begin{aligned}
\mathcal{E}(t) & +\mu \int_{0}^{t} \int_{\Omega_{\eta(\sigma)}}\left|\nabla_{\mathbf{x}} \mathbf{u}\right|^{2} \mathrm{~d} \mathbf{x} \mathrm{d} \sigma+\varepsilon \int_{0}^{t} \int_{\Omega_{\eta(\sigma)}}\left|\nabla_{\mathbf{x}} \Xi\right|^{2} \mathrm{~d} \mathbf{x} \mathrm{d} \sigma \\
& +4 k \varepsilon \int_{0}^{t} \int_{\Omega_{\eta(\sigma)} \times B} M\left|\nabla_{\mathbf{x}} \sqrt{\widehat{\psi}}\right|^{2} \mathrm{~d} \mathbf{q} \mathrm{d} \mathbf{x} \mathrm{d} \sigma+\frac{k A_{0}}{\lambda} \int_{0}^{t} \int_{\Omega_{\eta(\sigma)} \times B} M\left|\nabla_{\mathbf{q}} \sqrt{\widehat{\psi}}\right|^{2} \mathrm{~d} \mathbf{q} \mathrm{d} \mathbf{x} \mathrm{d} \sigma \\
& \lesssim \mathcal{E}(0)+\frac{1}{2} \int_{0}^{t}\|\mathbf{f}\|_{L^{2}\left(\Omega_{\eta(\sigma)}\right)}^{2} \mathrm{~d} \sigma+\frac{1}{2} \int_{0}^{t}\|g\|_{L^{2}(\omega)}^{2} \mathrm{~d} \sigma
\end{aligned}
$$

Remark 2.5 In the sequel, for $(t, \mathbf{x}, \mathbf{q}) \in I \times \Omega_{\eta(t)} \times B$, we shall refer to the following summand

$$
\begin{aligned}
& 4 k \varepsilon \int_{0}^{t} \int_{\Omega_{\eta(\sigma)} \times B} M\left|\nabla_{\mathbf{x}} \sqrt{\widehat{\psi}}\right|^{2} \mathrm{~d} \mathbf{q} \mathrm{d} \mathbf{x} \mathrm{d} \sigma \\
& +\frac{k A_{0}}{\lambda} \int_{0}^{t} \int_{\Omega_{\eta(\sigma)} \times B} M\left|\nabla_{\mathbf{q}} \sqrt{\widehat{\psi}}\right|^{2} \mathrm{~d} \mathbf{q} \mathrm{d} \mathbf{x} \mathrm{d} \sigma
\end{aligned}
$$


as the Fisher information. Any estimate for the energy functional (2.2) will be referred to as an energy estimate. This will include the case where formally speaking, $\psi \equiv 0$. On the other hand, an estimate involving the relative entropy (2.4) and any other function of $\psi$ such as the associated Fisher information (2.9) and the polymer number density (1.17) without any contribution from the solution $\mathbf{u}$ of the fluid equation will be referred to as an entropy estimate.

\subsection{Main Result}

The main result of this paper is the following:

Theorem 2.6 Let $\left(\mathbf{f}, g, \mathbf{u}_{0}, \eta_{0}, \eta_{1}, \widehat{\psi}_{0}\right)$ be a dataset satisfying (2.6). Then there exists a finite energy weak solution (u, $\widehat{\psi}, \eta)$ of (1.20)-(1.21), (1.30)-(1.34) and (1.7)-(1.9) on the interval $I=(0, T)$ in the sense of Definition 2.4. The number $T$ is restricted only if $\lim _{t \rightarrow T}\|\eta(t)\|_{L^{\infty}(\omega)}=L$.

Remark 2.7 We recall from the definition of $L$ in (1.1) and (1.6) that the Koiter energy does not degenerate and a self-intersection of the moving domain is excluded as long as $\|\eta(t)\|_{L^{\infty}(\omega)}$ stays strictly below $L$. If, however, we have $\lim _{t \rightarrow T}\|\eta(t)\|_{L^{\infty}(\omega)}=L$ it may happen that the Koiter energy does degenerate or that we do have a selfintersection of the shell at time $T$. In this case our existence scheme breaks.

\section{Formal Estimates for the Energy and Relative Entropy}

In this section, we formally derive energy and entropy estimates assuming that we have a sufficiently regular solution $(\mathbf{u}, \widehat{\psi}, \eta)$ of (1.20)-(1.26) and (1.7)-(1.9). Before we begin, we recall the Reynolds transport theorem which states that any vector (or scalar) $\mathbf{v}:=\mathbf{v}(t, \mathbf{x})$ of class $C_{t, \mathbf{x}}^{1}$ satisfies

$$
\begin{aligned}
\frac{\mathrm{d}}{\mathrm{d} t} \int_{\Omega_{\eta(t)}} \mathbf{v} \mathrm{d} \mathbf{x}= & \int_{\Omega_{\eta(t)}} \partial_{t} \mathbf{v} \mathrm{d} \mathbf{x}+\int_{\Omega_{\eta(t)}} \operatorname{div}_{\mathbf{x}}(\mathbf{v} \otimes \mathbf{u}) \mathrm{d} \mathbf{x}=\int_{\Omega_{\eta(t)}} \partial_{t} \mathbf{v} \mathrm{d} \mathbf{x} \\
& +\int_{\partial \Omega_{\eta(t)}}\left(\mathbf{u} \cdot \mathbf{v}_{\eta(t)}\right) \mathbf{v} \mathrm{d} \mathbf{y}
\end{aligned}
$$

in $\Omega_{\eta(t)}$ where $\mathbf{u}:=\mathbf{u}(t, \mathbf{x})$ is the velocity of the fluid (If $\mathbf{v}$ is a scalar $v$, we replace $\mathbf{v} \otimes \mathbf{u}$ with $v \mathbf{u}$ ). For a solenoidal field $\mathbf{u}$, the first equality explains the fact that the boundary of $\Omega_{\eta(t)}$, denoted by $\partial \Omega_{\eta(t)}$, moves with $\mathbf{u}$. Note that such a movement is described by the material derivative $\partial_{t}+\left(\mathbf{u} \cdot \nabla_{\mathbf{x}}\right)$. The second equation is just Gauss' theorem.

Following the arguments in Barrett and Süli (Barrett and Süli 2012c, Section 2) (for the simpler case of $K=1$ ), let us recall $\mathcal{F}(s):=s \ln s+\mathrm{e}^{-1}$ for $s>0$ so that $\mathcal{F}^{\prime}(s)=1+\ln s$ and $\mathcal{F}^{\prime \prime}(s)=\frac{1}{s}$ are well-defined. We further recall from Sect. 1.3 that

$$
M \nabla_{\mathbf{q}_{i}} \frac{1}{M}=-\frac{1}{M} \nabla_{\mathbf{q}_{i}} M=\nabla_{\mathbf{q}_{i}} U\left(\frac{1}{2}\left|\mathbf{q}_{i}\right|^{2}\right)=U^{\prime}\left(\frac{1}{2}\left|\mathbf{q}_{i}\right|^{2}\right) \mathbf{q}_{i}
$$


Now, since our flow is incompressible, by using Reynolds transport theorem (3.1),

$$
\begin{aligned}
\int_{\Omega_{\eta(t)} \times B}\left(\partial_{t} \psi+\left(\mathbf{u} \cdot \nabla_{\mathbf{x}}\right) \psi\right) \mathcal{F}^{\prime}\left(\frac{\psi}{M}\right) \mathrm{d} \mathbf{q} \mathrm{d} \mathbf{x} & =\int_{\Omega_{\eta(t)} \times B}\left(\partial_{t}+\mathbf{u} \cdot \nabla_{\mathbf{x}}\right)\left[M \mathcal{F}\left(\frac{\psi}{M}\right)\right] \mathrm{d} \mathbf{q} \mathrm{d} \mathbf{x} \\
& =\frac{\mathrm{d}}{\mathrm{d} t} \int_{\Omega_{\eta(t)} \times B} M \mathcal{F}\left(\frac{\psi}{M}\right) \mathrm{d} \mathbf{q} \mathrm{d} \mathbf{x} .
\end{aligned}
$$

Next, since the identities

$$
\begin{aligned}
\varepsilon \int_{\Omega_{\eta(t)}} \nabla_{\mathbf{x}} \psi \cdot \nabla_{\mathbf{x}} \mathcal{F}^{\prime}\left(\frac{\psi}{M}\right) \mathrm{d} \mathbf{x} & =\varepsilon \int_{\Omega_{\eta(t)}} \nabla_{\mathbf{x}} \psi \cdot \frac{M}{\psi} \nabla_{\mathbf{x}} \frac{\psi}{M} \mathrm{~d} \mathbf{x} \\
& =\varepsilon \int_{\Omega_{\eta(t)}} M \sqrt{\frac{M}{\psi}} \nabla_{\mathbf{x}} \frac{\psi}{M} \cdot \sqrt{\frac{M}{\psi}} \nabla_{\mathbf{x}} \frac{\psi}{M} \mathrm{~d} \mathbf{x} \\
& =4 \varepsilon \int_{\Omega_{\eta(t)}} M\left|\nabla_{\mathbf{x}} \sqrt{\frac{\psi}{M}}\right|^{2} \mathrm{~d} \mathbf{x}
\end{aligned}
$$

and

$$
\varepsilon \int_{\Omega_{\eta(t)}} \operatorname{div}_{\mathbf{x}}\left[\nabla_{\mathbf{x}} \psi \mathcal{F}^{\prime}\left(\frac{\psi}{M}\right)\right] \mathrm{d} \mathbf{x}=\int_{\partial \Omega_{\eta(t)}} \mathcal{F}^{\prime}\left(\frac{\psi}{M}\right) \varepsilon \nabla_{\mathbf{x}} \psi \cdot \boldsymbol{v}_{\eta(t)} \mathrm{d} \mathbf{y}_{\eta(t)}=0
$$

hold by virtue of the divergence theorem and (1.24), for the center-of-mass diffusion term, we have

$$
\varepsilon \int_{\Omega_{\eta(t)} \times B} \Delta_{\mathbf{x}} \psi \mathcal{F}^{\prime}\left(\frac{\psi}{M}\right) \mathrm{d} \mathbf{q} \mathrm{d} \mathbf{x}=-4 \varepsilon \int_{\Omega_{\eta(t)} \times B} M\left|\nabla_{\mathbf{x}} \sqrt{\frac{\psi}{M}}\right|^{2} \mathrm{~d} \mathbf{q} \mathrm{d} \mathbf{x} .
$$

Now, for each $i=1, \ldots, K$, we can use the fact that $M=0$ on $\partial B$ (see (1.16)) and the relation $s \nabla_{\mathbf{q}} \mathcal{F}^{\prime}(s)=\nabla_{\mathbf{q}} s$ to obtain

$$
\begin{aligned}
& \int_{\Omega_{\eta(t)} \times B} \operatorname{div}_{\mathbf{q}_{i}}\left(\left(\nabla_{\mathbf{x}} \mathbf{u}\right) \mathbf{q}_{i} \psi\right) \mathcal{F}^{\prime}\left(\frac{\psi}{M}\right) \mathrm{d} \mathbf{q} \mathrm{d} \mathbf{x}=-\int_{\Omega_{\eta(t)} \times B}\left(\nabla_{\mathbf{x}} \mathbf{u}\right) \mathbf{q}_{i} M \frac{\psi}{M} \cdot \nabla_{\mathbf{q}_{i}} \mathcal{F}^{\prime}\left(\frac{\psi}{M}\right) \mathrm{d} \mathbf{q} \mathrm{d} \mathbf{x} \\
& =-\int_{\Omega_{\eta(t)} \times B}\left(\nabla_{\mathbf{x}} \mathbf{u}\right) \mathbf{q}_{i} \psi \cdot\left[\frac{M \nabla_{\mathbf{q}_{i}} \psi-\psi \nabla_{\mathbf{q}_{i}} M}{M^{2}}\right] \frac{M}{\psi} \mathrm{d} \mathbf{q} \mathrm{d} \mathbf{x} \\
& =\int_{\Omega_{\eta(t)} \times B} \operatorname{div}_{\mathbf{q}_{i}}\left[\left(\nabla_{\mathbf{x}} \mathbf{u}\right) \mathbf{q}_{i}\right] \psi \mathrm{d} \mathbf{q} \mathrm{d} \mathbf{x}+\int_{\Omega_{\eta(t)} \times B}\left(\nabla_{\mathbf{x}} \mathbf{u}\right) \mathbf{q}_{i} \psi \cdot \frac{1}{M} \nabla_{\mathbf{q}_{i}} M \mathrm{~d} \mathbf{q} \mathrm{d} \mathbf{x} .
\end{aligned}
$$

However, one can check that the identity $\operatorname{div}_{\mathbf{q}_{i}}\left[\left(\nabla_{\mathbf{x}} \mathbf{u}\right) \mathbf{q}_{i}\right]=\operatorname{div}_{\mathbf{x}} \mathbf{u}=0$ holds so that in combination with (3.2), we can conclude that 


$$
\begin{aligned}
& \sum_{i=1}^{K} \int_{\Omega_{\eta(t)} \times B} \operatorname{div}_{\mathbf{q}_{i}}\left(\left(\nabla_{\mathbf{x}} \mathbf{u}\right) \mathbf{q}_{i} \psi\right) \mathcal{F}^{\prime}\left(\frac{\psi}{M}\right) \mathrm{d} \mathbf{q} \mathrm{d} \mathbf{x} \\
& \quad=-\sum_{i=1}^{K} \int_{\Omega_{\eta(t)} \times B} \psi U^{\prime}\left(\frac{1}{2}\left|\mathbf{q}_{i}\right|^{2}\right) \mathbf{q}_{i} \otimes \mathbf{q}_{i}: \nabla_{\mathbf{x}} \mathbf{u} \mathrm{d} \mathbf{q} \mathrm{d} \mathbf{x} \\
& \quad=-\sum_{i=1}^{K} \int_{\Omega_{\eta(t)}} \mathbb{T}_{i}(\psi): \nabla_{\mathbf{x}} \mathbf{u} \mathrm{d} \mathbf{x}=-\frac{1}{k} \int_{\Omega_{\eta(t)}} \mathbb{T}(\psi): \nabla_{\mathbf{x}} \mathbf{u} \mathrm{d} \mathbf{x}
\end{aligned}
$$

holds by the use of (1.18) and the observation that $\Xi^{p} \mathbb{I}: \nabla_{\mathbf{x}} \mathbf{u}=\Xi^{p} \operatorname{div}_{\mathbf{x}} \mathbf{u}=0$ for $p=1,2$. Finally, we can use that $M=0$ on $\partial B$ to obtain

$$
\begin{aligned}
& \sum_{i=1}^{K} \sum_{j=1}^{K} \frac{A_{i j}}{4 \lambda} \int_{\Omega_{\eta(t)} \times B} \operatorname{div}_{\mathbf{q}_{i}}\left(M \nabla_{\mathbf{q}_{j}} \frac{\psi}{M}\right) \mathcal{F}^{\prime}\left(\frac{\psi}{M}\right) \mathrm{d} \mathbf{q} \mathrm{d} \mathbf{x} \\
& =-\sum_{i=1}^{K} \sum_{j=1}^{K} \frac{A_{i j}}{4 \lambda} \int_{\Omega_{\eta(t)} \times B} M \nabla_{\mathbf{q}_{j}} \frac{\psi}{M} \cdot \frac{M}{\psi} \nabla_{\mathbf{q}_{i}} \frac{\psi}{M} \mathrm{~d} \mathbf{q} \mathrm{d} \mathbf{x} \\
& =-\sum_{i=1}^{K} \sum_{j=1}^{K} \frac{A_{i j}}{\lambda} \int_{\Omega_{\eta(t)} \times B} M \nabla_{\mathbf{q}_{j}} \sqrt{\frac{\psi}{M}} \cdot \nabla_{\mathbf{q}_{i}} \sqrt{\frac{\psi}{M}} \mathrm{~d} \mathbf{q} \mathrm{d} \mathbf{x}
\end{aligned}
$$

If we now collect (3.3)-(3.7) and consider the smallest eigenvalue $A_{0}>0$ of $\left(A_{i j}\right)_{i, j=1}^{K}$, then by integrating over the time interval $[0, t]$, we obtain from $(1.22)$ that

$$
\begin{aligned}
& \int_{\Omega_{\eta(t)} \times B} k M \mathcal{F}\left(\frac{\psi(t, \cdot)}{M}\right) \mathrm{d} \mathbf{q} \mathrm{d} \mathbf{x}+4 k \varepsilon \int_{0}^{t} \int_{\Omega_{\eta(\sigma)} \times B} M\left|\nabla_{\mathbf{x}} \sqrt{\frac{\psi}{M}}\right|^{2} \mathrm{~d} \mathbf{q} \mathrm{d} \mathbf{x} \mathrm{d} \sigma \\
& \quad+\frac{k A_{0}}{\lambda} \int_{0}^{t} \int_{\Omega_{\eta(\sigma) \times B}} M\left|\nabla_{\mathbf{q}} \sqrt{\frac{\psi}{M}}\right|^{2} \mathrm{~d} \mathbf{q} \mathrm{d} \mathbf{x} \mathrm{d} \sigma \\
& \leq \int_{\Omega_{\eta_{0}} \times B} k M \mathcal{F}\left(\frac{\psi_{0}}{M}\right) \mathrm{d} \mathbf{q} \mathrm{d} \mathbf{x}+\int_{0}^{t} \int_{\Omega_{\eta(\sigma)}} \mathbb{T}(\psi): \nabla_{\mathbf{x}} \mathbf{u} \mathrm{d} \mathbf{x} \mathrm{d} \sigma
\end{aligned}
$$

holds for all $t \in I$.

If we also test (1.27) with $\Xi$ and use Reynolds' transport theorem (3.1) and the boundary condition (1.28), we obtain

$$
\int_{\Omega_{\eta(t)}}|\Xi(t, \cdot)|^{2} \mathrm{~d} \mathbf{x}+\varepsilon \int_{0}^{t} \int_{\Omega_{\eta(\sigma)}}\left|\nabla_{\mathbf{x}} \Xi\right|^{2} \mathrm{~d} \mathbf{x} \mathrm{d} \sigma=\int_{\Omega_{\eta_{0}}}\left|\Xi_{0}\right|^{2} \mathrm{~d} \mathbf{x} .
$$


On the other hand, if we test (1.21) with the velocity $\mathbf{u}$, then we obtain

$$
\begin{aligned}
& \int_{\Omega_{\eta(t)}} \frac{1}{2}|\mathbf{u}(t, \cdot)|^{2} \mathrm{~d} \mathbf{x}+\mu \int_{0}^{t} \int_{\Omega_{\eta(\sigma)}}\left|\nabla_{\mathbf{x}} \mathbf{u}\right|^{2} \mathrm{~d} \mathbf{x} \mathrm{d} \sigma+\int_{0}^{t} \int_{\Omega_{\eta(\sigma)}} \mathbb{T}(\psi): \nabla_{\mathbf{x}} \mathbf{u} \mathrm{d} \mathbf{x} \mathrm{d} \sigma \\
& =\int_{\Omega_{\eta_{0}}} \frac{1}{2}\left|\mathbf{u}_{0}\right|^{2} \mathrm{~d} \mathbf{x}+\int_{0}^{t} \int_{\Omega_{\eta(\sigma)}} \mathbf{f} \cdot \mathbf{u} \mathrm{d} \mathbf{x} \mathrm{d} \sigma \\
& \quad+\int_{0}^{t} \int_{\partial \Omega_{\eta(\sigma)}}\left(2 \mu \mathbb{D}_{\mathbf{y}} \mathbf{u}+\mathbb{T}(\psi)-p \mathbb{I}\right) \mathbf{v}_{\eta(\sigma)} \cdot \mathbf{u} \mathrm{d} \mathbf{y}_{\eta(\sigma)} \mathrm{d} \sigma
\end{aligned}
$$

Finally, testing (1.7) with $\partial_{t} \eta$ yields

$$
\begin{gathered}
\int_{\omega} \frac{1}{2}\left|\partial_{t} \eta(t, \cdot)\right|^{2} \mathrm{~d} \mathbf{y}+K(\eta(t))=\int_{\omega} \frac{1}{2}\left|\eta_{1}\right|^{2} \mathrm{~d} \mathbf{y}+K\left(\eta_{0}\right) \\
+\int_{0}^{t} \int_{\omega} g \partial_{\sigma} \eta \mathrm{d} \mathbf{y} \mathrm{d} \sigma+\int_{0}^{t} \int_{\omega} \mathbf{F} \cdot \boldsymbol{v} \partial_{\sigma} \eta \mathrm{d} \mathbf{y} \mathrm{d} \sigma
\end{gathered}
$$

If we now sum up (3.8)-(3.11), then we obtain

$$
\begin{aligned}
& {\left[\int_{\Omega_{\eta(\sigma)}} \frac{1}{2}|\mathbf{u}(\sigma, \cdot)|^{2} \mathrm{~d} \mathbf{x}+\int_{\Omega_{\eta(\sigma)}}|\Xi(\sigma, \cdot)|^{2} \mathrm{~d} \mathbf{x}+\int_{\omega} \frac{1}{2}\left|\partial_{\sigma} \eta(\sigma, \cdot)\right|^{2} \mathrm{~d} \mathbf{y}+K(\eta(\sigma))\right.} \\
& \left.\quad+\int_{\Omega_{\eta(\sigma)} \times B} k M \mathcal{F}\left(\frac{\psi(\sigma, \cdot)}{M}\right) \mathrm{d} \mathbf{q} \mathrm{d} \mathbf{x}\right]_{\sigma=0}^{\sigma=t} \\
& \quad+\mu \int_{0}^{t} \int_{\Omega_{\eta(\sigma)}}\left|\nabla_{\mathbf{x}} \mathbf{u}\right|^{2} \mathrm{~d} \mathbf{x} \mathrm{d} \sigma+\varepsilon \int_{0}^{t} \int_{\Omega_{\eta(\sigma)}}\left|\nabla_{\mathbf{x}} \Xi\right|^{2} \mathrm{~d} \mathbf{x} \mathrm{d} \sigma \\
& \quad+4 k \varepsilon \int_{0}^{t} \int_{\Omega_{\eta(\sigma)} \times B} M\left|\nabla_{\mathbf{x}} \sqrt{\frac{\psi}{M}}\right|^{2} \mathrm{~d} \mathbf{q} \mathrm{d} \mathbf{x} \mathrm{d} \sigma \\
& \quad+\frac{k A_{0}}{\lambda} \int_{0}^{t} \int_{\Omega_{\eta(\sigma)} \times B} M\left|\nabla_{\mathbf{q}} \sqrt{\frac{\psi}{M}}\right|^{2} \mathrm{~d} \mathbf{q} \mathrm{d} \mathbf{x} \mathrm{d} \sigma \leq I(t)
\end{aligned}
$$

where

$$
\begin{aligned}
I(t):= & \int_{0}^{t} \int_{\Omega_{\eta(\sigma)}} \mathbf{f} \cdot \mathbf{u} \mathrm{d} \mathbf{x} \mathrm{d} \sigma+\int_{0}^{t} \int_{\omega} g \partial_{\sigma} \eta \mathrm{d} \mathbf{y} \mathrm{d} \sigma \leq \int_{0}^{t} \int_{\Omega_{\eta(\sigma)}} \frac{\vartheta}{2}|\mathbf{u}|^{2} \mathrm{~d} \mathbf{x} \mathrm{d} \sigma \\
& +\int_{0}^{t} \int_{\omega} \frac{\vartheta}{2}\left|\partial_{\sigma} \eta\right|^{2} \mathrm{~d} \mathbf{y} \mathrm{d} \sigma+\int_{0}^{t} \int_{\Omega_{\eta(\sigma)}} c_{\vartheta}|\mathbf{f}|^{2} \mathrm{~d} \mathbf{x} \mathrm{d} \sigma+\int_{0}^{t} \int_{\omega} c_{\vartheta}|g|^{2} \mathrm{~d} \mathbf{y} \mathrm{d} \sigma
\end{aligned}
$$

for any $\vartheta>0$. The energy estimate thus follow for all $t \in I$ by the use of (1.8). 


\subsection{Towards Making the Entropy Estimate Rigorous}

Since our ultimate goal is the construction of a non-negative probability function $\psi \geq 0$, even on the formal level, the derivation of the energy estimate above is still problematic. For example, $\mathcal{F}^{\prime}(s)$ and $\mathcal{F}^{\prime \prime}(s)$ are singular at $s=0$ and thus, the a priori testing of the Fokker-Planck with the first derivative of the relative entropy functional is delicate. This problem can however be fixed by using a convex regularization $\mathcal{F}_{\delta}$ to approximate $\mathcal{F}$ and then passing to the limit $\delta \rightarrow 0$. Following the arguments in Bulíček et al. (2013), for a fixed $\delta>0$, let us define $\mathcal{F}_{\delta}(s):=(s+\delta) \ln (s+\delta)+\mathrm{e}^{-1}$ for $s \geq 0$ so that $\mathcal{F}_{\delta}^{\prime}(s)=1+\ln (s+\delta)$ and $\mathcal{F}_{\delta}^{\prime \prime}(s)=\frac{1}{s+\delta}$ are well-defined even at $s=0$.

Now, because of the highly coupled nature of the macroscopic fluid system and the mesoscopic Fokker-Planck equation, we also need to introduce a preliminary smoothing in order to ultimately maintain energy balance. In this respect, we follow (Bulíček et al. 2013) and consider a smooth nonnegative function $\Gamma$ supported on the interval $(-2,2)$ such that $\Gamma(s)=1$ for all $s \in[-1,1]$. Now for $\ell \in \mathbb{N}$, we set $\Gamma_{\ell}(s):=\Gamma(s / \ell)$ and define $T_{\ell}$ and $\Lambda_{\ell}$ by

$$
T_{\ell}(s):=\int_{0}^{s} \Gamma_{\ell}(r) \mathrm{d} r, \quad \Lambda_{\ell}(s):=s \Gamma_{\ell}(s) .
$$

If we further define

$$
\begin{gathered}
T_{\delta, \ell}(s):=\int_{0}^{s} \frac{\Lambda_{\ell}(r)}{r+\delta} \mathrm{d} r=\int_{0}^{s} \frac{r \Gamma_{\ell}(r)}{r+\delta} \mathrm{d} r \\
\text { with } \quad T_{\delta, \ell} \rightarrow T_{\ell} \quad \text { in } \quad C([0, \infty)),
\end{gathered}
$$

as $\delta \rightarrow 0$, then be setting $\widehat{\psi}:=\frac{\psi}{M}$, we observe that

$$
\Lambda_{\ell}(\widehat{\psi}) \nabla_{\mathbf{q}_{i}} \mathcal{F}_{\delta}^{\prime}(\widehat{\psi})=\nabla_{\mathbf{q}_{i}} T_{\delta, \ell}(\widehat{\psi})
$$

Therefore, by using the fact that $M=0$ on $\partial B$ (see (1.16)) and the identity $\operatorname{div}_{\mathbf{q}_{i}}\left[\left(\nabla_{\mathbf{x}} \mathbf{u}\right) \mathbf{q}_{i}\right]=\operatorname{div}_{\mathbf{x}} \mathbf{u}=0$ together with (3.2), we obtain

$$
\begin{aligned}
& \int_{\Omega_{\eta(t)} \times B} \operatorname{div}_{\mathbf{q}_{i}}\left(M\left(\nabla_{\mathbf{x}} \mathbf{u}\right) \mathbf{q}_{i} \Lambda_{\ell}(\widehat{\psi})\right) \mathcal{F}_{\delta}^{\prime}(\widehat{\psi}) \mathrm{d} \mathbf{q} \mathrm{d} \mathbf{x}=-\int_{\Omega_{\eta(t)} \times B} M\left(\nabla_{\mathbf{x}} \mathbf{u}\right) \mathbf{q}_{i} \cdot \nabla_{\mathbf{q}_{i}} T_{\delta, \ell}(\widehat{\psi}) \mathrm{d} \mathbf{q} \mathrm{d} \mathbf{x} \\
& \quad=-\int_{\Omega_{\eta(t)} \times B} M T_{\delta, \ell}(\widehat{\psi}) U^{\prime}\left(\frac{1}{2}\left|\mathbf{q}_{i}\right|^{2}\right) \mathbf{q}_{i} \otimes \mathbf{q}_{i}: \nabla_{\mathbf{x}} \mathbf{u} \mathrm{d} \mathbf{q} \mathrm{d} \mathbf{x} .
\end{aligned}
$$

for the corresponding 'approximate' drag term in (1.31). By treating the rest of the terms in the modified Fokker-Planck equation (1.31) similarly to the previous subsec- 
tion, we obtain

$$
\begin{aligned}
& \frac{\mathrm{d}}{\mathrm{d} t} \int_{\Omega_{\eta(t)} \times B} k M \mathcal{F}_{\delta}(\widehat{\psi}) \mathrm{d} \mathbf{q} \mathrm{d} \mathbf{x}+k \varepsilon \int_{\Omega_{\eta(t)} \times B} M\left|\nabla_{\mathbf{x}} \widehat{\psi}\right|^{2}(\widehat{\psi}+\delta)^{-1} \mathrm{~d} \mathbf{q} \mathrm{d} \mathbf{x} \\
& \quad+\sum_{i=1}^{K} \sum_{j=1}^{K} \frac{k A_{i j}}{4 \lambda} \int_{\Omega_{\eta(t)} \times B} M \nabla_{\mathbf{q}_{j}} \widehat{\psi} \cdot \nabla_{\mathbf{q}_{i}} \widehat{\psi}(\widehat{\psi}+\delta)^{-1} \mathrm{~d} \mathbf{q} \mathrm{d} \mathbf{x} \\
& =k \sum_{i=1}^{K} \int_{\Omega_{\eta(t)} \times B} M T_{\delta, \ell}(\widehat{\psi}) U^{\prime}\left(\frac{1}{2}\left|\mathbf{q}_{i}\right|^{2}\right) \mathbf{q}_{i} \otimes \mathbf{q}_{i}: \nabla_{\mathbf{x}} \mathbf{u} \mathrm{d} \mathbf{q} \mathrm{d} \mathbf{x} .
\end{aligned}
$$

If we now consider the smallest eigenvalue $A_{0}>0$ of $\left(A_{i j}\right)_{i, j=1}^{K}$, then by integrating (3.17) over the time interval $[0, t]$, we obtain

$$
\begin{aligned}
& \int_{\Omega_{\eta(t)} \times B} k M \mathcal{F}_{\delta}(\widehat{\psi}(t, \cdot)) \mathrm{d} \mathbf{q} \mathrm{d} \mathbf{x}+k \varepsilon \int_{0}^{t} \int_{\Omega_{\eta(\sigma)} \times B} M\left|\nabla_{\mathbf{x}} \widehat{\psi}\right|^{2}(\widehat{\psi}+\delta)^{-1} \mathrm{~d} \mathbf{q} \mathrm{d} \mathbf{x} \mathrm{d} \sigma \\
& +\frac{k A_{0}}{4 \lambda} \int_{0}^{t} \int_{\Omega_{\eta(\sigma)} \times B} M\left|\nabla_{\mathbf{q}} \widehat{\psi}\right|^{2}(\widehat{\psi}+\delta)^{-1} \mathrm{~d} \mathbf{q} \mathrm{d} \mathbf{x} \mathrm{d} \sigma \leq \int_{\Omega_{\eta_{0}} \times B} k M \mathcal{F}_{\delta}\left(\widehat{\psi} \widehat{\psi}_{0}\right) \mathrm{d} \mathbf{q} \mathrm{d} \mathbf{x} \\
& +k \int_{0}^{t} \int_{\Omega_{\eta(\sigma)} \times B} \sum_{i=1}^{K} M T_{\delta, \ell}(\widehat{\psi}) U^{\prime}\left(\frac{1}{2}\left|\mathbf{q}_{i}\right|^{2}\right) \mathbf{q}_{i} \otimes \mathbf{q}_{i}: \nabla_{\mathbf{x}} \mathbf{u} \mathrm{d} \mathbf{q} \mathrm{d} \mathbf{x} \mathrm{d} \sigma .
\end{aligned}
$$

By recalling (1.18) and the observation that $\Xi^{p} \mathbb{I}: \nabla_{\mathbf{x}} \mathbf{u}=\Xi^{p} \operatorname{div}_{\mathbf{x}} \mathbf{u}=0$ for $p=1,2$, we can use (3.15) to obtain

$$
\begin{aligned}
& \underset{\delta \rightarrow 0}{\limsup _{\delta \rightarrow} k} \int_{0}^{t} \int_{\Omega_{\eta(\sigma)} \times B} \sum_{i=1}^{K} M T_{\delta, \ell}(\widehat{\psi}) U^{\prime}\left(\frac{1}{2}\left|\mathbf{q}_{i}\right|^{2}\right) \mathbf{q}_{i} \otimes \mathbf{q}_{i}: \nabla_{\mathbf{x}} \mathbf{u} \mathrm{d} \mathbf{q} \mathrm{d} \mathbf{x} \mathrm{d} \sigma \\
& \quad=k \int_{0}^{t} \int_{\Omega_{\eta(\sigma)} \times B} \sum_{i=1}^{K} M T_{\ell}(\widehat{\psi}) U^{\prime}\left(\frac{1}{2}\left|\mathbf{q}_{i}\right|^{2}\right) \mathbf{q}_{i} \otimes \mathbf{q}_{i}: \nabla_{\mathbf{x}} \mathbf{u} \mathrm{d} \mathbf{q} \mathrm{d} \mathbf{x} \mathrm{d} \sigma \\
& =\int_{0}^{t} \int_{\Omega_{\eta(\sigma)}} \mathbb{T}_{\ell}(M \widehat{\psi}): \nabla_{\mathbf{x}} \mathbf{u} \mathrm{d} \mathbf{x} \mathrm{d} \sigma
\end{aligned}
$$

where for $\widehat{\psi}=\psi / M$,

$$
\mathbb{T}_{\ell}(M \widehat{\psi})=k \sum_{i=1}^{K} \int_{B} M \nabla_{\mathbf{q}_{i}} T_{\ell}(\widehat{\psi}) \otimes \mathbf{q}_{i} \mathrm{~d} \mathbf{q}-k \Xi \mathbb{I}-\partial \Xi^{2} \mathbb{I}
$$

is a truncated extra stress tensor in the fluid system. The convergence in (3.19) together with the monotone convergence theorem and the identities

$$
\frac{M}{\widehat{\psi}}\left|\nabla_{\mathbf{x}} \widehat{\psi}\right|^{2}=4 M\left|\nabla_{\mathbf{x}} \sqrt{\widehat{\psi}}\right|^{2}, \quad \frac{M}{\widehat{\psi}}\left|\nabla_{\mathbf{q}} \widehat{\psi}\right|^{2}=4 M\left|\nabla_{\mathbf{q}} \sqrt{\widehat{\psi}}\right|^{2}
$$


allows us to finally obtain in the inequality

$$
\begin{aligned}
& \int_{\Omega_{\eta(t)} \times B} k M \mathcal{F}(\widehat{\psi}(t, \cdot)) \mathrm{d} \mathbf{q} \mathrm{d} \mathbf{x}+4 k \varepsilon \int_{0}^{t} \int_{\Omega_{\eta(\sigma)} \times B} M\left|\nabla_{\mathbf{x}} \sqrt{\widehat{\psi}}\right|^{2} \mathrm{~d} \mathbf{q} \mathrm{d} \mathbf{x} \mathrm{d} \sigma \\
& \quad+\frac{k A_{0}}{\lambda} \int_{0}^{t} \int_{\Omega_{\eta(\sigma)} \times B} M\left|\nabla_{\mathbf{q}} \sqrt{\widehat{\psi}}\right|^{2} \mathrm{~d} \mathbf{q} \mathrm{d} \mathbf{x} \mathrm{d} \sigma \leq \int_{\Omega_{\eta_{0}} \times B} k M \mathcal{F}\left(\widehat{\psi}_{0}\right) \mathrm{d} \mathbf{q} \mathrm{d} \mathbf{x} \\
& \quad+\int_{0}^{t} \int_{\Omega_{\eta(\sigma)}} \mathbb{T}_{\ell}(M \widehat{\psi}): \nabla_{\mathbf{x}} \mathbf{u} \mathrm{d} \mathbf{x} \mathrm{d} \sigma
\end{aligned}
$$

by passing to limit $\delta \rightarrow 0$ in (3.18). The last term in (3.22) above then balances with the corresponding term in the macroscopic system for the solvent.

\section{Solving the Fokker-Planck Equation}

In this section we are going to to solve to Fokker-Planck equation for a given function $\xi: I \times \omega \rightarrow(-L, L)$ and a given vector field $\mathbf{v}: I \times \Omega_{\xi} \rightarrow \mathbb{R}^{3}$ such that

$$
\xi \in C^{3}(\bar{I} \times \omega), \quad \mathbf{v} \in L^{\infty}\left(I ; W_{\operatorname{div}_{\mathbf{x}}}^{1, \infty}\left(\Omega_{\xi} ; \mathbb{R}^{3}\right)\right)
$$

Now subject to the following initial and boundary conditions for $i=1, \ldots, K$,

$$
\begin{aligned}
& {\left[\frac{1}{4 \lambda} \sum_{j=1}^{K} A_{i j} M \nabla_{\mathbf{q}_{j}} \widehat{\psi}-M\left(\nabla_{\mathbf{x}} \mathbf{v}\right) \mathbf{q}_{i} \widehat{\psi}\right] \cdot \mathbf{n}_{i}=0 \text { on } I \times \Omega_{\xi} \times \partial B_{i},} \\
& \varepsilon \nabla_{\mathbf{y}} \widehat{\psi} \cdot \boldsymbol{v}_{\xi}=\widehat{\psi}\left(\mathbf{v}-\left(\partial_{t} \xi \boldsymbol{v}\right) \circ \boldsymbol{\varphi}_{\xi}^{-1} \cdot \boldsymbol{v}_{\xi} \quad \text { on } I \times \partial \Omega_{\xi(t)} \times B\right. \text {, } \\
& \widehat{\psi}(0, \cdot, \cdot)=\widehat{\psi}_{0} \geq 0 \quad \text { in } \Omega_{\xi(0)} \times B,
\end{aligned}
$$

we aim to construct a weak solution to the Fokker-Planck equation

$$
\begin{aligned}
& \partial_{t}(M \widehat{\psi})+\left(\mathbf{v} \cdot \nabla_{\mathbf{x}}\right) M \widehat{\psi}=\varepsilon \Delta_{\mathbf{x}}(M \widehat{\psi})-\sum_{i=1}^{K} \operatorname{div}_{\mathbf{q}_{i}}\left(M\left(\nabla_{\mathbf{x}} \mathbf{v}\right) \mathbf{q}_{i} \widehat{\psi}\right) \\
& +\frac{1}{4 \lambda} \sum_{i=1}^{K} \sum_{j=1}^{K} A_{i j} \operatorname{div}_{\mathbf{q}_{i}}\left(M \nabla_{\mathbf{q}_{j}} \widehat{\psi}\right)
\end{aligned}
$$

in $I \times \Omega_{\xi(t)} \times B$. Here $\varepsilon>0$ is the center-of-mass diffusion coefficient, $\lambda>0$ is the Deborah number De and the $A_{i j}$ 's are the components of the symmetric positive definite Rouse matrix $\left(A_{i j}\right)_{i, j=1}^{K}$ whose smallest eigenvalue is $A_{0}>0$. The Maxwellian $M$ satifies (1.13)-(1.16) and $\widehat{\psi}=\widehat{\psi}(t, \mathbf{x}, \mathbf{q})$ is the unknown.

We now make precise, what we mean by $\widehat{\psi}$ being a solution of (4.2)-(4.5). 
Definition 4.1 Take $\left(\mathbf{v}, \xi, \widehat{\psi}_{0}\right)$ as a dataset such that $(\mathbf{v}, \xi)$ satisfies (4.1) and

$$
\widehat{\psi}_{0} \in L^{2}\left(\Omega_{\xi(0)} ; L_{M}^{2}(B)\right), \quad \widehat{\psi}_{0} \geq 0
$$

In addition, we assume

$$
\Xi_{0} \in L^{\infty}\left(\Omega_{\xi(0)}\right) \text { where } \Xi_{0}=\int_{B} M(\mathbf{q}) \widehat{\psi}_{0}(\cdot, \mathbf{q}) \mathrm{d} \mathbf{q} \text { in } \Omega_{\xi(0)}
$$

We call $\widehat{\psi}$ a finite energy weak solution of (4.2)-(4.5) with data (v, $\left.\xi, \widehat{\psi}_{0}\right)$ provided that:

(a) the probability density function $\widehat{\psi}$ satisfies

$$
\begin{aligned}
& \widehat{\psi} \geq 0 \text { a.e. in } I \times \Omega_{\xi(t)} \times B, \\
& \widehat{\psi} \in L^{\infty}\left(I \times \Omega_{\xi(t)} ; L_{M}^{1}(B)\right), \\
& \mathcal{F}(\widehat{\psi}) \in L^{\infty}\left(I ; L^{1}\left(\Omega_{\xi(t)} ; L_{M}^{1}(B)\right)\right), \\
& \sqrt{\widehat{\psi}} \in L^{2}\left(I ; L^{2}\left(\Omega_{\xi(t)} ; W_{M}^{1,2}(B)\right)\right) \cap L^{2}\left(I ; D^{1,2}\left(\Omega_{\xi(t)} ; L_{M}^{2}(B)\right)\right), \\
& \Xi(t, \mathbf{x})=\int_{B} M \widehat{\psi}(t, \mathbf{x}, \mathbf{q}) \mathrm{d} \mathbf{q} \in L^{\infty}\left(I \times \Omega_{\xi(t)}\right) \cap L^{2}\left(I ; W^{1,2}\left(\Omega_{\xi(t)}\right)\right) ;
\end{aligned}
$$

(b) for all $\varphi \in C^{\infty}\left(\bar{I} \times \mathbb{R}^{3} \times \bar{B}\right)$, we have

$$
\begin{aligned}
& \int_{I} \frac{\mathrm{d}}{\mathrm{d} t} \int_{\Omega_{\xi(t)} \times B} M \widehat{\psi} \varphi \mathrm{d} \mathbf{q} \mathrm{d} \mathbf{x} \mathrm{d} t \\
& \quad=\int_{I \times \Omega_{\xi(t)} \times B}\left(M \widehat{\psi} \partial_{t} \varphi+M \mathbf{v} \widehat{\psi} \cdot \nabla_{\mathbf{x}} \varphi-\varepsilon M \nabla_{\mathbf{x}} \widehat{\psi} \cdot \nabla_{\mathbf{x}} \varphi\right) \mathrm{d} \mathbf{q} \mathrm{d} \mathbf{x} \mathrm{d} t \\
& \quad+\sum_{i=1}^{K} \int_{I \times \Omega_{\xi(t)} \times B}\left(M\left(\nabla_{\mathbf{x}} \mathbf{v}\right) \mathbf{q}_{i} \widehat{\psi}-\sum_{j=1}^{K} \frac{A_{i j}}{4 \lambda} M \nabla_{\mathbf{q}_{j}} \widehat{\psi}\right) \\
& \quad \cdot \nabla_{\mathbf{q}_{i}} \varphi \mathrm{d} \mathbf{q} \mathrm{d} \mathbf{x} \mathrm{d} t
\end{aligned}
$$

(c) we have the estimate

$$
\begin{aligned}
& \|\Xi(t, \cdot)\|_{L^{\infty}\left(\Omega_{\xi(t)}\right)}^{2}+k \int_{\Omega_{\xi(t)} \times B} M \mathcal{F}(\widehat{\psi}(t, \cdot, \cdot)) \mathrm{d} \mathbf{q} \mathrm{d} \mathbf{x} \\
& \quad+\varepsilon \int_{0}^{t} \int_{\Omega_{\xi(\sigma)}}\left|\nabla_{\mathbf{x}} \Xi\right|^{2} \mathrm{~d} \mathbf{x} \mathrm{d} \sigma \\
& \quad+4 k \varepsilon \int_{0}^{t} \int_{\Omega_{\xi(\sigma)} \times B} M\left|\nabla_{\mathbf{x}} \sqrt{\widehat{\psi}}\right|^{2} \mathrm{~d} \mathbf{q} \mathrm{d} \mathbf{x} \mathrm{d} \sigma
\end{aligned}
$$




$$
\begin{aligned}
& +\frac{k A_{0}}{\lambda} \int_{0}^{t} \int_{\Omega_{\xi(\sigma)} \times B} M\left|\nabla_{\mathbf{q}} \sqrt{\widehat{\psi}}\right|^{2} \mathrm{~d} \mathbf{q} \mathrm{d} \mathbf{x} \mathrm{d} \sigma \\
\lesssim & \left\|\Xi_{0}\right\|_{L^{\infty}\left(\Omega_{\xi(0)}\right)}^{2}+k \int_{\Omega_{\xi(0)} \times B} M \mathcal{F}\left(\widehat{\psi}_{0}\right) \mathrm{d} \mathbf{q} \mathrm{d} \mathbf{x} \\
& +\int_{0}^{t} \int_{\Omega_{\xi(\sigma)}} \mathbb{T}(M \widehat{\psi}): \nabla_{\mathbf{x}} \mathbf{v} \mathrm{d} \mathbf{x} \mathrm{d} \sigma
\end{aligned}
$$

for all $t \in I$.

The main result of this section is the following:

Theorem 4.2 Assume that (v, $\left.\xi, \widehat{\psi}_{0}\right)$ satisfies (4.1) and (4.6)-(4.7). Then there exists a finite energy weak solution of (4.2)-(4.5) in the sense of Definition 4.1.

We now devote the rest of this section to the proof of Theorem 4.2. As is usually the case, we begin by establishing an approximate solution of the Fokker-Planck equation (4.5).

\subsection{A Galerkin Scheme}

We let $\left(\mathbf{v}, \xi, \widehat{\psi}_{0}\right)$ be a given dataset satisfying (4.1) and (4.6)-(4.7). Our solution $\widehat{\psi}$ to (4.5) will be derived as a limit $n \rightarrow \infty, m \rightarrow \infty, \ell \rightarrow \infty$ (in that order) of a regular solution $\widehat{\psi}^{\ell, m, n}$ where $n \in \mathbb{N}$ is a Galerkin parameter and $m \in \mathbb{N}$ and $\ell \in \mathbb{N}$ are parameters indexing a family of approximate Maxwellians and extra stress tensors respectively.

Remark 4.3 Before we begin, we remark that henceforth, for a given pair $(\mathbf{v}, \xi)$ and fixed $\ell \in \mathbb{N}$, we simply write $\widehat{\psi}^{m, n}$ in place of $\left(\widehat{\psi}^{\ell, m, n}\right)_{\ell \in \mathbb{N}}$ until when we have to pass to the limit $\ell \rightarrow \infty$. We also recall the definition of $T_{\ell}$ and $\Lambda_{\ell}$ in (3.14).

Now to begin, we first consider the following approximate form of the Maxwellian in order to avoid potential blow-up. Following Bulíček et al. (2013, Section 2.1), we approximate $M=M(\mathbf{q})$ by $M^{m}=M^{m}(\mathbf{q})$ given by

$$
M^{m}:=\bar{M}^{m}+\frac{1}{m}, \quad m \in \mathbb{N}
$$

where $\bar{M}^{m}=\bar{M}^{m}(\mathbf{q})$ is such that

$$
\lim _{m \rightarrow \infty}\left\{\left\|\bar{M}^{m}-M\right\|_{C(\bar{B}) \cap W_{0}^{1,1}(B)}+\left\|\left(\bar{M}^{m}\right)^{-1}-M^{-1}\right\|_{C\left(\mathcal{B}_{\mathbf{q}}\right)}\right\}=0
$$

for any compact set $\mathcal{B}_{\mathbf{q}} \subset B$. Next, for each $m \in \mathbb{N}$, we consider a countable family $\left(\phi_{i}^{m}\right)_{i \in \mathbb{N}}$ of eigenfunctions in $W^{1,2}(B)$ that are orthogonal in $W_{M^{m}}^{1,2}(B)$ and orthonormal in $L_{M^{m}}^{2}(B)$. We also consider a smooth basis $\left(\varpi_{j}\right)_{j \in \mathbb{N}}$ of the space $W^{1,2}\left(\mathbb{R}^{3}\right)$ along restriction to $\Omega_{\xi}$ so that $\left(\varpi_{j}\right)_{j \in \mathbb{N}}$ is a basis of the space $W^{1,2}\left(\Omega_{\xi(t)}\right)$ for any $t \in I$ 
(this, in fact, follows form the existence of an extension operator to $\mathbb{R}^{3}$ since $\xi(t)$ is smooth). For fixed $m, n \in \mathbb{N}$, our aim now is to look for a function

$$
\widehat{\psi}^{m, n}(t, \mathbf{x}, \mathbf{q})=\sum_{r=1}^{n} \sum_{l=1}^{n} f_{r l}^{m, n}(t) \phi_{r}^{m}(\mathbf{q}) \varpi_{l}(\mathbf{x})
$$

where the $f_{r l}^{m, n}$, s are the actual unknowns, such that for all $r=1, \ldots, n$ and $l=$ $1, \ldots, n$,

$$
\begin{aligned}
\frac{\mathrm{d}}{\mathrm{d} t} & \int_{\Omega_{\xi(t)} \times B} M^{m} \widehat{\psi}^{m, n} \phi_{r}^{m} \varpi_{l} \mathrm{~d} \mathbf{q} \mathrm{d} \mathbf{x} \\
= & \int_{\Omega_{\xi(t)} \times B} \mathbf{v} M^{m} \widehat{\psi}^{m, n} \cdot \nabla_{\mathbf{x}}\left(\phi_{r}^{m} \varpi_{l}\right) \mathrm{d} \mathbf{q} \mathrm{d} \mathbf{x}-\varepsilon \int_{\Omega_{\xi(t)} \times B} M^{m} \nabla_{\mathbf{x}} \widehat{\psi}^{m, n} \cdot \nabla_{\mathbf{x}}\left(\phi_{r}^{m} \varpi_{l}\right) \mathrm{d} \mathbf{q} \mathrm{d} \mathbf{x} \\
& +\sum_{i=1}^{K} \int_{\Omega_{\xi(t)} \times B} M\left(\nabla_{\mathbf{x}} \mathbf{v}\right) \mathbf{q}_{i} \Lambda_{\ell}\left(\widehat{\psi}^{m, n}\right) \cdot \nabla_{\mathbf{q}_{j}}\left(\phi_{r}^{m} \varpi_{l}\right) \mathrm{d} \mathbf{q} \mathrm{d} \mathbf{x} \\
& -\sum_{i=1}^{K} \sum_{j=1}^{K} \frac{A_{i j}}{4 \lambda} \int_{\Omega_{\xi(t)} \times B} M^{m} \nabla_{\mathbf{q}_{i}} \widehat{\psi}^{m, n} \cdot \nabla_{\mathbf{q}_{j}}\left(\phi_{r}^{m} \varpi_{l}\right) \mathrm{d} \mathbf{q} \mathrm{d} \mathbf{x}
\end{aligned}
$$

holds subject to the initial condition

$$
\begin{aligned}
& \widehat{\psi}^{m, n}(0, \cdot, \cdot)=\widehat{\psi}_{0}^{m, n}:=\sum_{r=1}^{n} \sum_{l=1}^{n}\left(\int_{\Omega_{\xi(0)} \times B} T_{\ell}\left(\widehat{\psi}_{0}^{m}\right) \phi_{r}^{m} \varpi_{l} \mathrm{~d} \mathbf{q} \mathrm{d} \mathbf{x}\right) \phi_{r}^{m}(\mathbf{q}) \varpi_{l}(\mathbf{x}), \\
& \widehat{\psi}_{0}^{m}:=\widehat{\psi}_{0} \frac{M}{M^{m}} \text { in } \quad \Omega_{\xi(0)} \times B .
\end{aligned}
$$

Equation (4.13) is a system of ODEs with continuous coefficients which can be solved locally in time. We start by showing uniform a priori estimates for $\widehat{\psi}^{m, n}$ which will imply global solvability of (4.13). To obtain these estimates, we will take $\widehat{\psi}^{m, n}$ as test function in (4.13). Using Reynolds' transport theorem (3.1) we obtain

$$
\begin{aligned}
\frac{\mathrm{d}}{\mathrm{d} t} & \int_{\Omega_{\xi(t)} \times B} M^{m}\left|\widehat{\psi}^{m, n}\right|^{2} \mathrm{~d} \mathbf{q} \mathrm{d} \mathbf{x}-\int_{\Omega_{\xi(t)} \times B} M^{m} \widehat{\psi}^{m, n} \partial_{t} \widehat{\psi}^{m, n} \mathrm{~d} \mathbf{q} \mathrm{d} \mathbf{x} \\
= & \frac{1}{2} \frac{\mathrm{d}}{\mathrm{d} t} \int_{\Omega_{\xi(t)} \times B} M^{m}\left|\widehat{\psi}^{m, n}\right|^{2} \mathrm{~d} \mathbf{q} \mathrm{d} \mathbf{x} \\
& +\frac{1}{2} \int_{\partial \Omega_{\xi(t)} \times B} M^{m}\left|\widehat{\psi}^{m, n}\right|^{2}\left(\partial_{t} \xi \boldsymbol{v}\right) \circ \boldsymbol{\varphi}_{\xi}^{-1} \cdot \boldsymbol{v}_{\xi} \mathrm{d} \mathbf{q} \mathrm{d} \mathbf{y},
\end{aligned}
$$

whereas the divergence theorem yields

$$
\int_{\Omega_{\xi(t)} \times B} \mathbf{v} M^{m} \widehat{\psi}^{m, n} \cdot \nabla_{\mathbf{x}} \widehat{\psi}^{m, n} \mathrm{~d} \mathbf{q} \mathrm{d} \mathbf{x}=\frac{1}{2} \int_{\partial \Omega_{\xi(t)} \times B} M^{m}\left|\widehat{\psi}^{m, n}\right|^{2}\left(\mathbf{v} \cdot \boldsymbol{v}_{\xi}\right) \mathrm{d} \mathbf{q} \mathrm{d} \mathbf{y}
$$


since $\operatorname{div}_{\mathbf{x}} \mathbf{v}=0$. By treating the rest of the terms in (4.13) in a straightforward manner and integrating over the interval $(0, t)$, we obtain

$$
\begin{aligned}
& \left.\frac{1}{2} \int_{\Omega_{\xi(\sigma)} \times B} M^{m}\left|\widehat{\psi}^{m, n}(\sigma)\right|^{2} \mathrm{~d} \mathbf{q} \mathrm{d} \mathbf{x}\right]_{\sigma=0}^{\sigma=t} \\
& +\sum_{i=1}^{K} \sum_{j=1}^{K} \frac{A_{i j}}{4 \lambda} \int_{0}^{t} \int_{\Omega_{\xi(\sigma)} \times B} M^{m} \nabla_{\mathbf{q}_{i}} \widehat{\psi}^{m, n} \cdot \nabla_{\mathbf{q}_{j}} \widehat{\psi}^{m, n} \mathrm{~d} \mathbf{q} \mathrm{d} \mathbf{x} \mathrm{d} \sigma \\
& +\varepsilon \int_{0}^{t} \int_{\Omega_{\xi(\sigma) \times B}} M^{m}\left|\nabla_{\mathbf{x}} \widehat{\psi}^{m, n}\right|^{2} \mathrm{~d} \mathbf{q} \mathrm{d} \mathbf{x} \mathrm{d} \sigma \\
& =\sum_{i=1}^{K} \int_{0}^{t} \int_{\Omega_{\xi(\sigma)} \times B} M^{m}\left(\nabla_{\mathbf{x}} \mathbf{v}\right) \mathbf{q}_{i} \Lambda_{\ell}\left(\widehat{\psi}^{m, n}\right) \cdot \nabla_{\mathbf{q}_{i}} \widehat{\psi}^{m, n} \mathrm{~d} \mathbf{q} \mathrm{d} \mathbf{x} \mathrm{d} \sigma \\
& +\frac{1}{2} \int_{0}^{t} \int_{\partial \Omega_{\xi(\sigma) \times B}} M^{m}\left|\widehat{\psi}^{m, n}\right|^{2}\left(\mathbf{v} \cdot \boldsymbol{v}_{\xi}\right) \mathrm{d} \mathbf{q} \mathrm{d} \mathbf{y} \mathrm{d} \sigma \\
& -\frac{1}{2} \int_{0}^{t} \int_{\partial \Omega_{\xi(\sigma) \times B}} M^{m}\left|\widehat{\psi}^{m, n}\right|^{2}\left(\partial_{\sigma} \xi \boldsymbol{v}\right) \circ \boldsymbol{\varphi}_{\xi}^{-1} \cdot \boldsymbol{v}_{\xi} \mathrm{d} \mathbf{q} \mathrm{d} \mathbf{y} \mathrm{d} \sigma \\
& =:(I)^{m, n}+(I I)^{m, n}+(I I I)^{m, n}
\end{aligned}
$$

for all $t \in I$. As a consequence of Young's inequality, the definitions of $\Lambda_{\ell}$ and $M^{m}$, boundedness of $\nabla_{\mathbf{X}} \mathbf{v}$ and the boundedness of $\left|\mathbf{q}_{i}\right|^{2}$ which holds for each $i=1, \ldots, K$, we obtain the estimate

$$
(I)^{m, n} \leq \delta \int_{0}^{t} \int_{\Omega_{\xi(\sigma)} \times B} M^{m}\left|\nabla_{\mathbf{q}} \widehat{\psi}^{m, n}\right|^{2} \mathrm{~d} \mathbf{q} \mathrm{d} \mathbf{x} \mathrm{d} \sigma+c_{\delta} \int_{0}^{t} \int_{\Omega_{\xi(\sigma)} \times B} M^{m}\left|\widehat{\psi}^{m, n}\right|^{2} \mathrm{~d} \mathbf{q} \mathrm{d} \mathbf{x} \mathrm{d} \sigma
$$

uniformly in $m, n \in \mathbb{N}$ for any $\delta>0$ recalling the definition of $\Lambda_{\ell}$. In order to estimate $(I I)^{m, n}$, we use the trace embedding $W^{1 / 2,2}\left(\Omega_{\xi(t)}\right) \hookrightarrow L^{2}\left(\partial \Omega_{\xi(t)}\right)$. The constant in this embedding depends on the $W_{\mathbf{x}}^{1, \infty}$-norm of $\xi$. Employing also an interpolation argument and using the regularity of $\mathbf{v}$ again, we obtain

$$
\begin{aligned}
(I I)^{m, n} & \lesssim \int_{0}^{t} \int_{B} M^{m}\left\|\widehat{\psi}^{m, n}\right\|_{L^{2}\left(\partial \Omega_{\xi(\sigma)}\right)}^{2} \mathrm{~d} \mathbf{q} \mathrm{d} \sigma \lesssim \int_{0}^{t} \int_{B} M^{m}\left\|\widehat{\psi}^{m, n}\right\|_{W^{1 / 2,2}\left(\Omega_{\xi(\sigma)}\right)}^{2} \mathrm{~d} \mathbf{q} \mathrm{d} \sigma \\
& \leq \delta \int_{0}^{t} \int_{\Omega_{\xi(\sigma)} \times B} M^{m}\left|\nabla_{\mathbf{x}} \widehat{\psi}^{m, n}\right|^{2} \mathrm{~d} \mathbf{q} \mathrm{d} \mathbf{x} \mathrm{d} \sigma+c_{\delta} \int_{0}^{t} \int_{\Omega_{\xi(\sigma)} \times B} M^{m}\left|\widehat{\psi}^{m, n}\right|^{2} \mathrm{~d} \mathbf{q} \mathrm{d} \mathbf{x} \mathrm{d} \sigma
\end{aligned}
$$

uniformly in $m, n \in \mathbb{N}$ for any $\delta>0$. By employing the regularity of $\xi$ in place of $\mathbf{v}$, we can derive the same estimate for $(I I I)^{m, n}$. If we now consider the smallest eigenvalue $A_{0}>0$ of $\left(A_{i j}\right)_{i, j=1}^{K}$, absorb the small $\delta$-terms into the corresponding 
terms on the left-hand side of (4.15) and apply Gronwall's lemma, then we obtain

$$
\begin{aligned}
& \int_{\Omega_{\xi(t)} \times B} M^{m}\left|\widehat{\psi}^{m, n}\right|^{2} \mathrm{~d} \mathbf{q} \mathrm{d} \mathbf{x}+\int_{0}^{t} \int_{\Omega_{\xi(\sigma)} \times B} M^{m}\left|\nabla_{\mathbf{x}} \widehat{\psi}^{m, n}\right|^{2} \mathrm{~d} \mathbf{q} \mathrm{d} \mathbf{x} \mathrm{d} \sigma \\
& \quad+\int_{0}^{t} \int_{\Omega_{\xi(\sigma)} \times B} M^{m}\left|\nabla_{\mathbf{q}} \widehat{\psi}^{m, n}\right|^{2} \mathrm{~d} \mathbf{q} \mathrm{d} \mathbf{x} \mathrm{d} \sigma \lesssim \int_{\Omega_{\xi(0)} \times B} M^{m}\left|\widehat{\psi}_{0}^{m, n}\right|^{2} \mathrm{~d} \mathbf{q} \mathrm{d} \mathbf{x}
\end{aligned}
$$

uniformly in $m, n \in \mathbb{N}$ for all $t \in I$ with a constant that depends only on $A_{0}, \varepsilon>0$, $\mathbf{v}$ and $\xi$. Note that (4.16) implies that there is a global-in-time solution to (4.13). Furthermore, we can use the fact that $\frac{1}{m} \leq M^{m} \leq c$, where $c>0$ is a constant independent of $m$ to conclude from (4.16) that

$$
\begin{aligned}
& \sup _{t \in I} \int_{\Omega_{\xi(t)} \times B}\left|\widehat{\psi}^{m, n}\right|^{2} \mathrm{~d} \mathbf{q} \mathrm{d} \mathbf{x}+\int_{I} \int_{\Omega_{\xi(t)} \times B}\left|\nabla_{\mathbf{x}} \widehat{\psi}^{m, n}\right|^{2} \mathrm{~d} \mathbf{q} \mathrm{d} \mathbf{x} \mathrm{d} t \\
& \quad+\int_{I} \int_{\Omega_{\xi(t)} \times B}\left|\nabla_{\mathbf{q}} \widehat{\psi}^{m, n}\right|^{2} \mathrm{~d} \mathbf{q} \mathrm{d} \mathbf{x} \mathrm{d} t \lesssim m
\end{aligned}
$$

where the constant additionally depends only on $A_{0}, \varepsilon>0$, $\mathbf{v}$ and $\xi$.

\subsection{Regularity and Identification of the First Limits}

To begin this subsection, we first note that since the estimate (4.17) holds uniformly in $n \in \mathbb{N}$, it follows that there exist

$$
\begin{aligned}
\widehat{\psi}^{m} \in L^{\infty}\left(I ; L^{2}\left(\Omega_{\xi(t)} ; L^{2}(B)\right)\right) & \cap L^{2}\left(I ; D^{1,2}\left(\Omega_{\xi(t)} ; L^{2}(B)\right)\right) \\
& \cap L^{2}\left(I ; L^{2}\left(\Omega_{\xi(t)} ; W^{1,2}(B)\right)\right)
\end{aligned}
$$

such that $\widehat{\psi}^{m, n}$ converges weakly-(*) to $\widehat{\psi}^{m}$ as $n \rightarrow \infty$. The limit $\widehat{\psi}^{m}$ satisfies both (4.16) and (4.17). Furthermore, since $\frac{3}{5}=3\left(\frac{1}{2}-\frac{3}{10}\right)$, it follows from the GagliardoNirenberg interpolation inequality that for all $t \in I$, the estimate

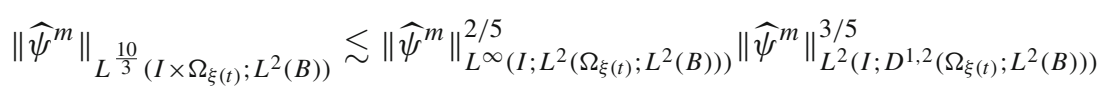

holds for a constant depending only on $\xi$. Thus, given (4.18), it follows that

$$
\widehat{\psi}^{m} \in L^{\frac{10}{3}}\left(I \times \Omega_{\xi(t)} ; L^{2}(B)\right) .
$$

Although the original equation (4.5) is linear in $\psi$, the regularised equation (which we approximate by (4.13)) is not due to the modified drag-term. Hence, we need compactness to pass to the limit. On account of the moving shell, the standard argument based on Aubin-Lions lemma does not apply. Also, we cannot localise the equation on the Galerkin level (this will be done later in Sects. 4.5 and 6.2). Hence, we modify the compactness approach from Lengeler and Růžička (2014) for our purposes and prove that 


$$
\int_{I \times \Omega_{\xi(t)} \times B} M^{m}\left|\widehat{\psi}^{m, n}\right|^{2} \mathrm{~d} \mathbf{q} \mathrm{d} \mathbf{x} \mathrm{d} t \rightarrow \int_{I \times \Omega \xi(t) \times B} M^{m}\left|\widehat{\psi}^{m}\right|^{2} \mathrm{~d} \mathbf{q} \mathrm{d} \mathbf{x} \mathrm{d} t
$$

as $n \rightarrow \infty$. In order to do so, we consider for $\varphi \in W^{1,2}\left(\mathbb{R}^{3} \times B\right)$ with $\|\varphi\|_{W_{(\mathbf{x}, \mathbf{q})}^{1,2}} \leq 1$, the functions

$$
\begin{aligned}
c_{\varphi, n}(t) & =\int_{\Omega_{\xi(t)} \times B} M^{m} \widehat{\psi}^{m, n}(t) \Pi_{n}^{\mathbf{q}} \Pi_{n}^{\mathbf{x}} \varphi \mathrm{d} \mathbf{q} \mathrm{d} \mathbf{x}, \\
c_{\varphi}(t) & =\int_{\Omega_{\xi(t)} \times B} M^{m} \widehat{\psi}^{m}(t) \varphi \mathrm{d} \mathbf{q} \mathrm{d} \mathbf{x},
\end{aligned}
$$

where $\Pi_{n}^{\mathbf{q}}$ and $\Pi_{n}^{\mathbf{x}}$ are $L^{2}$-projections onto $\left(\phi_{i}\right)_{i=1}^{n}$ and $\left(\varpi_{i}\right)_{i=1}^{n}$ respectively. We aim to show

$$
\left\|c_{\varphi, n}\right\|_{C^{0,1 / \chi^{\prime}(\bar{I})}} \lesssim 1
$$

for some $\chi>1$ uniformly in $n$ and $\varphi$. This follows by inserting $\Pi_{n}^{\mathbf{q}} \Pi_{n}^{\mathbf{x}} \varphi$ into (4.13) and integrating in time. The result is then a consequence of the a priori estimate from (4.17) and the continuity of $\Pi_{n}^{\mathbf{q}}$ and $\Pi_{n}^{\mathbf{x}}$. Taking a subsequence and applying ArceláAscoli's theorem, we can conclude that $c_{\varphi, n}$ converges uniformly in $\bar{I}$. Using (4.21) we will prove that the function

$$
t \mapsto \sup _{\|\varphi\|_{W_{(\mathbf{x}, \mathbf{q})}^{1,2}}^{1,2}}\left(c_{\varphi, n}(t)-c_{\varphi}(t)\right)
$$

converges uniformly in $\bar{I}$. First of all we extend $\widehat{\psi}^{m, n}(t, \cdot, \mathbf{q})$ and $\widehat{\psi}^{m}(t, \cdot, \mathbf{q})$ from $\Omega_{\xi(t)}$ to $S_{L}$ and use the compact embedding $L^{2}\left(S_{L} \times B\right) \hookrightarrow W^{-1,2}\left(S_{L} \times B\right)$ to conclude that (after taking a diagonal sequence)

$$
\widehat{\psi}^{m, n}(t) \rightarrow \widehat{\psi}^{m}(t) \quad \text { in } \quad W^{-1,2}\left(\Omega_{\xi(t)} \times B\right)
$$

for all $t \in I_{0}$, where $I_{0} \subset I$ is a dense subset. Consequently, we have for each such $t$

$$
\begin{aligned}
\left|c_{\varphi, n}(t)-c_{\varphi}(t)\right| \leq & \left|\int_{\Omega_{\xi(t)} \times B} M^{m}\left(\widehat{\psi}^{m, n}(t)-\widehat{\psi}^{m}(t)\right) \Pi_{n}^{\mathbf{q}} \Pi_{n}^{\mathbf{x}} \varphi \mathrm{d} \mathbf{q} \mathrm{d} \mathbf{x}\right| \\
& +\left|\int_{\Omega_{\xi(t)} \times B} M^{m} \widehat{\psi}^{m}(t)\left(\Pi_{n}^{\mathbf{q}} \Pi_{n}^{\mathbf{x}} \varphi-\varphi\right) \mathrm{d} \mathbf{q} \mathrm{d} \mathbf{x}\right| \\
& \leq c\left(\left\|\widehat{\psi}^{m, n}(t)-\widehat{\psi}^{m}(t)\right\|_{W_{(\mathbf{x}, \mathbf{q})}^{-1,2}}+\left\|\widehat{\psi}^{m}(t)\right\|_{L_{(\mathbf{x}, \mathbf{q})}^{2}}\left\|\Pi_{n}^{\mathbf{q}} \Pi_{n}^{\mathbf{x}} \varphi-\varphi\right\|_{L_{(\mathbf{x}, \mathbf{q})}^{2}}\right) \\
& \longrightarrow 0
\end{aligned}
$$


uniformly in $\varphi$. Combining this with (4.21) and boundedness of $M^{m}$ easily yields (4.22). On account of (4.17) and (4.22), we have

$$
\begin{aligned}
\int_{I}\left(c_{\widehat{\psi}^{m, n}, n}(t)-c_{\widehat{\psi}^{m, n}}(t)\right) \mathrm{d} t & =\int_{I}\left\|\widehat{\psi}^{m, n}\right\|_{W_{(\mathbf{x}, \mathbf{q})}^{1,2}} \frac{\left(c_{\widehat{\psi}^{m, n}, n}(t)-c_{\widehat{\psi}^{m, n}}(t)\right)}{\left\|\widehat{\psi}^{m, n}\right\|_{W_{(\mathbf{x}, \mathbf{q})}^{1,2}}} \mathrm{~d} t \\
& \leq\left(\int_{I}\left\|\widehat{\psi}^{m, n}\right\|_{W_{(\mathbf{x}, \mathbf{q})}^{1,2}}^{2} \mathrm{~d} t\right)^{\frac{1}{2}}\left(\int_{I\|\varphi\|_{W_{(\mathbf{x}, \mathbf{q})}^{1,2}} \leq 1} \sup \left(c_{\varphi, n}(t)-c_{\varphi}(t)\right)^{2} \mathrm{~d} t\right)^{\frac{1}{2}} \\
& \longrightarrow 0
\end{aligned}
$$

as $n \rightarrow \infty$ using also (4.17). By combining this with the weak convergence of $\widehat{\psi}^{m, n}$ from (4.18), we obtain (4.20). Since $M^{m}$ is strictly positive and bounded, one can now easily pass to the limit in (4.13) and conclude that the limit $\widehat{\psi}^{m}$ satisfies

$$
\begin{aligned}
& \int_{I} \frac{\mathrm{d}}{\mathrm{d} t} \int_{\Omega_{\xi(t)} \times B} M^{m} \widehat{\psi}^{m} \varphi \mathrm{d} \mathbf{q} \mathrm{d} \mathbf{x} \mathrm{d} t=\int_{I \times \Omega_{\xi(t)} \times B} M^{m} \widehat{\psi}^{m} \partial_{t} \varphi \mathrm{d} \mathbf{q} \mathrm{d} \mathbf{x} \mathrm{d} t \\
& \quad+\int_{I \times \Omega_{\xi(t)} \times B}\left(\mathbf{v} M^{m} \widehat{\psi}^{m}-\varepsilon M^{m} \nabla_{\mathbf{x}} \widehat{\psi}^{m}\right) \cdot \nabla_{\mathbf{x}} \varphi \mathrm{d} \mathbf{q} \mathrm{d} \mathbf{x} \mathrm{d} t \\
& \quad+\sum_{i=1}^{K} \int_{I \times \Omega_{\xi(t)} \times B}\left(M\left(\nabla_{\mathbf{x}} \mathbf{v}\right) \mathbf{q}_{i} \Lambda_{\ell}\left(\widehat{\psi}^{m}\right)-\sum_{j=1}^{K} \frac{A_{i j}}{4 \lambda} M^{m} \nabla_{\mathbf{q}_{j}} \widehat{\psi}^{m}\right) \cdot \nabla_{\mathbf{q}_{i}} \varphi \mathrm{d} \mathbf{q} \mathrm{d} \mathbf{x} \mathrm{d} t
\end{aligned}
$$

for all $\varphi \in C^{\infty}\left(\bar{I} \times \mathbb{R}^{3} \times \bar{B}\right)$. Noticing the duality

$$
\left(L^{2}\left(I ; W^{1,2}\left(\Omega_{\xi(t)} \times B\right)\right)\right)^{\prime} \cong L^{2}\left(I ; W^{-1,2}\left(\Omega_{\xi(t)} \times B\right)\right),
$$

cf. (Nägele et al. 2017, page 8), we can rewrite (4.23) as

$$
\begin{aligned}
& \left\langle\partial_{t}\left(M^{m} \widehat{\psi}^{m}\right), \varphi\right\rangle_{t}=\int_{\Omega_{\xi(t)} \times B}\left(\mathbf{v} M^{m} \widehat{\psi}^{m}-\varepsilon M^{m} \nabla_{\mathbf{x}} \widehat{\psi}^{m}\right) \cdot \nabla_{\mathbf{x}} \varphi \mathrm{d} \mathbf{q} \mathrm{d} \mathbf{x} \\
& +\sum_{i=1}^{K} \int_{\Omega_{\xi(t)} \times B}\left(M\left(\nabla_{\mathbf{x}} \mathbf{v}\right) \mathbf{q}_{i} \Lambda_{\ell}\left(\widehat{\psi}^{m}\right)-\sum_{j=1}^{K} \frac{A_{i j}}{4 \lambda} M^{m} \nabla_{\mathbf{q}_{j}} \widehat{\psi}^{m}\right) \cdot \nabla_{\mathbf{q}_{i}} \varphi \mathrm{d} \mathbf{q} \mathrm{d} \mathbf{x} \\
& \quad-\int_{\partial \Omega_{\xi(t)} \times B}\left(\partial_{t} \xi \mathbf{v}\right) \circ \boldsymbol{\varphi}_{\xi}^{-1} \cdot \boldsymbol{v}_{\xi} M^{m} \widehat{\psi}^{m} \varphi \mathrm{d} \mathbf{q} \mathrm{d} \mathbf{y}
\end{aligned}
$$

for $t \in I$ and all $\varphi \in W^{1,2}\left(\Omega_{\xi(t)} \times B\right)$. Here $\langle\cdot, \cdot\rangle_{t}$ denotes the duality pairing between $W^{-1,2}\left(\Omega_{\xi(t)} \times B\right)$ and $W^{1,2}\left(\Omega_{\xi(t)} \times B\right)$. For various estimates, we need the formula

$$
\begin{aligned}
& \int_{0}^{\tau}\left\langle\partial_{t}\left(M^{m} \widehat{\psi}^{m}\right), f^{\prime}\left(\widehat{\psi}^{m}\right)\right\rangle_{t} \mathrm{~d} t=\int_{\Omega_{\xi(\tau)} \times B} M^{m} f\left(\widehat{\psi}^{m}(\tau)\right) \mathrm{d} \mathbf{x} \mathrm{d} \mathbf{q}-\int_{\Omega_{\xi(0)} \times B} M^{m} f\left(\widehat{\psi}^{m}(0)\right) \mathrm{d} \mathbf{x} \mathrm{d} \mathbf{q} \\
& -\int_{0}^{\tau} \int_{\partial \Omega_{\xi(t)} \times B} M^{m} f\left(\widehat{\psi}^{m}\right)\left(\partial_{t} \xi \boldsymbol{v}\right) \circ \boldsymbol{\varphi}_{\xi}^{-1} \cdot \boldsymbol{v}_{\xi} \mathrm{d} \mathbf{q} \mathrm{d} \mathbf{y} \mathrm{d} t
\end{aligned}
$$


for a.e. $\tau \in I$, provided $f$ is a Lipschitz-function. In order to prove (4.26), we can define a temporal regularisation operator $\mathscr{S}_{\varrho}$ on $L^{2}\left(I ; W^{1,2}\left(\Omega_{\xi(t)} \times B\right)\right)$ by

$$
\mathscr{S}_{\varrho} \phi=\left.\left(\mathscr{E}_{\xi}(t) \phi\right)_{\varrho}\right|_{I \times \Omega_{\xi} \times B}
$$

where $\mathscr{E}_{\xi}(t): W^{1,2}\left(\Omega_{\xi(t)}\right) \rightarrow W^{1,2}\left(\mathbb{R}^{3}\right)$ is the standard extension operator (note that the boundary of $\Omega_{\xi(t)}$ is smooth) and $(\cdot)_{\varrho}$ denotes a temporal regularisation which is symmetric and commutes with derivatives. We clearly have

$$
\mathscr{S}_{\varrho} \phi \rightarrow \phi \quad \text { in } L^{2}\left(I ; W^{1,2}\left(\Omega_{\xi(t)} \times B\right)\right)
$$

for $\phi \in L^{2}\left(I ; W^{1,2}\left(\Omega_{\xi(t)} \times B\right)\right)$. Thus, for $\varphi \in C^{\infty}\left(\bar{I} \times \mathbb{R}^{3}\right)$ with $\varphi(0, \cdot)=\varphi(T, \cdot)=$ 0 , we have

$$
\begin{aligned}
\int_{I}\left\langle\partial_{t} \mathscr{S}_{\varrho}\left(M^{m} \widehat{\psi}^{m}\right), \varphi\right\rangle_{t} \mathrm{~d} t= & -\int_{I} \int_{\Omega_{\xi(t)} \times B} \mathscr{S}_{\varrho}\left(M^{m} \widehat{\psi}^{m}\right) \partial_{t} \varphi \mathrm{d} \mathbf{q} \mathrm{d} \mathbf{x} \mathrm{d} t \\
& -\int_{I} \int_{\partial \Omega_{\xi(t)} \times B}\left(\partial_{t} \xi \boldsymbol{v}\right) \circ \boldsymbol{\varphi}_{\xi}^{-1} \cdot \boldsymbol{v}_{\xi} \mathscr{S}_{\varrho}\left(M^{m} \widehat{\psi}^{m}\right) \varphi \mathrm{d} \mathbf{q} \mathrm{d} \mathbf{y} \mathrm{d} t \\
= & -\int_{I} \int_{\Omega_{\xi(t)} \times B} M^{m} \widehat{\psi}^{m} \partial_{t} \mathscr{S}_{\varrho} \varphi \mathrm{d} \mathbf{q} \mathrm{d} \mathbf{x} \mathrm{d} t \\
& -\int_{I} \int_{\partial \Omega_{\xi(t)} \times B}\left(\partial_{t} \xi \boldsymbol{v}\right) \circ \boldsymbol{\varphi}_{\xi}^{-1} \cdot \boldsymbol{v}_{\xi} \mathscr{S}_{\varrho}\left(M^{m} \widehat{\psi}^{m}\right) \varphi \mathrm{d} \mathbf{q} \mathrm{d} \mathbf{y} \mathrm{d} t
\end{aligned}
$$

Using $\mathscr{S}_{\varrho} \varphi$ as a test-function in (4.23), the latter formula also make sense for $\varphi \in$ $L^{2}\left(I ; W^{1,2}\left(\Omega_{\xi(t)} \times B\right)\right)$. Moreover, we obtain

$$
\partial_{t} \mathscr{S}_{\varrho}\left(M^{m} \widehat{\psi}^{m}\right) \rightarrow \partial_{t}\left(M^{m} \widehat{\psi}^{m}\right) \quad \text { in } \quad L^{2}\left(I ; W^{-1,2}\left(\Omega_{\xi(t)} \times B\right)\right)
$$

as a consequence of (4.27). Combining (4.27) and (4.28) finally proves (4.26).

With (4.26) at hand we can justify taking $\left(\widehat{\psi}^{m}\right)_{-}:=\min \left\{0, \widehat{\psi}^{m}\right\}$ as a test function in (4.25). Indeed, choosing $f(s)=s_{-}:=\min \{0, s\}$ we obtain in analogy with (4.17),

$$
\begin{aligned}
& \sup _{t \in I} \int_{\Omega_{\xi(t)} \times B}\left|\left(\widehat{\psi}^{m}\right)_{-}\right|^{2} \mathrm{~d} \mathbf{q} \mathrm{d} \mathbf{x}+\int_{I} \int_{\Omega_{\xi(t)} \times B}\left|\nabla_{\mathbf{x}}\left(\widehat{\psi}^{m}\right)_{-}\right|^{2} \mathrm{~d} \mathbf{q} \mathrm{d} \mathbf{x} \mathrm{d} t \\
& \quad+\int_{I} \int_{\Omega_{\xi(t)} \times B}\left|\nabla_{\mathbf{q}}\left(\widehat{\psi}^{m}\right)_{-}\right|^{2} \mathrm{~d} \mathbf{q} \mathrm{d} \mathbf{x} \mathrm{d} t \lesssim_{m} 0
\end{aligned}
$$

where the constant depends only on $A_{0}, \varepsilon>0, \mathbf{v}$ and $\xi$. As opposed to (4.17), the right hand side of (4.29) is zero because $\left.\left(\widehat{\psi}^{m}\right)_{-}\right|_{t=0}=0$ since $T_{\ell}\left(\widehat{\psi}_{0}^{m}\right) \geq 0$. We can therefore conclude that $\left(\widehat{\psi}^{m}\right)_{-} \equiv 0$ in $I \times \Omega_{\xi(t)} \times B$ and thus

$$
\widehat{\psi}^{m} \geq 0 \text { a.e. in } I \times \Omega_{\xi(t)} \times B ;
$$

the minimum principle. 


\subsection{An Auxiliary Problem: The Dumped Continuity Equation}

For the ultimate purpose of estimating the Kramer's expansion of the extra stress tensor $\mathbb{T}(\psi)$, we also need to consider the solution $\Xi^{m}$ of a dumped continuity equation for the weighted average of $\widehat{\psi}^{m}$. In particular, by setting $\Xi^{m}:=\int_{B} M^{m} \widehat{\psi}^{m} \mathrm{dq}$ where $m \in \mathbb{N}$ is fixed and choosing the test-function in (4.23) to be independent of $\mathbf{q}$, we obtain

$$
\begin{aligned}
& \int_{I} \frac{\mathrm{d}}{\mathrm{d} t} \int_{\Omega_{\xi(t)}} \Xi^{m} \varphi \mathrm{d} \mathbf{x} \mathrm{d} t \\
& \quad=\int_{I \times \Omega_{\xi(t)}}\left(\Xi^{m} \partial_{t} \varphi+\mathbf{v} \Xi^{m} \cdot \nabla_{\mathbf{x}} \varphi-\varepsilon \nabla_{\mathbf{x}} \Xi^{m} \cdot \nabla_{\mathbf{x}} \varphi\right) \mathrm{d} \mathbf{x} \mathrm{d} t
\end{aligned}
$$

for all $\varphi \in C^{\infty}\left(\bar{I} \times \mathbb{R}^{3}\right)$. We supplement (4.31) with the initial condition

$$
\Xi^{m}(0, \cdot)=\Xi_{0}^{m}:=\int_{B} M^{m} T_{\ell}\left(\widehat{\psi}_{0}^{m}\right) \mathrm{d} \mathbf{q} \leq \int_{B} M \widehat{\psi}_{0} \mathrm{~d} \mathbf{q}=\Xi_{0} .
$$

As shown in Breit and Schwarzacher (2018, Theorem 3.1), there is a unique solution to $(4.31)$ in the class

$$
L^{\infty}\left(I ; L^{2}\left(\Omega_{\xi(t)}\right)\right) \cap L^{2}\left(I ; W^{1,2}\left(\Omega_{\xi(t)}\right)\right)
$$

and it satisfies the estimate

$$
\left\|\Xi^{m}\right\|_{L^{\infty}\left(I ; L^{2}\left(\Omega_{\xi(t)}\right)\right)}^{2}+\left\|\nabla_{\mathbf{x}} \Xi^{m}\right\|_{L^{2}\left(I \times \Omega_{\xi(t)}\right)}^{2} \lesssim\left\|\Xi_{0}\right\|_{L^{2}\left(\Omega_{\xi(0)}\right)}^{2}
$$

with a constant depending only on the center-of-mass diffusion coefficient $\varepsilon>0$, $\xi(t)$ and $\mathbf{v}$. It is also shown in Breit and Schwarzacher (2018, Theorem 3.1) that the unique solution to (4.31) satisfies a renormalised formulation which reads as follows. For $\theta \in C^{2}([0, \infty) ;[0, \infty))$ with $\theta^{\prime}(s)=0$ for large values of $s$ and $\theta(0)=0$, we have

$$
\begin{aligned}
\int_{I} \frac{\mathrm{d}}{\mathrm{d} t} \int_{\Omega_{\xi(t)}} \theta\left(\Xi^{m}\right) \psi \mathrm{d} \mathbf{x} \mathrm{d} t-\int_{I \times \Omega_{\xi(t)}} \theta\left(\Xi^{m}\right) \partial_{t} \psi \mathrm{d} \mathbf{x} \mathrm{d} t \\
=-\int_{I \times \Omega_{\xi(t)}}\left(\varrho \theta^{\prime}\left(\Xi^{m}\right)-\theta\left(\Xi^{m}\right)\right) \operatorname{div}_{\mathbf{x}} \mathbf{v} \psi \mathrm{d} \mathbf{x} \mathrm{d} t+\int_{I \times \Omega_{\xi(t)}} \theta\left(\Xi^{m}\right) \mathbf{v} \cdot \nabla_{\mathbf{x}} \psi \mathrm{d} \mathbf{x} \mathrm{d} t \\
\quad-\int_{I \times \Omega_{\xi(t)}} \varepsilon \nabla_{\mathbf{x}} \theta\left(\Xi^{m}\right) \cdot \nabla_{\mathbf{x}} \psi \mathrm{d} \mathbf{x} \mathrm{d} t \\
\quad-\int_{I \times \Omega_{\xi(t)}} \varepsilon \theta^{\prime \prime}\left(\Xi^{m}\right)\left|\nabla_{\mathbf{x}} \Xi^{m}\right|^{2} \psi \mathrm{d} \mathbf{x} \mathrm{d} t
\end{aligned}
$$

for all $\psi \in C^{\infty}\left(\bar{I} \times \mathbb{R}^{3}\right)$. In fact, the assumption that the function $\theta$ in (4.35) has linear growth can be replaced by a quadratic growth assumption. Due to (4.34) and a 
smooth approximation argument, we can allow smooth non-negative functions with $\theta(0)=0$ which have linear growth and bounded derivatives. Choosing $\psi$ as a smooth approximation of $\mathbb{I}_{(0, t)}$ and noticing that in our case $\operatorname{div}_{\mathbf{x}} \mathbf{v}=0$, we obtain

$$
\int_{\Omega_{\xi(t)}} \theta\left(\Xi^{m}(t)\right) \mathrm{d} \mathbf{x} \leq \int_{\Omega_{\xi(0)}} \theta\left(\Xi_{0}^{m}\right) \mathrm{d} \mathbf{x}
$$

for all $t \in I$ provided that $\theta$ additionally satisfies $\theta^{\prime \prime} \geq 0$. We would like to choose $\theta(s)=s^{p}$ for large $p$, which is currently not possible. However, we can approximate it by a sequence of convex $C^{2}$-functions $\theta_{N}$ with $\theta_{N}(s)=s^{p}$ for $s \leq N$ and $\theta_{N} \leq c p^{2} \theta$ for all $N \gg 1$. This yields

$$
\int_{\Omega_{\xi(t)}} \theta_{N}\left(\Xi^{m}(t)\right) \mathrm{d} \mathbf{x} \leq \int_{\Omega_{\xi(0)}} \theta\left(\Xi_{0}^{m}\right) \mathrm{d} \mathbf{x} \leq c p^{2} \int_{\Omega_{\xi(0)}}\left|\Xi_{0}^{m}\right|^{p} \mathrm{~d} \mathbf{x}
$$

Fatou's lemma yields $\Xi^{m}(t) \in L^{p}\left(\Omega_{\xi(t)}\right)$ and

$$
\int_{\Omega_{\xi(t)}}\left|\Xi^{m}(t)\right|^{p} \mathrm{~d} \mathbf{x} \leq c p^{2} \int_{\Omega_{\xi(0)}}\left|\Xi_{0}^{m}\right|^{p} \mathrm{~d} \mathbf{x}
$$

Taking the $p$ th root and passing with $p \rightarrow \infty$ implies

$$
\left\|\Xi^{m}(t)\right\|_{L^{\infty}(\Omega(t))} \leq\left\|\Xi_{0}^{m}\right\|_{L^{\infty}\left(\Omega_{\xi(0)}\right)} .
$$

for all $t \in I$. Recalling (4.34), we conclude that

$$
\left\|\Xi^{m}\right\|_{L^{\infty}\left(I \times \Omega_{\xi(t)}\right)}^{2}+\left\|\nabla_{\mathbf{x}} \Xi^{m}\right\|_{L^{2}\left(I \times \Omega_{\xi(t)}\right)}^{2} \lesssim\left\|\Xi_{0}\right\|_{L^{\infty}\left(\Omega_{\xi(0)}\right)}^{2} .
$$

Standard arguments making use of the linearity of (4.31) yield a limit function $\Xi$, as $m \rightarrow \infty$, also satisfying (4.31) and (4.36). We now show that the leading term in the extra stress tensor is essentially bounded too.

Corollary 4.4 For each $i=1, \ldots, K$, let

$$
\mathbb{T}_{i}^{\ell}\left(M \widehat{\psi}^{m}\right):=\int_{B} M(\mathbf{q}) \nabla_{\mathbf{q}_{i}} T_{\ell}\left[\widehat{\psi}^{m}(t, \mathbf{x}, \mathbf{q})\right] \otimes \mathbf{q}_{i} \mathrm{~d} \mathbf{q}
$$

Then for each $i=1, \ldots, K$,

$$
\left|\mathbb{T}_{i}^{\ell}\left(M \widehat{\psi}^{m}\right)\right| \lesssim \ell
$$

uniformly in $m \in \mathbb{N}$.

For the proof of the Corollary above, integrate by parts and use the property of the cut-off $T_{\ell}$, see Bulíček et al. (2013, (2.65)). 


\subsection{Estimates for the Relative Entropy and Fisher Information}

Next, we show an estimate for the relative entropy defined in (2.4) and the Fisher information (2.9) which in our context is given by the second moment of the gradient of the square-root of our solution. Note that a form of energy estimate can be derived by passing to the limit in (4.16). However, this gives no information on the relative entropy which is crucial once we couple the Fokker-Planck equation with the fluid system.

Now, let us recall the entropy functional (2.3) and its convex regularization $\mathcal{F}_{\delta}(\cdot)$ introduced at the start of Sect. 3. If we use $\mathcal{F}_{\delta}^{\prime}\left(\widehat{\psi}^{m}\right)$ as a test-function in (4.25) (justified through (4.26)), then similar to (3.18), we obtain

$$
\begin{aligned}
& \int_{\Omega_{\xi(t)} \times B} k M^{m} \mathcal{F}_{\delta}\left(\widehat{\psi}^{m}\right) \mathrm{d} \mathbf{q} \mathrm{d} \mathbf{x}+k \varepsilon \int_{0}^{t} \int_{\Omega_{\xi(\sigma)} \times B} M^{m}\left|\nabla_{\mathbf{x}} \widehat{\psi}^{m}\right|^{2}\left(\widehat{\psi}^{m}+\delta\right)^{-1} \mathrm{~d} \mathbf{q} \mathrm{d} \mathbf{x} \mathrm{d} \sigma \\
& \quad+\frac{k A_{0}}{4 \lambda} \int_{0}^{t} \int_{\Omega_{\xi(\sigma)} \times B} M^{m}\left|\nabla_{\mathbf{q}} \widehat{\psi}^{m}\right|^{2}\left(\widehat{\psi}^{m}+\delta\right)^{-1} \mathrm{~d} \mathbf{q} \mathrm{d} \mathbf{x} \mathrm{d} \sigma \\
& \quad \leq \int_{\Omega_{\xi(0)} \times B} k M^{m} \mathcal{F}_{\delta}\left(\widehat{\psi}_{0}^{m}\right) \mathrm{d} \mathbf{q} \mathrm{d} \mathbf{x}+k \int_{0}^{t} \int_{\Omega_{\xi(\sigma)} \times B} \sum_{i=1}^{K} M T_{\delta, \ell}\left(\widehat{\psi}^{m}\right) U^{\prime} \\
& \left(\frac{1}{2}\left|\mathbf{q}_{i}\right|^{2}\right) \mathbf{q}_{i} \otimes \mathbf{q}_{i}: \nabla_{\mathbf{x}} \mathbf{v} \mathrm{d} \mathbf{q} \mathrm{d} \mathbf{x} \mathrm{d} \sigma
\end{aligned}
$$

uniformly in $m, \ell \in \mathbb{N}$ and $\delta>0$. By passing to the limit $\delta \rightarrow 0$ (keeping in mind (3.15)), we obtain just as in (3.22),

$$
\begin{aligned}
& \int_{\Omega_{\xi(t)} \times B} k M^{m} \mathcal{F}\left(\widehat{\psi}^{m}(t, \cdot)\right) \mathrm{d} \mathbf{q} \mathrm{d} \mathbf{x} \\
& +4 k \varepsilon \int_{0}^{t} \int_{\Omega_{\xi(\sigma)} \times B} M^{m}\left|\nabla_{\mathbf{x}} \sqrt{\widehat{\psi}^{m}}\right|^{2} \mathrm{~d} \mathbf{q} \mathrm{d} \mathbf{x} \mathrm{d} \sigma \\
& \quad+\frac{k A_{0}}{\lambda} \int_{0}^{t} \int_{\Omega_{\xi(\sigma)} \times B} M^{m}\left|\nabla_{\mathbf{q}} \sqrt{\widehat{\psi}^{m}}\right|^{2} \mathrm{~d} \mathbf{q} \mathrm{d} \mathbf{x} \mathrm{d} \sigma \\
& \leq \int_{\Omega_{\xi(0)} \times B} k M^{m} \mathcal{F}\left(\widehat{\psi}_{0}^{m}\right) \mathrm{d} \mathbf{q} \mathrm{d} \mathbf{x} \\
& \quad+\int_{0}^{t} \int_{\Omega_{\xi(\sigma)}} \mathbb{T}_{\ell}\left(M \widehat{\psi}^{m}\right): \nabla_{\mathbf{x}} \mathbf{v} \mathrm{d} \mathbf{x} \mathrm{d} \sigma .
\end{aligned}
$$

for all $t \in \bar{I}$ where

$$
\begin{aligned}
\mathbb{T}_{\ell}\left(M \widehat{\psi}^{m}\right): & =k \sum_{i=1}^{K} \int_{B} M(\mathbf{q}) \nabla_{\mathbf{q}_{i}} T_{\ell}\left[\widehat{\psi}^{m}(t, \mathbf{x}, \mathbf{q})\right] \otimes \mathbf{q}_{i} \mathrm{~d} \mathbf{q} \\
& -k \Xi^{m} \mathbb{I}-\partial\left(\Xi^{m}\right)^{2} \mathbb{I} .
\end{aligned}
$$




\subsection{Second Layer Approximate Weak Solution}

If we now recall the fact that $\mathbf{v}$ satisfies (4.1), we can use (4.36) and Corollary 4.4 to conclude that the right-hand side of (4.40) is bounded uniformly in $m \in \mathbb{N}$. We show now how to identify the limit, as $m \rightarrow \infty$, of the last term in (4.40). To do this, we first note that there exist a constant $s_{0}>0$ such that

$$
s \ln (\mathrm{e}+s) \lesssim M \mathcal{F}(s / M) \text { if } s \geq s_{0} \text { and } M<c .
$$

Now, we let $\psi^{m}:=M^{m} \widehat{\psi}^{m}$. Since $M^{m}<c$ uniformly in $m \in \mathbb{N}$, for all $t \in I$, we have for some $s_{0}>0$,

$$
\begin{aligned}
\int_{\mathbb{R}^{3} \times B} \chi_{\Omega_{\xi(t)}} \psi^{m} \ln \left(\mathrm{e}+\psi^{m}\right) \mathrm{d} \mathbf{q} \mathrm{d} \mathbf{x}=\int_{\psi^{m}<s_{0}} \chi \Omega_{\xi(t)} \psi^{m} \ln \left(\mathrm{e}+\psi^{m}\right) \mathrm{d} \mathbf{q} \mathrm{d} \mathbf{x} \\
\quad+\int_{\psi^{m} \geq s_{0}} \chi_{\Omega_{\xi(t)}} \psi^{m} \ln \left(\mathrm{e}+\psi^{m}\right) \mathrm{d} \mathbf{q} \mathrm{d} \mathbf{x} \lesssim 1+\int_{\mathbb{R}^{3} \times B} \chi_{\Omega_{\xi(t)}} M^{m} \mathcal{F}\left(\widehat{\psi}^{m}\right) \mathrm{d} \mathbf{q} \mathrm{d} \mathbf{x}
\end{aligned}
$$

with a constant depending only on the Lebesgue measure of $\Omega_{\xi(t)} \times B$ and $s_{0}$. By using (4.40), we can conclude that

$$
\sup _{t \in I} \int_{\mathbb{R}^{3} \times B} \chi_{\Omega_{\xi(t)}} \psi^{m} \ln \left(\mathrm{e}+\psi^{m}\right) \mathrm{d} \mathbf{q} \mathrm{d} \mathbf{x} \lesssim 1
$$

holds uniformly in $m \in \mathbb{N}$ and thus, $\psi^{m}$ is equi-integrable. In order to obtain compactness for $\widehat{\psi}^{m}$, we have to localise the equation to avoid problems with the moving boundary. We consider a sequence $\mathcal{Q}_{k}=J^{k} \times \mathcal{B}_{\mathbf{x}}^{k} \times \mathcal{B}_{\mathbf{q}}^{k}$ (the $\mathcal{B}_{\mathbf{x}}^{k} \mathrm{~s}$ and $\mathcal{B}_{\mathbf{q}}^{k}$ s are open balls and the $J_{k}$ s open intervals), $k \in \mathbb{N}$ such that $\bigcup_{k} \mathcal{Q}_{k}=I \times \Omega_{\xi} \times B J_{k} \Subset I$, $\mathcal{B}_{\mathbf{x}}^{k} \Subset \bigcup_{t \in J_{k}} \Omega_{\xi(t)}$ and $\mathcal{B}_{\mathbf{q}}^{k} \Subset B$ for all $k \in \mathbb{N}$. Now fixed $k \in \mathbb{N}$ and use $\varphi \in C_{c}^{\infty}\left(\mathcal{Q}_{k}\right)$ as a test-function in (4.23) to conclude

$$
\partial_{t} \widehat{\psi}^{m} \in L^{2}\left(J_{k} ; W^{-1,2}\left(\mathcal{B}_{\mathbf{x}}^{k} \times \mathcal{B}_{\mathbf{q}}^{k}\right)\right)
$$

uniformly in $m$ using the uniform bounds from (4.40). Since $M^{m}$ is strictly positive in $\mathcal{B}_{\mathbf{q}}^{k}$ with a bound depending on $k$ but independent of $m$, we also have

$$
\widehat{\psi}^{m} \in L^{2}\left(J_{k} ; W^{1,2}\left(\mathcal{B}_{\mathbf{x}}^{k} \times \mathcal{B}_{\mathbf{q}}^{k}\right)\right)
$$

uniformly. Consequently, we obtain

$$
\widehat{\psi}^{m} \rightarrow \widehat{\psi} \quad \text { in } L^{2}\left(\mathcal{Q}_{k}\right)
$$

for a subsequence as well as

$$
\chi_{\Omega_{\xi(t)}} \widehat{\psi}^{m} \rightarrow \chi_{\Omega_{\xi(t)}} \widehat{\psi} \text { a.e. in } I \times \mathbb{R}^{3} \times B
$$


by taking a diagonal sequence. Due to uniform convergence of $M^{m}$ to $M$, cf. (4.11), we also obtain

$$
\chi_{\Omega_{\xi(t)}} \psi^{m} \rightarrow \chi_{\Omega_{\xi(t)}} \psi \text { a.e. in } I \times \mathbb{R}^{3} \times B
$$

By combining (4.44) with (4.42), we can conclude from Vitali's convergence theorem that

$$
\psi^{m} \rightarrow \psi \quad \text { in } \quad L^{1}\left(I \times \Omega_{\xi(t)} \times B\right)
$$

and by interpolation with (4.36),

$$
\psi^{m} \rightarrow \psi \quad \text { in } \quad L^{q}\left(I \times \Omega_{\xi(t)} ; L^{1}(B)\right) \text { for all } q \in[1, \infty) .
$$

This is enough to pass to the limit $m \rightarrow \infty$ in (4.40) so that together with results from Sect. 4.3, we obtain

$$
\begin{aligned}
& \|\Xi(t, \cdot)\|_{L^{\infty}\left(\Omega_{\xi(t)}\right)}^{2}+k \int_{\Omega_{\xi(t)} \times B} M \mathcal{F}(\widehat{\psi}(t, \cdot, \cdot)) \mathrm{d} \mathbf{q} \mathrm{d} \mathbf{x}+\varepsilon \int_{0}^{t} \int_{\Omega_{\xi(\sigma)}}\left|\nabla_{\mathbf{x}} \Xi\right|^{2} \mathrm{~d} \mathbf{x} \mathrm{d} \sigma \\
& +4 k \varepsilon \int_{0}^{t} \int_{\Omega_{\xi(\sigma)} \times B} M\left|\nabla_{\mathbf{x}} \sqrt{\widehat{\psi}}\right|^{2} \mathrm{~d} \mathbf{q} \mathrm{d} \mathbf{x} \mathrm{d} \sigma+\frac{k A_{0}}{\lambda} \int_{0}^{t} \int_{\Omega_{\xi(\sigma)} \times B} M \mid \nabla_{\mathbf{q}} \sqrt{\widehat{\psi}}^{2} \mathrm{~d} \mathbf{q} \mathrm{d} \mathbf{x} \mathrm{d} \sigma \\
& \lesssim\left\|\Xi_{0}\right\|_{L^{\infty}\left(\Omega_{\xi(0)}\right)}^{2}+k \int_{\Omega_{\xi(0) \times B}} M \mathcal{F}\left(\widehat{\psi}_{0}\right) \mathrm{d} \mathbf{q} \mathrm{d} \mathbf{x} \\
& +\int_{0}^{t} \int_{\Omega_{\xi(\sigma)}} \mathbb{T}_{\ell}(M \widehat{\psi}): \nabla_{\mathbf{x}} \mathbf{v} \mathrm{d} \mathbf{x} \mathrm{d} \sigma .
\end{aligned}
$$

for all $t \in I$. To finally identify the distributional solution solved by the limit $\widehat{\psi}:=\widehat{\psi}^{\ell}$, we first note that for all $j=1, \ldots, K$, the following convergence

$$
\begin{array}{ccc}
M^{m} \nabla_{\mathbf{x}} \widehat{\psi}^{m}-M \nabla_{\mathbf{x}} \widehat{\psi} & \text { in } & L^{2}\left(I \times \Omega_{\xi(t)} ; L^{1}\left(B ; \mathbb{R}^{3}\right)\right) \\
M^{m} \nabla_{\mathbf{q}_{j}} \widehat{\psi}^{m} \rightarrow M \nabla_{\mathbf{q}_{j}} \widehat{\psi} & \text { in } \quad L^{2}\left(I \times \Omega_{\xi(t)} ; L^{1}\left(B ; \mathbb{R}^{3 K}\right)\right)
\end{array}
$$

holds as $m \rightarrow \infty$. To avoid repetition, we refer the reader to Bulíček et al. (2013, (2.100)). Using (4.47)-(4.48), the uniform convergence of $M^{m}$ as well as (4.45), we can pass to the limit in (4.23) to obtain

$$
\begin{aligned}
& \int_{I} \frac{\mathrm{d}}{\mathrm{d} t} \int_{\Omega_{\xi(t)} \times B} M \widehat{\psi} \varphi \mathrm{d} \mathbf{q} \mathrm{d} \mathbf{x} \mathrm{d} t=\int_{I \times \Omega_{\xi(t)} \times B} M \widehat{\psi} \partial_{t} \varphi \mathrm{d} \mathbf{q} \mathrm{d} \mathbf{x} \mathrm{d} t \\
& \quad+\int_{I \times \Omega_{\xi(t)} \times B}\left(\mathbf{v} M \widehat{\psi}-\varepsilon M \nabla_{\mathbf{x}} \widehat{\psi}\right) \cdot \nabla_{\mathbf{x}} \varphi \mathrm{d} \mathbf{q} \mathrm{d} \mathbf{x} \mathrm{d} t \\
& \quad+\sum_{i=1}^{K} \int_{I \times \Omega_{\xi(t)} \times B}\left(M\left(\nabla_{\mathbf{x}} \mathbf{v}\right) \mathbf{q}_{i} \Lambda_{\ell}(\widehat{\psi})-\sum_{j=1}^{K} \frac{A_{i j}}{4 \lambda} M \nabla_{\mathbf{q}_{j}} \widehat{\psi}\right) \cdot \nabla_{\mathbf{q}_{i}} \varphi \mathrm{d} \mathbf{q} \mathrm{d} \mathbf{x} \mathrm{d} t
\end{aligned}
$$


for all $\varphi \in C^{\infty}\left(\bar{I} \times \mathbb{R}^{3} \times \bar{B}\right)$.

\subsection{Conclusion}

By recalling Remark 4.3, we note that $\widehat{\psi}:=\widehat{\psi}^{\ell}$ where $\ell \in \mathbb{N}$ is fixed and $\psi=M \widehat{\psi}:=$ $M \widehat{\psi}^{\ell}$. Since (4.46) holds independently of $\ell \in \mathbb{N}$, just as in (4.42), we obtain

$$
\sup _{t \in I} \int_{\Omega_{\xi(t)} \times B} \psi^{\ell} \ln \left(\mathrm{e}+\psi^{\ell}\right) \mathrm{d} \mathbf{q} \mathrm{d} \mathbf{x} \lesssim 1
$$

uniformly in $\ell \in \mathbb{N}$ from which we obtain

$$
\psi^{\ell} \rightarrow \psi \quad \text { in } \quad L^{q}\left(I \times \Omega_{\xi(t)} ; L^{1}(B)\right) \text { for all } q \in[1, \infty)
$$

exactly as in (4.45). Using (4.51) and the definition of $\mathbb{T}_{\ell}$ given by (4.41), we can in particular, pass to the limit $\ell \rightarrow \infty$ in the last term in (4.46). Subsequently, we obtain (4.9) for all $t \in I$. Similar to (4.49), we also obtain (4.8) for all $\varphi \in C^{\infty}\left(\bar{I} \times \mathbb{R}^{3} \times \bar{B}\right)$ by using (4.51). This completes the proof of Theorem 4.2 .

\section{The Regularized System}

Let $\varrho>0$ be a fixed regularization kernel. Our ultimate goal in this section is to construct on $I \times \Omega_{\mathcal{R}^{\varrho} \eta^{\varrho}} \times B$, a weak solution to a version of our fluid-structurekinetic system transported by a regularized material derivative

$$
\partial_{t}+\mathcal{R}^{\varrho} \mathbf{u}^{\varrho} \cdot \nabla_{\mathbf{x}}
$$

similar to Lengeler and Růžička (2014). Here, $\left(\mathcal{R}^{\varrho}\right)_{\varrho>0}$ is a family of regularization operators defined as the composition of a temporal regularisation on $I$ (which is symmetric and commutes with derivatives) and a mollification by a smooth kernel. For the later one to make sense we extend the corresponding functions by zero to the whole space. In (5.1) $\eta^{\varrho}$ and $\mathbf{u}^{\varrho}$ are corresponding approximate solutions of the shell equation (1.7) and momentum equation (1.21) respectively. We now recall that unlike (Lengeler and Růžička 2014) where a linearized Koiter elastic energy is considered, we are working with the more realistic fully nonlinear energy. To be able to proceed therefore, additionally, we regularize the shell equation by the higher order linear term $\varrho \mathcal{L}^{\prime}(\eta)$ where $\mathcal{L}(\eta)=\int_{\omega}\left|\nabla_{\mathbf{y}}^{5} \eta\right|^{2} \mathrm{~d} \mathbf{y}$ (which is to be interpreted in the sense that $\int_{\omega} \varrho \mathcal{L}^{\prime}(\eta) \phi \mathrm{d} \mathbf{y}=\int_{\omega} \varrho \nabla_{\mathbf{y}}^{5} \eta: \nabla_{\mathbf{y}}^{5} \phi \mathrm{d} \mathbf{y}$ for all $\left.\phi \in W^{5,2}(\omega)\right)$. Once the above construction is done, we can pass to the limit $\varrho \rightarrow 0$ to complete the proof of our main result, Theorem 2.6. This will be done in the next section.

Besides regularizing the shell equation by $\varrho \mathcal{L}^{\prime}(\eta)$ as earlier explained, we require further, two main tools to achieve our goal for this section: a Galerkin procedure and a fixed-point argument. Unfortunately, we are required to have a fully linear system and require an additional regularization procedure in order to apply our choice of 
fixed-point argument. These two obstacles and their remedies leads to the following steps in achieving our goal:

The following steps give the line-by-line reasoning as to why we require (5.1).

(1) First of all, we note that one source of nonlinearity in our system comesfrom the convective term in the momentum equation (1.21). Furthermore, the spatial domain on which a solution to our full system is defined depends on the solution itself which is problematic. We remedy these problems using the following.

(a) We linearise the transport term of the momentum equation by replacing the material derivation $\partial_{t}+\mathbf{u} \cdot \nabla_{\mathbf{x}}$ with $\partial_{t}+\mathbf{v} \cdot \nabla_{\mathbf{x}}$ where $\mathbf{v}$ is a given velocity field. In addition, we replace the spatial domain with $\Omega_{\xi}$ where $\xi$ is a given function of time.

(b) Because of the highly coupled nature of our fluid-structure-kinetic system, we are also required to replace the transport term of the Fokker-Planck equation with $\partial_{t}+\mathbf{v} \cdot \nabla_{\mathbf{x}}$. This makes physically sense since one can expect the transport of the solute to be affected by a change in transport of the solvent in the solution. Finally, we linearise the shell equation by replacing $K^{\prime}(\eta)$ by $K^{\prime}(\xi)$, which will be considered as part of the right-hand side.

(2) At this point, in theory, we should be able to apply a Gelerkin method to show that a solution to our modified-transport problem exists. Unfortunately, we are constrained by the low regularity of the boundary. For this reason, we construct instead, a solution to our system on $\Omega_{\mathfrak{r}} \varrho \xi \varrho$ with material derivation $\partial_{t}+\mathcal{R}^{\varrho} \mathbf{v}^{\varrho} \cdot \nabla_{\mathbf{x}}$ where $\varrho>0$ is a fixed regularization kernel. Here $\mathfrak{r}^{\varrho}$ is regularisation operator acting on the periodic functions defined on $\omega$ composed again with a temporal regularisation on $I$.

(3) Finally, for $\varrho>0$ fixed, we can use a Schauder-type fixed-point argument to the mapping $\left(\xi^{\varrho}, \mathbf{v}^{\varrho}\right) \mapsto\left(\eta^{\varrho}, \mathbf{u}^{\varrho}\right)$ where $\eta^{\varrho}$ and $\mathbf{u}^{\varrho}$ are solutions to the decoupled regularised shell equation with data $\left(\xi^{\varrho}, \mathbf{v}^{\varrho}\right)$ to be defined below in Sect. 5.1.

Henceforth, we simply write $(\xi, \mathbf{v})$ in place of $\left(\xi^{\varrho}, \mathbf{v}^{\varrho}\right)_{\varrho>0}$ (and the same for $\left.(\eta, \mathbf{u})\right)$ until the next section when we pass to the limit $\varrho \rightarrow 0$. However, to emphasize that our regularization is parametrized by $\varrho>0$, we maintain the notation $\mathcal{R} \varrho$ in this chapter.

With the above introduction, we now make precise, our goal of this section.

We now seek to find $(\mathbf{u}, \widehat{\psi}, \eta):=\left(\mathbf{u}^{\varrho}, \widehat{\psi}^{\varrho}, \eta^{\varrho}\right)$ that solves the following system

$$
\begin{aligned}
& \operatorname{div}_{\mathbf{x}} \mathbf{u}=0 \\
& \partial_{t}^{2} \eta+K^{\prime}(\eta)+\varrho \mathcal{L}^{\prime}(\eta)=g+\mathbf{F} \cdot \mathbf{v}, \\
& \partial_{t} \mathbf{u}+\left(\mathcal{R}^{\varrho} \mathbf{u} \cdot \nabla_{\mathbf{x}}\right) \mathbf{u}+\nabla_{\mathbf{x}} p=\mu \Delta_{\mathbf{x}} \mathbf{u}+\mathcal{R}^{\varrho} \operatorname{div}_{\mathbf{x}} \mathbb{T}(M \widehat{\psi})+\mathbf{f} \\
& \partial_{t}(M \widehat{\psi})+\left(\mathcal{R}^{\varrho} \mathbf{u} \cdot \nabla_{\mathbf{x}}\right) M \widehat{\psi}=\varepsilon \Delta_{\mathbf{x}}(M \widehat{\psi})-\sum_{i=1}^{K} \operatorname{div}_{\mathbf{q}_{i}}\left(M\left(\nabla_{\mathbf{x}} \mathcal{R}^{\varrho} \mathbf{u}\right) \mathbf{q}_{i} \widehat{\psi}\right) \\
& +\frac{1}{4 \lambda} \sum_{i=1}^{K} \sum_{j=1}^{K} A_{i j} \operatorname{div}_{\mathbf{q}_{i}}\left(M \nabla_{\mathbf{q}_{j}} \widehat{\psi}\right)
\end{aligned}
$$


in $I \times \Omega_{\mathfrak{r}} \eta_{\eta(t)} \times B$ subject to the following initial and boundary conditions

$$
\begin{array}{lr}
\mathbf{u}(0, \cdot)=\mathbf{u}_{0} & \text { in } \Omega_{\mathfrak{r}} \varrho \eta_{0}, \\
\eta(0, \cdot)=\eta_{0}, \quad \partial_{t} \eta(0, \cdot)=\eta_{1} & \text { in } \omega, \\
\mathbf{u}\left(t, \varphi(\mathbf{y})+\mathfrak{r}^{\varrho} \eta(t, \mathbf{y}) \mathbf{v}(\mathbf{y})\right)=\partial_{t} \eta(t, \mathbf{y}) \mathbf{v}(\mathbf{y}) & \text { on } I \times \omega
\end{array}
$$

and for $\mathrm{i}=1, \ldots, \mathrm{K}$,

$$
\begin{aligned}
& {\left[\frac{1}{4 \lambda} \sum_{j=1}^{K} A_{i j} M \nabla_{\mathbf{q}_{j}} \widehat{\psi}-M\left(\nabla_{\mathbf{x}} \mathcal{R}^{\varrho} \mathbf{u}\right) \mathbf{q}_{i} \widehat{\psi}\right] \cdot \mathbf{n}_{i}=0 \quad \text { on } I \times \Omega_{\mathfrak{r}^{\varrho} \eta(t)} \times \partial \bar{B}_{i},} \\
& \nabla_{\mathbf{y}} \widehat{\psi} \cdot \boldsymbol{v}_{\mathfrak{r}}{ } \eta=\widehat{\psi}\left(\mathcal{R}^{\varrho} \mathbf{u}-\left(\partial_{t} \mathfrak{r}^{\varrho} \eta \boldsymbol{v}\right) \circ \varphi_{\mathfrak{r}^{\varrho} \eta}^{-1}\right) \cdot \boldsymbol{v}_{\mathfrak{r}} \varrho_{\eta} \quad \text { on } I \times \partial \Omega_{\mathfrak{r} \varrho} \eta(t) \times B, \\
& \widehat{\psi}(0, \cdot, \cdot)=\widehat{\psi}_{0} \geq 0 \quad \text { in } \Omega_{\mathrm{r} e} \eta_{0} \times B,
\end{aligned}
$$

where $\eta_{0}, \eta_{1}: \omega \rightarrow \mathbb{R}$ and $g: I \times \omega \rightarrow \mathbb{R}$ are given functions and

$\mathbf{F}(t, \mathbf{y}):=\left(-2 \mu \mathbb{D}_{\mathbf{y}} \mathbf{u}(t, \mathbf{y})-\mathcal{R}^{\varrho} \mathbb{T}(M \widehat{\psi})(t, \mathbf{y})+p(t, \mathbf{y}) \mathbb{I}\right) \boldsymbol{v} \circ \boldsymbol{\varphi}_{\mathfrak{r} \varrho \eta(t)}\left|\operatorname{det} D \boldsymbol{\varphi}_{\mathfrak{r} \varrho \eta(t)}\right|$

Let us start with a precise definition of the solution. Note that as in Lengeler and Růžička (2014) we have to rewrite the convective term in an uncommon way to preserve the a priori estimates.

Definition 5.1 (Finite energy weak solution) Let (f), $\left.g, \eta_{0}, \widehat{\psi}_{0}, \mathbf{u}_{0}, \eta_{1}\right)$ be a dataset such that

$$
\begin{aligned}
& \mathbf{f} \in L^{2}\left(I ; L_{\mathrm{loc}}^{2}\left(\mathbb{R}^{3} ; \mathbb{R}^{3}\right)\right), \quad g \in L^{2}\left(I ; L^{2}(\omega)\right), \quad \eta_{0} \in W^{5,2}(\omega) \text { with }\left\|\eta_{0}\right\|_{L^{\infty}(\omega)}<L, \\
& \widehat{\psi}_{0} \in L^{2}\left(\Omega_{\eta_{0}} ; L_{M}^{2}(B)\right), \quad \mathbf{u}_{0} \in L_{\mathrm{div}_{\mathbf{x}}}^{2}\left(\Omega_{\eta_{0}} ; \mathbb{R}^{3}\right) \text { is such that } \\
& \operatorname{tr}_{\eta_{0}} \mathbf{u}_{0}=\eta_{1} \gamma\left(\eta_{0}\right), \quad \eta_{1} \in L^{2}(\omega) .
\end{aligned}
$$

In addition, we assume

$$
\Xi_{0} \in L^{\infty}\left(\Omega_{\eta_{0}}\right) \text { where } \Xi_{0}=\int_{B} M(\mathbf{q}) \widehat{\psi}_{0}(\cdot, \mathbf{q}) \mathrm{d} \mathbf{q} \text { in } \Omega_{\eta_{0}}
$$

We call the triple $(\mathbf{u}, \widehat{\psi}, \eta)$ a finite energy weak solution to the system (5.2)-(5.12) with data (f) $\left.g, \eta_{0}, \widehat{\psi}_{0}, \mathbf{u}_{0}, \eta_{1}\right)$ provided that the following holds:

(a) the velocity $\mathbf{u}$ satisfies

$$
\mathbf{u} \in L^{\infty}\left(I ; L^{2}\left(\Omega_{\mathfrak{r} \varrho} \eta(t) ; \mathbb{R}^{3}\right)\right) \cap L^{2}\left(I ; W_{\operatorname{div}_{\mathbf{x}}}^{1,2}\left(\Omega_{\mathfrak{r} \varrho} \eta(t) ; \mathbb{R}^{3}\right)\right) \text { and }
$$

in the sense of traces and $\eta$ satisfies

$$
\eta \in W^{1, \infty}\left(I ; L^{2}(\omega)\right) \cap L^{\infty}\left(I ; W^{5,2}(\omega)\right) \text { with }\|\eta\|_{L^{\infty}(I \times \omega)}<L
$$


and for all $(\phi, \varphi) \in C^{\infty}(\bar{I} \times \omega) \times C^{\infty}\left(\bar{I} \times \mathbb{R}^{3} ; \mathbb{R}^{3}\right)$ with $\phi(T, \cdot)=0, \varphi(T, \cdot)=0$, $\operatorname{div}_{\mathbf{x}} \varphi=0$ and $\operatorname{tr}_{\mathfrak{r}}{ }_{\eta} \varphi=\phi v$, we have

$$
\begin{aligned}
\int_{I} & \frac{\mathrm{d}}{\mathrm{d} t}\left(\int_{\Omega_{\mathfrak{r}} \varrho_{\eta(t)}} \mathbf{u} \cdot \boldsymbol{\varphi} \mathrm{d} \mathbf{x}+\int_{\omega} \partial_{t} \eta \phi \mathrm{d} \mathbf{y}\right) \mathrm{d} t \\
= & \int_{I} \int_{\Omega_{\mathfrak{r}} \varrho_{\eta(t)}}\left(\mathbf{u} \cdot \partial_{t} \boldsymbol{\varphi}-\frac{1}{2}\left(\mathcal{R}^{\varrho} \mathbf{u} \cdot \nabla\right) \mathbf{u} \cdot \boldsymbol{\varphi}+\frac{1}{2}\left(\mathcal{R}^{\varrho} \mathbf{u} \cdot \nabla\right) \boldsymbol{\varphi} \cdot \mathbf{u}\right) \mathrm{d} \mathbf{x} \mathrm{d} t \\
& -\int_{I} \int_{\Omega_{\mathfrak{r}} \varrho_{\eta(t)}}\left(\mu \nabla_{\mathbf{x}} \mathbf{u}: \nabla_{\mathbf{x}} \boldsymbol{\varphi}+\mathcal{R}^{\varrho} \mathbb{T}(M \widehat{\psi}): \nabla_{\mathbf{x}} \boldsymbol{\varphi}-\mathbf{f} \cdot \boldsymbol{\varphi}\right) \mathrm{d} \mathbf{x} \mathrm{d} t \\
& +\int_{I} \int_{\omega}\left(\partial_{t} \eta \partial_{t} \phi+\frac{1}{2} \partial_{t} \eta \partial_{t} \mathfrak{r}^{\varrho} \eta \phi+g \phi\right) \mathrm{d} \mathbf{y} \mathrm{d} t \\
& -\int_{I}\left(\left\langle K^{\prime}(\eta), \phi\right\rangle+\varrho\left\langle\mathcal{L}^{\prime}(\eta), \phi\right\rangle\right) \mathrm{d} t
\end{aligned}
$$

(b) the probability density function $\widehat{\psi}$ satisfies:

$$
\begin{aligned}
& \widehat{\psi} \geq 0 \text { a.e. in } I \times \Omega_{\mathfrak{r} \varrho_{\eta}(t)} \times B, \\
& \widehat{\psi} \in L^{\infty}\left(I \times \Omega_{\mathfrak{r} \varrho} \eta_{(t)} ; L_{M}^{1}(B)\right), \\
& \mathcal{F}(\widehat{\psi}) \in L^{\infty}\left(I ; L^{1}\left(\Omega_{\mathfrak{r} \varrho} \varrho_{(t)} ; L_{M}^{1}(B)\right)\right), \\
& \sqrt{\widehat{\psi}} \in L^{2}\left(I ; L^{2}\left(\Omega_{\mathfrak{r} \varrho} \eta_{(t)} ; W_{M}^{1,2}(B)\right)\right) \cap L^{2}\left(I ; D^{1,2}\left(\Omega_{\mathfrak{r} \varrho} \eta(t) ; L_{M}^{2}(B)\right)\right), \\
& \Xi(t, \mathbf{x})=\int_{B} M \widehat{\psi}(t, \mathbf{x}, \mathbf{q}) \mathrm{d} \mathbf{q} \in L^{\infty}\left(I \times \Omega_{\mathfrak{r} \varrho} \eta(t)\right) \cap L^{2}\left(I ; W^{1,2}\left(\mathfrak{r}^{\varrho} \eta(t)\right)\right) ;
\end{aligned}
$$

and for all $\varphi \in C^{\infty}\left(\bar{I} \times \mathbb{R}^{3} \times \bar{B}\right)$, we have

$$
\begin{aligned}
& \int_{I} \frac{\mathrm{d}}{\mathrm{d} t} \int_{\Omega_{\mathrm{r}} \varrho_{\eta(t)} \times B} M \widehat{\psi} \varphi \mathrm{d} \mathbf{q} \mathrm{d} \mathbf{x} \mathrm{d} t \\
& =\int_{I \times \Omega_{\mathrm{r}} \varrho_{\eta(t)} \times B}\left(M \widehat{\psi} \partial_{t} \varphi+M \mathcal{R}^{\varrho} \mathbf{u} \widehat{\psi} \cdot \nabla_{\mathbf{x}} \varphi-\varepsilon M \nabla_{\mathbf{x}} \widehat{\psi} \cdot \nabla_{\mathbf{x}} \varphi\right) \mathrm{d} \mathbf{q} \mathrm{d} \mathbf{x} \mathrm{d} t \\
& \quad+\sum_{i=1}^{K} \int_{I \times \Omega_{\mathrm{r}} \varrho_{\eta(t)} \times B}\left(M\left(\nabla_{\mathbf{x}} \mathcal{R}^{\varrho} \mathbf{u}\right) \mathbf{q}_{i} \widehat{\psi}-\sum_{j=1}^{K} \frac{A_{i j}}{4 \lambda} M \nabla_{\mathbf{q}_{j}} \widehat{\psi}\right) \cdot \nabla_{\mathbf{q}_{i}} \varphi \mathrm{d} \mathbf{q} \mathrm{d} \mathbf{x} \mathrm{d} t
\end{aligned}
$$

(c) for all $t \in I$, we have

$$
\begin{gathered}
\int_{\Omega_{\mathrm{r} \varrho_{\eta} \varrho_{(t)}}} \frac{1}{2}|\mathbf{u}(t)|^{2} \mathrm{~d} \mathbf{x}+\mu \int_{0}^{t} \int_{\Omega_{\mathrm{r} \varrho_{\eta} \varrho_{(\sigma)}}}\left|\nabla_{\mathbf{x}} \mathbf{u}\right|^{2} \mathrm{~d} \mathbf{x} \mathrm{d} \sigma+\int_{\omega} \frac{1}{2}\left|\partial_{t} \eta(t)\right|^{2} \mathrm{~d} \mathbf{y}+K(\eta(t)) \\
\quad+\varrho \int_{\omega}\left|\nabla_{\mathbf{y}}^{5} \eta(t)\right|^{2} \mathrm{~d} \mathbf{y}+\|\Xi(t, \cdot)\|_{L^{\infty}\left(\Omega_{\mathfrak{r}} \varrho_{\eta} \varrho_{(t)}\right)}^{2}+\varepsilon \int_{0}^{t} \int_{\Omega_{\mathfrak{r} \varrho_{\eta} \varrho_{(\sigma)}}}\left|\nabla_{\mathbf{x}} \Xi\right|^{2} \mathrm{~d} \mathbf{x} \mathrm{d} \sigma \\
\quad+k \int_{\Omega_{\mathfrak{r} \varrho_{\eta} \varrho_{(t)} \times B}} M \mathcal{F}(\widehat{\psi}(t)) \mathrm{d} \mathbf{q} \mathrm{d} \mathbf{x}+4 k \varepsilon \int_{0}^{t} \int_{\Omega_{\mathfrak{r} \varrho_{\eta} \varrho_{(\sigma)} \times B}} M\left|\nabla_{\mathbf{x}} \sqrt{\widehat{\psi}}\right|^{2} \mathrm{~d} \mathbf{q} \mathrm{d} \mathbf{x} \mathrm{d} \sigma
\end{gathered}
$$




$$
\begin{aligned}
& +\frac{k A_{0}}{\lambda} \int_{0}^{t} \int_{\Omega_{\mathfrak{r} \varrho_{\eta} \varrho_{(\sigma)} \times B}} M\left|\nabla_{\mathbf{q}} \sqrt{\widehat{\psi}}\right|^{2} \mathrm{~d} \mathbf{q} \mathrm{d} \mathbf{x} \mathrm{d} \sigma \\
& \lesssim \int_{\Omega_{\mathfrak{r} \varrho} \eta_{0}^{\varrho}} \frac{1}{2}\left|\mathbf{u}_{0}\right|^{2} \mathrm{~d} \mathbf{x}+\int_{\omega} \frac{1}{2}\left|\eta_{1}\right|^{2} \mathrm{~d} \mathbf{y}+K\left(\eta_{0}\right)+\varrho \int_{\omega}\left|\nabla_{\mathbf{y}}^{5} \eta_{0}\right|^{2} \mathrm{~d} \mathbf{y}+\left\|\Xi_{0}\right\|_{L^{\infty}\left(\Omega_{\mathfrak{r}} \eta_{0} \varrho\right)}^{2} \\
& +k \int_{\Omega_{\mathfrak{r} \varrho} \eta_{0}^{\varrho} \times B} M \mathcal{F}\left(\widehat{\psi}_{0}\right)+\frac{1}{2} \int_{0}^{t} \int_{\Omega_{\mathfrak{r}} \varrho_{\eta} \varrho(\sigma)} \mathbf{f} \cdot \mathbf{u} \mathrm{d} \mathbf{x} \mathrm{d} \sigma \\
& +\frac{1}{2} \int_{0}^{t} \int_{\omega} g \partial_{t} \eta \mathrm{d} \mathbf{y} \mathrm{d} \sigma .
\end{aligned}
$$

The following result establishes the existence of a solution, in the sense of Definition 5.1 to the regularized system (5.2)-(5.12), see Remark 2.7 for the case $\lim _{t \rightarrow T}\|\eta(t)\|_{L^{\infty}(\omega)}=L$.

Theorem 5.2 Let (f), $\left.g, \eta_{0}, \widehat{\psi}_{0}, \mathbf{u}_{0}, \eta_{1}\right)$ be a dataset satisfying (5.13) and (5.14). Then there is a finite energy weak solution $(\mathbf{u}, \widehat{\psi}, \eta)$ of the system (5.2)-(5.12) on the interval $I=(0, T)$ in the sense of Definition 5.1. The number $T$ is restricted only if $\lim _{t \rightarrow T}\|\eta(t)\|_{L^{\infty}(\omega)}=L$.

The proof of Theorem 5.2 will be given in Sect. 5.2. It as based on a fixed point argument applied to a linearized system. The latter one will be introduced and analyzed in the next subsection.

\subsection{The Linearised System}

Having constructed a solution $\widehat{\psi}$ to the Fokker-Planck equation in Sect. 4, we now aim to derive a triple $(\mathbf{u}, \widehat{\psi}, \eta)$ that solves the linearized fluid-structure-kinetic system transported by a given velocity $\mathbf{v} \in L_{\operatorname{div}_{\mathbf{x}}}^{2}\left(I \times \mathbb{R}^{3} ; \mathbb{R}^{3}\right)$ on a given shell function $\xi \in C(\bar{I} \times \omega)$, i.e., for a given pair $(\mathbf{v}, \xi)$, we seek to find $(\mathbf{u}, \widehat{\psi}, \eta)$ that solve the following system

$$
\begin{aligned}
& \operatorname{div}_{\mathbf{x}} \mathbf{u}=0, \\
& \partial_{t}^{2} \eta+K^{\prime}(\xi)+\varrho \mathcal{L}^{\prime}(\eta)=g+\mathbf{F} \cdot \mathbf{v} \\
& \partial_{t} \mathbf{u}+\left(\mathcal{R}^{\varrho} \mathbf{v} \cdot \nabla_{\mathbf{x}}\right) \mathbf{u}+\nabla_{\mathbf{x}} p=\mu \Delta_{\mathbf{x}} \mathbf{u}+\mathcal{R}^{\varrho} \operatorname{div}_{\mathbf{x}} \mathbb{T}(M \widehat{\psi})+\mathbf{f}, \\
& \partial_{t}(M \widehat{\psi})+\left(\mathcal{R}^{\varrho} \mathbf{v} \cdot \nabla_{\mathbf{x}}\right) M \widehat{\psi}=\varepsilon \Delta_{\mathbf{x}}(M \widehat{\psi})-\sum_{i=1}^{K} \operatorname{div}_{\mathbf{q}_{i}}\left(M\left(\nabla_{\mathbf{x}} \mathcal{R}^{\varrho} \mathbf{v}\right) \mathbf{q}_{i} \widehat{\psi}\right) \\
& +\frac{1}{4 \lambda} \sum_{i=1}^{K} \sum_{j=1}^{K} A_{i j} \operatorname{div}_{\mathbf{q}_{i}}\left(M \nabla_{\mathbf{q}_{j}} \widehat{\psi}\right)
\end{aligned}
$$

in $I \times \Omega_{\mathfrak{r} \varrho \xi(t)} \times B$ subject to the following initial and boundary conditions

$$
\begin{array}{lr}
\mathbf{u}(0, \cdot)=\mathbf{u}_{0} & \text { in } \Omega_{\mathfrak{r}} \varrho \xi_{0}, \\
\eta(0, \cdot)=\eta_{0}, & \partial_{t} \eta(0, \cdot)=\eta_{1}
\end{array}
$$




$$
\mathbf{u}\left(t, \varphi(\mathbf{y})+\mathfrak{r}^{\varrho} \xi(t, \mathbf{y}) \boldsymbol{v}(\mathbf{y})\right)=\partial_{t} \eta(t, \mathbf{y}) \boldsymbol{v}(\mathbf{y}) \quad \text { on } I \times \omega
$$

and for $\mathrm{i}=1, \ldots, \mathrm{K}$,

$$
\begin{array}{ll}
{\left[\frac{1}{4 \lambda} \sum_{j=1}^{K} A_{i j} M \nabla_{\mathbf{q}_{j}} \widehat{\psi}-M\left(\nabla_{\mathbf{x}} \mathcal{R}^{\varrho} \mathbf{v}\right) \mathbf{q}_{i} \widehat{\psi}\right] \cdot \mathbf{n}_{i}=0} & \text { on } I \times \Omega_{\mathfrak{r}^{\varrho} \xi(t)} \times \partial \bar{B}_{i}, \\
\varepsilon \nabla_{\mathbf{y}} \widehat{\psi} \cdot \boldsymbol{v}_{\mathfrak{r}} \varrho \xi=\widehat{\psi}\left(\mathcal{R}^{\varrho} \mathbf{v}-\left(\partial_{t} \mathfrak{r}^{\varrho} \xi \boldsymbol{v}\right) \circ \varphi_{\mathfrak{r}^{\varrho} \xi}^{-1}\right) \cdot \boldsymbol{v}_{\mathfrak{r}} \varrho \xi & \text { on } I \times \partial \Omega_{\mathfrak{r}} \varrho \xi(t) \\
\widehat{\psi}(0, \cdot, \cdot)=\widehat{\psi}_{0} \geq 0 & \text { in } \Omega_{\mathfrak{r}} \xi_{0} \times B,
\end{array}
$$

where $\eta_{0}, \eta_{1}: \omega \rightarrow \mathbb{R}$ and $g: I \times \omega \rightarrow \mathbb{R}$ are given functions and

$$
\mathbf{F}(t, \mathbf{y}):=\left(-2 \mu \mathbb{D}_{\mathbf{y}} \mathbf{u}(t, \mathbf{y})-\mathcal{R}^{\varrho} \mathbb{T}(M \widehat{\psi})(t, \mathbf{y})+p(t, \mathbf{y}) \mathbb{I}\right) \boldsymbol{v} \circ \boldsymbol{\varphi}_{\mathfrak{r} \varrho \xi(t)}\left|\operatorname{det} D \boldsymbol{\varphi}_{\mathfrak{r} \varrho \xi(t)}\right|
$$

Let us start with a precise definition of the solution.

Definition 5.3 (Finite energy weak solution) Let (f), $\left.g, \eta_{0}, \widehat{\psi}_{0}, \mathbf{u}_{0}, \eta_{1}, \mathbf{v}, \xi\right)$ be a dataset such that

$$
\begin{aligned}
& \mathbf{f} \in L^{2}\left(\bar{I} ; L_{\mathrm{loc}}^{2}\left(\mathbb{R}^{3} ; \mathbb{R}^{3}\right)\right), \quad g \in L^{2}\left(\bar{I} ; L^{2}(\omega)\right), \quad \eta_{0} \in W^{5,2}(\omega) \text { with }\left\|\eta_{0}\right\|_{L^{\infty}(\omega)}<L, \\
& \widehat{\psi}_{0} \in L^{2}\left(\Omega_{\eta_{0}} ; L_{M}^{2}(B)\right), \quad \mathbf{u}_{0} \in L_{\operatorname{div}_{\mathbf{x}}}^{2}\left(\Omega_{\eta_{0}} ; \mathbb{R}^{3}\right) \text { is such that } \operatorname{tr}_{\eta_{0}} \mathbf{u}_{0}=\eta_{1} \gamma\left(\eta_{0}\right), \quad \eta_{1} \in L^{2}(\omega), \\
& \mathbf{v} \in L_{\operatorname{div}_{\mathbf{x}}}^{2}\left(I \times \mathbb{R}^{3} ; \mathbb{R}^{3}\right), \quad \xi \in L^{p}\left(I ; W^{4, p}(\omega)\right) \text { with }\|\xi\|_{L^{\infty}(I \times \omega)}<L,
\end{aligned}
$$

where $p>2$. In addition, we assume

$$
\Xi_{0} \in L^{\infty}\left(\Omega_{\eta_{0}}\right) \text { where } \Xi_{0}=\int_{B} M(\mathbf{q}) \widehat{\psi}_{0}(\cdot, \mathbf{q}) \text { d } \mathbf{q} \text { in } \Omega_{\eta_{0}} .
$$

We call the triple $(\mathbf{u}, \widehat{\psi}, \eta)$ a finite energy weak solution to the system (5.17)-(5.27) with data $\left(\mathbf{f}, g, \eta_{0}, \widehat{\psi}_{0}, \mathbf{u}_{0}, \eta_{1}, \mathbf{v}, \xi\right)$ provided that the following holds:

(a) the velocity $\mathbf{u}$ satisfies

$$
\mathbf{u} \in L^{\infty}\left(I ; L^{2}\left(\Omega_{\mathfrak{r} \varrho} \varrho(t) ; \mathbb{R}^{3}\right)\right) \cap L^{2}\left(I ; W_{\operatorname{div}_{\mathbf{x}}}^{1,2}\left(\Omega_{\mathfrak{r}} \varrho \xi(t) ; \mathbb{R}^{3}\right)\right) \text { and }
$$

in the sense of traces and $\eta$ satisfies

$$
\eta \in W^{1, \infty}\left(I ; L^{2}(\omega)\right) \cap L^{\infty}\left(I ; W^{5,2}(\omega)\right) \text { with }\|\eta\|_{L^{\infty}(I \times \omega)}<L
$$


and for all $(\phi, \varphi) \in C^{\infty}(\bar{I} \times \omega) \times C^{\infty}\left(\bar{I} \times \mathbb{R}^{3} ; \mathbb{R}^{3}\right)$ with $\phi(T, \cdot)=0, \varphi(T, \cdot)=0$, $\operatorname{div}_{\mathbf{x}} \varphi=0$ and $\operatorname{tr}_{\mathfrak{r}} \varrho \xi \varphi=\phi v$, we have

$$
\begin{aligned}
\int_{I} \frac{\mathrm{d}}{\mathrm{d} t}( & \left.\int_{\Omega_{\mathrm{r}} \varrho_{\xi}(t)} \mathbf{u} \cdot \boldsymbol{\varphi} \mathrm{d} \mathbf{x}+\int_{\omega} \partial_{t} \eta \phi \mathrm{d} \mathbf{y}\right) \mathrm{d} t \\
& =\int_{I} \int_{\Omega_{\mathfrak{r}} \varrho_{\xi(t)}}\left(\mathbf{u} \cdot \partial_{t} \boldsymbol{\varphi}-\frac{1}{2}\left(\mathcal{R}^{\varrho} \mathbf{v} \cdot \nabla\right) \mathbf{u} \cdot \boldsymbol{\varphi}+\frac{1}{2}\left(\mathcal{R}^{\varrho} \mathbf{v} \cdot \nabla\right) \boldsymbol{\varphi} \cdot \mathbf{u}\right) \mathrm{d} \mathbf{x} \mathrm{d} t \\
& -\int_{I} \int_{\Omega_{\mathcal{R} \varrho} \varrho^{(t)}}\left(\mu \nabla_{\mathbf{x}} \mathbf{u}: \nabla_{\mathbf{x}} \boldsymbol{\varphi}+\mathbb{T}(M \widehat{\psi}): \nabla_{\mathbf{x}} \mathcal{R}^{\varrho} \boldsymbol{\varphi}-\mathbf{f} \cdot \boldsymbol{\varphi}\right) \mathrm{d} \mathbf{x} \mathrm{d} t \\
& +\int_{I} \int_{\omega}\left(\partial_{t} \eta \partial_{t} \phi+\frac{1}{2} \partial_{t} \eta \partial_{t} \mathfrak{r}^{\varrho} \eta \phi+g \phi\right) \mathrm{d} \mathbf{y} \mathrm{d} t \\
& -\int_{I}\left(\left\langle K^{\prime}(\xi), \phi\right\rangle+\varrho\left\langle\mathcal{L}^{\prime}(\eta), \phi\right\rangle\right) \mathrm{d} t .
\end{aligned}
$$

(b) the probability density function $\widehat{\psi}$ satisfies:

$$
\begin{aligned}
& \widehat{\psi} \geq 0 \text { a.e. in } I \times \Omega_{\mathfrak{r} \varrho} \varrho \xi(t) \times B, \\
& \widehat{\psi} \in L^{\infty}\left(I ; L^{1}\left(\Omega_{\mathfrak{r} \varrho \xi(t)} ; L_{M}^{1}(B)\right)\right) \text {, } \\
& \mathcal{F}(\widehat{\psi}) \in L^{\infty}\left(I ; L^{1}\left(\Omega_{\mathfrak{r} \varrho} \varrho(t) ; L_{M}^{1}(B)\right)\right), \\
& \sqrt{\widehat{\psi}} \in L^{2}\left(I ; L^{2}\left(\Omega_{\mathfrak{r} \varrho \xi(t)} ; W_{M}^{1,2}(B)\right)\right) \cap L^{2}\left(I ; D^{1,2}\left(\Omega_{\mathfrak{r} \varrho \xi(t)} ; L_{M}^{2}(B)\right)\right), \\
& \Xi(t, \mathbf{x})=\int_{B} M \widehat{\psi}(t, \mathbf{x}, \mathbf{q}) \mathrm{d} \mathbf{q} \in L^{\infty}\left(I \times \Omega_{\mathfrak{r} \varrho \xi(t)}\right) \cap L^{2}\left(I ; W^{1,2}\left(\Omega_{\mathfrak{r} \varrho \xi(t)}\right)\right) ;
\end{aligned}
$$

and for all $\varphi \in C^{\infty}\left(\bar{I} \times \mathbb{R}^{3} \times \bar{B}\right)$, we have

$$
\begin{aligned}
& \int_{I} \frac{\mathrm{d}}{\mathrm{d} t} \int_{\Omega_{\mathrm{r}} \varrho_{\xi(t)} \times B} M \widehat{\psi} \varphi \mathrm{d} \mathbf{q} \mathrm{d} \mathbf{x} \mathrm{d} t=\int_{I \times \Omega_{\mathrm{r}} \varrho_{\xi(t)} \times B}\left(M \widehat{\psi} \partial_{t} \varphi+M \mathcal{R}^{\varrho} \mathbf{v} \widehat{\psi} \cdot \nabla_{\mathbf{x}} \varphi-\varepsilon M \nabla_{\mathbf{x}} \widehat{\psi} \cdot \nabla_{\mathbf{x}} \varphi\right) \mathrm{d} \mathbf{q} \mathrm{d} \mathbf{x} \mathrm{d} t \\
& \quad+\sum_{i=1}^{K} \int_{I \times \Omega_{\mathrm{r}} \xi_{\xi(t)} \times B}\left(M\left(\nabla_{\mathbf{x}} \mathcal{R}^{\varrho} \mathbf{v}\right) \mathbf{q}_{i} \widehat{\psi}-\sum_{j=1}^{K} \frac{A_{i j}}{4 \lambda} M \nabla_{\mathbf{q}_{j}} \widehat{\psi}\right) \cdot \nabla_{\mathbf{q}_{i}} \varphi \mathrm{d} \mathbf{q} \mathrm{d} \mathbf{x} \mathrm{d} t
\end{aligned}
$$

(c) for all $t \in I$, we have

$$
\begin{aligned}
& \int_{\Omega_{\mathfrak{r}} \varrho \xi(t)} \frac{1}{2}|\mathbf{u}(t)|^{2} \mathrm{~d} \mathbf{x}+\int_{\omega} \frac{1}{2}\left|\partial_{t} \eta(t)\right|^{2} \mathrm{~d} \mathbf{y}+\varrho \int_{\omega}\left|\nabla_{\mathbf{y}}^{5} \eta(t)\right|^{2} \mathrm{~d} \mathbf{y}+\mu \int_{0}^{t} \int_{\Omega_{\mathfrak{r}} \varrho \xi(\sigma)}\left|\nabla_{\mathbf{x}} \mathbf{u}\right|^{2} \mathrm{~d} \mathbf{x} \mathrm{d} \sigma \\
& \quad \leq \int_{\Omega_{\mathfrak{r}} \varrho_{\xi_{0}}} \frac{1}{2}\left|\mathbf{u}_{0}\right|^{2} \mathrm{~d} \mathbf{x}+\int_{\omega} \frac{1}{2}\left|\eta_{1}\right|^{2} \mathrm{~d} \mathbf{y}+\varrho \int_{\omega}\left|\nabla_{\mathbf{y}}^{5} \eta_{0}\right|^{2} \mathrm{~d} \mathbf{y}+\int_{0}^{t} \int_{\omega} g \partial_{\sigma} \eta \mathrm{d} \mathbf{y} \mathrm{d} \sigma+\int_{0}^{t}\left\langle K^{\prime}(\xi) \partial_{\sigma} \eta\right\rangle \mathrm{d} \sigma \\
& \quad+\int_{0}^{t} \int_{\Omega_{\mathfrak{r}} \varrho \xi(\sigma)} \mathbf{f} \cdot \mathbf{u} \mathrm{d} \mathbf{x} \mathrm{d} \sigma-\int_{0}^{t} \int_{\Omega_{\mathfrak{r}} \varrho \xi(\sigma)} \mathbb{T}(M \widehat{\psi}): \nabla_{\mathbf{x}} \mathcal{R}^{\varrho} \mathbf{u} \mathrm{d} \mathbf{x} \mathrm{d} \sigma
\end{aligned}
$$


(d) we have the estimate

$$
\begin{aligned}
& \|\Xi(t, \cdot)\|_{L^{\infty}\left(\Omega_{\mathfrak{r}} \varrho_{\xi(t)}\right)}^{2}+k \int_{\Omega_{\mathfrak{r}} \varrho_{\xi(t)} \times B} M \mathcal{F}(\widehat{\psi}(t, \cdot, \cdot)) \mathrm{d} \mathbf{q} \mathrm{d} \mathbf{x}+\varepsilon \int_{0}^{t} \int_{\Omega_{\mathfrak{r}} \varrho_{\xi(\sigma)}}\left|\nabla_{\mathbf{x}} \Xi\right|^{2} \mathrm{~d} \mathbf{x} \mathrm{d} \sigma \\
& +4 k \varepsilon \int_{0}^{t} \int_{\Omega_{\mathfrak{r}} \varrho_{\xi(\sigma)} \times B} M\left|\nabla_{\mathbf{x}} \sqrt{\widehat{\psi}}\right|^{2} \mathrm{~d} \mathbf{q} \mathrm{d} \mathbf{x} \mathrm{d} \sigma+\frac{k A_{0}}{\lambda} \int_{0}^{t} \int_{\Omega_{\mathfrak{r}} \varrho_{\xi(\sigma)} \times B} M\left|\nabla_{\mathbf{q}} \sqrt{\widehat{\psi}}\right|^{2} \mathrm{~d} \mathbf{q} \mathrm{d} \mathbf{x} \mathrm{d} \sigma \\
& \lesssim\left\|\Xi_{0}\right\|_{L^{\infty}\left(\Omega_{\mathfrak{r}} \varrho_{\xi(0)}\right)}^{2}+k \int_{\Omega_{\mathfrak{r}} \varrho_{\xi(0)} \times B} M \mathcal{F}\left(\widehat{\psi}_{0}\right) \mathrm{d} \mathbf{q} \mathrm{d} \mathbf{x} \\
& +\int_{0}^{t} \int_{\Omega_{\mathfrak{r} \varrho \xi(\sigma)}} \mathbb{T}(M \widehat{\psi}): \nabla_{\mathbf{x}} \mathcal{R}^{\varrho} \mathbf{v} \mathrm{d} \mathbf{x} \mathrm{d} \sigma
\end{aligned}
$$

for all $t \in I$.

The following result establishes the existence of a solution, in the sense of Definition 5.3 to the linearized system (5.17)-(5.27), see Remark 2.7 for the case $\lim _{t \rightarrow T}\|\eta(t)\|_{L^{\infty}(\omega)}=L$.

Theorem 5.4 Let (f), $\left.g, \eta_{0}, \widehat{\psi}_{0}, \mathbf{u}_{0}, \eta_{1}, \mathbf{v}, \xi\right)$ be a dataset satisfying (5.28) and (5.29). Then there is a finite energy weak solution (u, $\widehat{\psi}, \eta)$ of the system (5.17)-(5.26) on the interval $I=(0, T)$ in the sense of Definition 5.3. The number $T$ is restricted only if $\lim _{t \rightarrow T}\|\eta(t)\|_{L^{\infty}(\omega)}=L$.

Proof In the linearized problem, the Fokker-Planck equation is decoupled from the fluid-structure problem. Consequently, both can be solved independently. On the one hand, given $\left(\widehat{\psi}_{0}, \eta_{1}, \mathbf{v}, \xi\right)$, let $\widehat{\psi}$ be the solution to the Fokker-Planck equation from Theorem 4.2. On the other hand, given $\widehat{\psi}$, the analysis of the fluid-structure problem (with right-hand side $\mathbf{f}+\mathcal{R}^{\varrho} \operatorname{div}_{\mathbf{x}} \mathbb{T}(M \widehat{\psi})$ in the fluid equation) is very similar to that of Lengeler and Růžička (2014, Prop. 3.27) which is based on a finite-dimensional Galerkin approximation. The distribution $K^{\prime}(\xi)$ can be represented by a function belonging to $L^{2}(I \times \omega)$ as $\xi \in L^{p}\left(I ; W^{4, p}\right)$ (at least if we choose $p$ large enough) and can be put together with the forcing $g$. The higher order operator $\mathcal{L}^{\prime}$ does not change the analysis compared to $\Delta_{\mathbf{x}}^{2}$ (apart from the need for higher order Sobolev spaces). As a consequence, the problem can be treated as in Lengeler and Růžička (2014). In particular, we obtain the energy inequality (5.31) (in fact, the energy inequality in Lengeler and Růžička (2014, Prop. 3.27) slightly differs from (5.31) but a trivial modification leads to the required form).

\subsection{A Fixed Point Argument}

We now seek a fixed point of the solution map $(\mathbf{v}, \xi) \mapsto(\mathbf{u}, \eta)$ on $L^{2}\left(I, L_{\operatorname{div}_{\mathbf{x}}}^{2}\left(\mathbb{R}^{3}\right)\right) \times$ $L^{p}\left(I ; W^{4, p}(\omega)\right)$ from Theorem 5.4. Note that we extend $\mathbf{u}$ by zero to $\mathbb{R}^{3}$. The resulting function is not weekly differentibale anymore but still divergence-free (in the sense of distributions). Since we do not know about uniqueness of the solutions constructed in Theorem 5.4, we will use the following fixed point theorem for set-valued mappings.

Theorem 5.5 (Granas and Dugundji (2003)) Let $C$ be a convex subset of a normed vector space $Z$, let $\mathfrak{P}(C)$ be the power set of $C$ and let $F: C \rightarrow \mathfrak{P}(C)$ be an 
upper-semicontinuous set-valued mapping, that is, for every open set $W \subset C$ the set $\{c \in C: F(c) \in W\} \subset C$ is open. Moreover, let $F(C)$ be contained in a compact subset of $C$, and let $F(c)$ be non-empty, convex and compact for all $c \in C$. Then $F$ possesses a fixed point, that is, there exists some $c_{0} \in C$ with $c_{0} \in F\left(c_{0}\right)$.

We consider the interval $I_{*}=\left(0, T_{*}\right)$ with $T_{*}$ sufficiently small to be chosen later. We consider the set

$$
\mathscr{D}:=\left\{(\xi, \mathbf{v}) \in L^{p}\left(I_{*} ; W^{4, p}(\omega)\right) \cap C\left(\bar{I}_{*} \times \omega\right) \times L^{2}\left(I_{*}, L_{\mathrm{div}_{\mathbf{x}}}^{2}\left(\mathbb{R}^{3}\right)\right): \begin{array}{c}
\|\xi\|_{L^{p}\left(I_{*} ; W^{2, p}(\omega)\right)} \leq M_{1}, \\
\|\xi\|_{L^{\infty}\left(I_{*} \times \omega\right)} \leq M_{1}, \xi(0)=\eta_{0} \\
\|\mathbf{v}\|_{L^{2}\left(I_{*} \times \mathbb{R}^{3}\right)} \leq M_{2}
\end{array}\right\}
$$

for $M_{1}=\left(\left\|\eta_{0}\right\|_{L^{\infty}(\omega)}+L\right) / 2$ and $M_{2}>0$ to be chosen later. Note that the coupling at the boundary between velocity and shell is not contained in the definition of $\mathscr{D}$. This is a feature which one only gains via the fixed point and not before. Let

$$
F: \mathscr{D} \rightarrow \mathfrak{P}(\mathscr{D})
$$

with

$$
F:(\mathbf{v}, \xi) \mapsto\{(\mathbf{u}, \eta):(\mathbf{u}, \eta) \text { solves (5.30) with }(\mathbf{v}, \xi) \text { and satisfies (5.31) }\}
$$

Note that when we say $(\mathbf{u}, \eta)$ solves $(5.30)$ with $(\mathbf{v}, \xi)$ this has to be understood in the sense that there is a solution $\psi$ to the Fokker-Planck equation (with data $(\mathbf{v}, \xi)$ ) which satisfies the corresponding energy inequality (existence of $\psi$ is guaranteed by Theorem 4.2). First of all, we note that the image of $F$ is nonempty due to Theorem 5.4. Next, we have to check that $F(\mathscr{D}) \subset \mathscr{D}$. Given $(\mathbf{v}, \xi)$, we can solve the Fokker-Planck equation with data $\left(\mathcal{R}^{\varrho} \mathbf{v}, \mathfrak{r}^{\varrho} \xi, \widehat{\psi}_{0}\right)$ by means of Theorem 4.2. In particular, we have

$$
\int_{\Omega_{\mathrm{r} \varrho \xi(t)} \times B} M|\widehat{\psi}|^{2} \mathrm{~d} \mathbf{q} \mathrm{d} \mathbf{x} \leq c\left(M_{1}, M_{2}, \varrho\right)
$$

uniformly in $t$. This yields

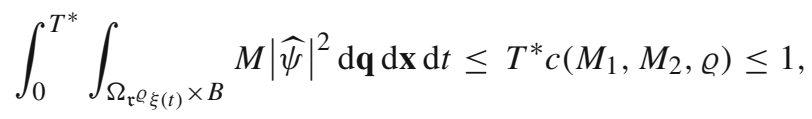

provided we choose $T^{*}$ small enough (depending on $M_{1}$ and $M_{2}$ and $\varrho$ ) and hence

$$
\begin{aligned}
& \int_{0}^{T^{*}} \int_{\Omega_{\mathfrak{r}} \varrho_{\xi(t)} \times B}\left|\mathcal{R}^{\varrho} \operatorname{div}_{\mathbf{x}} \mathbb{T}(M \widehat{\psi})\right|^{2} \mathrm{~d} \mathbf{q} \mathrm{d} \mathbf{x} \mathrm{d} t \leq c(\varrho) \int_{0}^{T^{*}} \int_{\Omega_{\mathfrak{r}} \varrho_{\xi(t)} \times B}|\mathbb{T}(M \widehat{\psi})|^{2} \mathrm{~d} \mathbf{q} \mathrm{d} \mathbf{x} \mathrm{d} t \\
& \leq c(\varrho) \text {. }
\end{aligned}
$$


Now we use the a priori estimate from Theorem 5.4 to conclude

$$
\begin{aligned}
\sup _{I_{*}} \int_{\Omega_{\mathrm{r}} \varrho \xi(t)}|\mathbf{u}|^{2} \mathrm{~d} \mathbf{x} & +\int_{I_{*}} \int_{\Omega_{\mathrm{r}} \varrho \xi(t)}\left|\nabla_{\mathbf{x}} \mathbf{u}\right|^{2} \mathrm{~d} \mathbf{x} \mathrm{d} \sigma+\sup _{I_{*}} \int_{\omega}\left(\left|\partial_{t} \eta\right|^{2}+\left|\nabla_{\mathbf{y}}^{5} \eta\right|^{2}\right) \mathrm{d} \mathbf{y} \\
& \leq c\left(\mathbf{f}, g, K^{\prime}(\xi), \mathbf{u}_{0}, \eta_{0}, \eta_{1}, \varrho\right) \leq c\left(M_{1}\right)
\end{aligned}
$$

independently of $M_{2}$ and the size of $I_{*}$. This implies that $\eta \in C^{\alpha}(\bar{I} \times \omega)$, by Sobolev embedding for some $\alpha>0$, with Hölder norm independent of $M_{1}$ and $M_{2}$. We obtain

$$
|\eta(t, x)| \leq\left|\eta(t, x)-\eta_{0}(x)\right|+\left|\eta_{0}(x)\right| \leq c\left(M_{1}\right)\left(T^{*}\right)^{\alpha}+\left\|\eta_{0}\right\|_{L^{\infty}(\omega)} .
$$

Therefore, we find for $T^{*}$ small enough (but independent of $\mathbf{v}$ and $\xi$ ) such that

$$
\|\eta\|_{L^{\infty}\left(I_{*} \times \omega\right)} \leq M_{1} .
$$

Hence we gain $F(\mathscr{D}) \subset \mathscr{D}$ for an appropriate choice of $M_{2} \in \mathbb{R}_{+}$. Similarly, we obtain

$$
\left(\int_{I_{*}}\|\eta\|_{W^{4, p}(\omega)}^{p} \mathrm{~d} t\right)^{\frac{1}{p}} \leq\left(T^{*}\right)^{\frac{1}{p}}\|\eta\|_{L^{\infty}\left(I_{*} ; W^{4, p}(\omega)\right)} \leq c\left(T^{*}\right)^{\frac{1}{p}}\|\eta\|_{L^{\infty}\left(I_{*} ; W^{5,2}(\omega)\right)} \leq M_{1}
$$

for $T^{*}$ small enough using Sobolev's embedding and (5.34).

Next, since the problem is linear and the left-hand side of the energy inequality is convex, we find that $F(\xi, \mathbf{v})$ is a convex and closed subset of $\mathscr{D}$. Also, we obtain upper-semicontinuity of the set-valued mapping. It remains to show that $F(\mathscr{D})$ is relatively compact. Consider $\left(\eta_{n}, \mathbf{u}_{n}\right)_{n \in \mathbb{N}} \subset F(\mathscr{D})$. Then there exists a corresponding sequence $\left(\xi_{n}, \mathbf{v}_{n}\right)_{n \in \mathbb{N}} \subset \mathscr{D}$, such that $\left(\eta_{n}, \mathbf{u}_{n}\right)$ solves $(5.30)$, with respect to $\left(\mathbf{v}_{n}, \xi_{n}\right)$. The corresponding solution to the Fokker-Planck equation will be denoted by $\widehat{\psi}_{n}$. Due to the estimates above, we may choose subsequences such that

$$
\begin{gathered}
\eta_{n} \vec{*}^{*} \eta \quad \text { in } L^{\infty}\left(I_{*}, W^{5,2}(\omega)\right), \\
\left.\partial_{t} \eta_{n} \vec{*}^{*} \partial_{t} \eta \quad \text { in } L^{\infty}\left(I_{*}, L^{2}(\omega)\right)\right), \\
\eta_{n} \rightarrow \eta \text { in } L^{2}\left(I_{*}, W^{2,2}(\omega)\right), \\
\mathbf{u}_{n} \rightarrow^{*, \eta} \mathbf{u} \text { in } L^{\infty}\left(I_{*} ; L^{2}\left(\Omega_{\mathfrak{r} \varrho \xi(t)} ; \mathbb{R}^{3}\right)\right), \\
\nabla_{\mathbf{x}} \mathbf{u}_{n} \rightarrow{ }^{\eta} \nabla_{\mathbf{x}} \mathbf{u} \quad \text { in } L^{2}\left(I_{*} ; L^{2}\left(\Omega_{\mathfrak{r} \varrho \xi(t)} ; \mathbb{R}^{3 \times 3}\right)\right), \\
\mathcal{R}^{\varrho} \operatorname{div}_{\mathbf{x}} \mathbb{T}\left(M \widehat{\psi}_{n}\right) \rightarrow \mathcal{R}^{\varrho} \operatorname{div}_{\mathbf{x}} \mathbb{T}(M \widehat{\psi}) \text { in } L^{2}\left(I_{*} \times \Omega_{\mathfrak{r} \varrho \xi(t)} ; \mathbb{R}^{3}\right),
\end{gathered}
$$

where we also used linearity of $\mathbb{T}$. The compactness of $\eta_{n}$ in $C\left(\bar{I}_{*} \times \omega\right)$ follows immediately by Arcela-Ascoli's theorem, since we know that $\eta_{n}$ is uniformly Hölder continuous. Compactness in $L^{p}\left(I_{*} ; W^{4, p}(\omega)\right)$ then follows from Aubin-Lions' Lemma (recall (5.36) and (5.37)) and interpolation. The proof of the compactness of $\mathbf{u}_{n}$ is much more sophisticated. Fortunately, we can follow Lengeler and Růžička (2014, Proposition 3.34) which is based on the compactness arguments Lengeler and Růžička 
(2014, Section 3.1). The only difference is that we have a sequence of forcing terms $\mathbf{f}+\mathcal{R}^{\varrho} \operatorname{div}_{\mathbf{x}} \mathbb{T}\left(M \widehat{\psi}_{n}\right)$. But the term coming from the extra stress is bounded in $L^{2}$ due to (5.33) so the argument remains unchanged and we conclude. Note also that we can extend $\mathbf{u}_{n}$ and $\mathbf{u}$ by zero to the whole space and gain

$$
\mathbf{u}_{n} \rightarrow \mathbf{u} \text { in } L^{2}\left(I_{*} ; L_{\operatorname{div}_{\mathbf{x}}}^{2}\left(\mathbb{R}^{3} ; \mathbb{R}^{3}\right)\right)
$$

Finally, we find for all $k, l \in \mathbb{N}$ that $\left\|\partial_{t}^{l} \nabla^{k} \mathfrak{r}^{\varrho} \xi_{n}\right\|_{L^{\infty}\left(I_{*} \times \omega\right)} \leq c(\kappa, k, l)$. Hence, there is a subsequence (not relabelled) such that

$$
\mathfrak{r}^{\varrho} \xi_{n} \rightarrow \mathfrak{r}^{\varrho} \xi \text { in } C^{2}\left(\bar{I}_{*} \times \omega\right)
$$

The proof of the compactness is complete and the existence of a fixed point follows by Theorem 5.5 .

Finally, we add (5.31) and (5.32) (with $(\xi, \mathbf{v})=(\eta, \mathbf{u}))$ to obtain the energy inequality (5.16) which completes the proof of Theorem 5.2. Note that with $\xi=\eta$ we can rewrite

$$
\int_{0}^{t}\left\langle K^{\prime}(\xi), \partial_{\sigma} \eta\right\rangle \mathrm{d} \sigma=\int_{0}^{t}\left\langle K^{\prime}(\eta), \partial_{\sigma} \eta\right\rangle \mathrm{d} \sigma=\int_{0}^{t} \frac{\mathrm{d}}{\mathrm{d} \sigma} K(\eta) \mathrm{d} \sigma=K(\eta(t))-K\left(\eta_{0}\right)
$$

\section{The Limit $Q \rightarrow 0$}

We consider a sequence of smooth functions $\left(\eta_{0}^{\varrho}, \eta_{1}^{\varrho}, \mathbf{u}_{0}^{\varrho}\right)$ such that

$$
\begin{aligned}
& \eta_{0}^{\varrho} \rightarrow \eta_{0} \quad \text { in } \quad W^{2,2}(\omega), \quad \eta_{1}^{\varrho} \rightarrow \eta_{1} \quad \text { in } \quad L^{2}(\omega), \sqrt{\varrho} \eta_{0}^{\varrho} \rightarrow 0 \text { in } W^{5,2}(\omega), \\
& \mathbf{u}_{0}^{\varrho} \rightarrow \mathbf{u}_{0} \text { in } L^{2}\left(\mathbb{R}^{3}\right),
\end{aligned}
$$

as $\varrho \rightarrow 0$. Here $\mathbf{u}_{0}$ has been extended by zero to the whole space and $\mathbf{u}_{0}^{\varrho}$ is smooth and divergence-free approximation which satisfies $\operatorname{tr}_{\mathfrak{r}} \eta_{\eta} \mathbf{u}_{0}^{\varrho}=\eta_{1}^{\varrho} \gamma\left(\eta_{0}^{\varrho}\right)$, cf. Lengeler and Růžička (2014, Sec. 3.2). For a fixed $\varrho>0$, we apply Theorem 5.2 to obtain a sequence of solutions $\left(\mathbf{u}^{\varrho}, \widehat{\psi}^{\varrho}, \eta^{\varrho}\right)$ to the regularized problem (5.2)-(5.11) with data $\left(\mathbf{f}, g, \eta_{0}^{\varrho}, \widehat{\psi}_{0}, \mathbf{u}_{0}, \eta_{1}^{\varrho}\right)$. The forthcoming effort is to pass with $\varrho \rightarrow 0$ which will prove Theorem 2.6. We split this prove into two subsections. In the first part, we establish uniform a priori estimates. In particular, we prove fractional differentiability of the shell which eventually allows to pass to the limit in the nonlinear term $K\left(\eta^{\varrho}\right)$. Finally, we have to pass to the limit in the nonlinear terms in the momentum equations as well as the Fokker-Planck equations. In the case of fixed domains, this is done by classical compactness tools like the classical Aubin-Lions lemma. While one can still use the equations to gain information on the time-regularity of $\mathbf{u}^{\varrho}$ and $\widehat{\psi}^{\varrho}$, the analysis is significantly more involved. 


\subsection{A Priori Estimate}

Using (5.16) and applying Young's inequality, we obtain

$$
\begin{aligned}
& \int_{\Omega_{\mathfrak{r}{ }^{\varrho} \eta^{\varrho}(t)}}\left|\mathbf{u}^{\varrho}(t)\right|^{2} \mathrm{~d} \mathbf{x}+\mu \int_{0}^{t} \int_{\Omega_{\mathrm{r} \varrho \eta \eta^{\varrho}(\sigma)}}\left|\nabla_{\mathbf{x}} \mathbf{u}^{\varrho}\right|^{2} \mathrm{~d} \mathbf{x} \mathrm{d} \sigma+\int_{\omega}\left|\partial_{t} \eta^{\varrho}(t)\right|^{2} \mathrm{~d} \mathbf{y}+\varrho \int_{\omega}\left|\nabla_{\mathbf{y}}^{5} \eta^{\varrho}(t)\right|^{2} \mathrm{~d} \mathbf{y} \\
& +K\left(\eta^{\varrho}(t)\right)+\left\|\Xi^{\varrho}(t, \cdot)\right\|_{L^{\infty}\left(\Omega_{\mathfrak{r}} \varrho_{\eta{ }^{(}(t)}\right)}^{2}+\varepsilon \int_{0}^{t} \int_{\Omega_{\mathfrak{r}} \varrho_{\eta} \eta_{(\sigma)}}\left|\nabla_{\mathbf{x}} \Xi^{\varrho}\right|^{2} \mathrm{~d} \mathbf{x} \mathrm{d} \sigma
\end{aligned}
$$

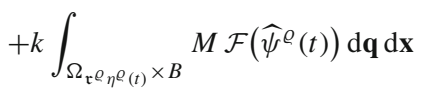

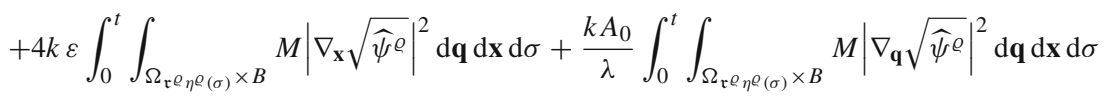

$$
\begin{aligned}
& \lesssim \int_{\Omega_{\mathfrak{r} \varrho} \eta_{0}^{\varrho}}\left|\mathbf{u}_{0}\right|^{2} \mathrm{~d} \mathbf{x}+\int_{\omega}\left|\eta_{1}^{\varrho}\right|^{2} \mathrm{~d} \mathbf{y}+K\left(\eta_{0}^{\varrho}\right)+\varrho \int_{\omega}\left|\nabla_{\mathbf{y}}^{5} \eta_{0}^{\varrho}\right|^{2} \mathrm{~d} \mathbf{y}+\left\|\Xi_{0}^{\varrho}\right\|_{L^{\infty}\left(\Omega_{\mathrm{r}} \eta_{0} \rho\right)}^{2} \\
& +k \int_{\Omega_{\mathrm{r} \varrho} \eta_{0}^{\varrho} \times B} M \mathcal{F}\left(\widehat{\psi}_{0}\right)+\int_{0}^{t} \int_{\Omega_{\mathrm{r} \varrho} \eta^{\varrho}(\sigma)}|\mathbf{f}|^{2} \mathrm{~d} \mathbf{x} \mathrm{d} \sigma \int_{0}^{t} \int_{\omega}|g|^{2} \mathrm{~d} \mathbf{y} \mathrm{d} \sigma
\end{aligned}
$$

for all $t \in I$ where the right-hand side is uniformly bounded due to (6.1) and (5.13). Passing to a non-relabelled subsequence, we conclude

$$
\begin{aligned}
& \eta^{\varrho} \rightarrow^{*} \eta \quad \text { in } L^{\infty}\left(I, W^{2,2}(\omega)\right), \\
& \left.\partial_{t} \eta^{\varrho} \longrightarrow^{*} \partial_{t} \eta \quad \text { in } L^{\infty}\left(I, L^{2}(\omega)\right)\right) \text {, } \\
& \varrho \eta^{\varrho} \rightarrow 0 \text { in } L^{\infty}\left(I, W^{5,2}(\omega)\right) \text {, } \\
& \mathbf{u}^{\varrho} \rightarrow^{*, \eta} \mathbf{u} \text { in } L^{\infty}\left(I ; L^{2}\left(\Omega_{\mathfrak{r}} \eta^{\varrho}(t) ; \mathbb{R}^{3}\right)\right), \\
& \nabla_{\mathbf{x}} \mathbf{u}^{\varrho} \rightarrow{ }^{\eta} \nabla_{\mathbf{x}} \mathbf{u} \text { in } L^{2}\left(I ; L^{2}\left(\Omega_{\mathfrak{r}} \eta^{\varrho}(t) ; \mathbb{R}^{3 \times 3}\right)\right), \\
& \Xi^{\varrho} \rightarrow^{*, \eta} \Xi \text { in } L^{\infty}\left(I \times \Omega_{\mathcal{R}^{\%} \eta(t)}\right), \\
& \Xi^{\varrho} \rightarrow{ }^{\eta} \Xi \text { in } L^{2}\left(I ; W^{1,2}\left(\Omega_{\mathfrak{r} \varrho} \eta^{\varrho}(t)\right)\right), \\
& \widehat{\psi}^{\varrho} \rightarrow^{*, \eta} \widehat{\psi} \quad \text { in } L^{\infty}\left(I ; L^{1}\left(\Omega_{\mathfrak{r} \varrho} \eta^{\varrho}(t) ; L_{M}^{1}(B)\right)\right) \text {, } \\
& \sqrt{\widehat{\psi}^{\varrho}} \rightarrow \eta \sqrt{\widehat{\psi}} \text { in } L^{2}\left(I ; L^{2}\left(\Omega_{\mathfrak{r} \varrho} \eta^{\varrho}(t) ; W_{M}^{1,2}(B)\right)\right) \text {, } \\
& \sqrt{\widehat{\psi} \varrho} \rightarrow \eta \sqrt{\widehat{\psi}} \text { in } L^{2}\left(I ; D^{1,2}\left(\Omega_{\mathfrak{r} \varrho} \eta^{\varrho}(t) ; L_{M}^{2}(B)\right)\right) \text {, }
\end{aligned}
$$

for some limit functions $(\mathbf{u}, \widehat{\psi}, \eta)$ and $\Xi=\int_{B} M \widehat{\psi}$ dq. From (6.8)-(6.12) we obtain in particular

$$
\mathbb{T}\left(M \widehat{\psi}^{\varrho}\right) \rightarrow \mathbb{T}(M \widehat{\psi}) \quad \text { in } \quad L^{2}\left(I \times \Omega_{\mathfrak{r} \varrho} \eta^{\varrho}(t) ; \mathbb{R}^{3 \times 3}\right)
$$

In the following we are going to prove that

$$
\int_{I}\left\|\eta^{\varrho}\right\|_{W^{2+s, 2}(\omega)}^{2} \mathrm{~d} t
$$


is uniformly bounded for some $s>0$. This will be achieved by using an appropriate test-function in the shell equation. As the shell equation is coupled with the fluid equation, we need a suitable test-function for the fluid equation as well. As shown in (Muha and Schwarzacher 2019, Sec. 3), for a given $\eta \in L^{\infty}\left(I ; W^{1,2}(\omega)\right)$ with $\|\eta\|_{L^{\infty}(I \times \omega)}<\ell<L$, there are linear operators

$\mathscr{K}_{\eta}: L^{1}(\omega) \rightarrow \mathbb{R}, \quad \mathscr{F}_{\eta}:\left\{\xi \in L^{1}\left(I ; W^{1,1}(\omega)\right): \mathscr{K}_{\eta}(\xi)=0\right\} \rightarrow L^{1}\left(I ; W_{\operatorname{div}_{\mathbf{x}}}^{1,1}\left(S_{\ell} \cup \Omega\right)\right)$,

such that the tuple $\left(\mathscr{F}_{\eta}\left(\xi-\mathscr{K}_{\eta}(\xi)\right), \xi-\mathscr{K}_{\eta}(\xi)\right)$ satisfies

$$
\begin{gathered}
\mathscr{F}_{\eta}\left(\xi-\mathscr{K}_{\eta}(\xi)\right) \in L^{\infty}\left(I ; L^{2}\left(\Omega_{\eta}\right)\right) \cap L^{2}\left(I ; W_{\operatorname{div}_{\mathbf{x}}}^{1,2}\left(\Omega_{\eta}\right)\right), \\
\xi-\mathscr{K}_{\eta}(\xi) \in L^{\infty}\left(I ; W^{2,2}(\omega)\right) \cap W^{1, \infty}\left(I ; L^{2}(\omega)\right), \\
\operatorname{tr}_{\eta}\left(\mathscr{F}_{\eta}\left(\xi-\mathscr{K}_{\eta}(\xi)\right)=\xi-\mathscr{K}_{\eta}(\xi),\right.
\end{gathered}
$$

provided we have $\eta \in L^{\infty}\left(I ; W^{2,2}(\omega)\right) \cap W^{1, \infty}\left(I ; L^{2}(\omega)\right)$. In particular, we have the estimates

$$
\begin{aligned}
\left\|\mathscr{F}_{\eta}\left(\xi-\mathscr{K}_{\eta}(\xi)\right)\right\|_{L^{q}\left(I ; W^{1, p}\left(S_{\ell} \cup \Omega\right)\right)} & \lesssim\|\xi\|_{L^{q}\left(I ; W^{1, p}(\omega)\right)} \\
& +\left\|\xi \nabla_{\mathbf{x}} \eta\right\|_{L^{q}\left(I ; L^{p}(\omega)\right)} \\
\left\|\partial_{t}\left(\mathscr{F}_{\eta}\left(\xi-\mathscr{K}_{\eta}(\xi)\right)\right)\right\|_{L^{q}\left(I ; L^{p}\left(S_{\ell} \cup \Omega\right)\right)} & \lesssim\left\|\partial_{t} \xi\right\|_{L^{q}\left(I ; L^{p}(\omega)\right)} \\
& +\left\|\xi \partial_{t} \eta\right\|_{L^{q}\left(I ; L^{p}(\omega)\right)}
\end{aligned}
$$

for any $q \in[1, \infty]$ and $p \in(1, \infty)$ as proved in (Muha and Schwarzacher 2019, Prop. 3.3). We now use the test-function

$$
\left(\varphi^{\varrho}, \phi^{\varrho}\right)=\left(\mathscr{F}_{\eta^{\varrho}}\left(\Delta_{-h}^{s} \Delta_{h}^{s} \eta^{\varrho}-\mathscr{K}_{\eta^{\varrho}}\left(\Delta_{-h}^{s} \Delta_{h}^{s} \eta^{\varrho}\right)\right), \Delta_{-h}^{s} \Delta_{h}^{s} \eta^{\varrho}-\mathscr{K}_{\eta^{\varrho}}\left(\Delta_{-h}^{s} \Delta_{h}^{s} \eta^{\varrho}\right)\right)
$$

in equation (5.15). Here $\Delta_{s}^{h} v(\mathbf{y})=h^{-s}\left(v\left(\mathbf{y}+h \boldsymbol{e}_{\alpha}\right)-v(\mathbf{y})\right)$ is the fractional difference quotient in direction $\boldsymbol{e}_{\alpha}$ for $\alpha \in\{1,2\}$. We obtain

$$
\begin{aligned}
& \int_{I}\left(\left\langle K^{\prime}\left(\eta^{\varrho}\right), \phi^{\varrho}\right\rangle+\varrho\left\langle\mathcal{L}^{\prime}\left(\eta^{\varrho}\right), \phi^{\varrho}\right\rangle\right) \mathrm{d} \mathbf{y} \mathrm{d} t \\
& =\int_{I} \int_{\Omega_{\mathfrak{r}{ } \eta{ }^{(t)}}}\left(\mathbf{u}^{\varrho} \cdot \partial_{t} \varphi^{\varrho}+\frac{1}{2}\left(\mathcal{R}^{\varrho} \mathbf{u}^{\varrho} \cdot \nabla\right) \mathbf{u}^{\varrho} \cdot \boldsymbol{\varphi}^{\varrho}\right. \\
& \left.-\frac{1}{2}\left(\mathcal{R}^{\varrho} \mathbf{u}^{\varrho} \cdot \nabla_{\mathbf{x}}\right) \varphi^{\varrho} \cdot \mathbf{u}^{\varrho}-\mu \nabla_{\mathbf{x}} \mathbf{u}^{\varrho}: \nabla_{\mathbf{x}} \varphi^{\varrho}+\mathbf{f} \cdot \boldsymbol{\varphi}^{\varrho}\right) \mathrm{d} \mathbf{x} \mathrm{d} t \\
& -\int_{I} \frac{\mathrm{d}}{\mathrm{d} t}\left(\int_{\Omega_{\mathfrak{r}} \varrho_{\eta} \eta_{(t)}} \mathbf{u}^{\varrho} \cdot \varphi^{\varrho} \mathrm{d} \mathbf{x}+\int_{\omega} \partial_{t} \eta^{\varrho} \phi^{\varrho} \mathrm{d} \mathbf{y}\right) \mathrm{d} t+\int_{I} \int_{\omega}\left(\partial_{t} \eta^{\varrho} \partial_{t} \phi^{\varrho}+g \phi^{\varrho}\right) \mathrm{d} \mathbf{y} \mathrm{d} t \\
& +\int_{I} \int_{\omega} \frac{1}{2} \partial_{t} \eta^{\varrho} \partial_{t} \mathfrak{r}^{\varrho} \eta^{\varrho} \phi^{\varrho} \mathrm{d} \mathbf{x} \mathrm{d} t-\int_{I} \int_{\Omega_{\mathfrak{r}} \eta^{\varrho}(t)} \mathbb{T}\left(M \widehat{\psi}^{\varrho}\right): \nabla_{\mathbf{x}} \mathcal{R}^{\varrho} \boldsymbol{\varphi}^{\varrho} \mathrm{d} \mathbf{x} \mathrm{d} t \\
& =:(I)^{\varrho}+(I I)^{\varrho}+(I I I)^{\varrho}+(I V)^{\varrho}+(V)^{\varrho} \text {. }
\end{aligned}
$$


Since $\eta^{\varrho} \in L^{\infty}\left(I, W^{2,2}(\omega)\right)$ uniformly, due to (Muha and Schwarzacher 2019, Lemma 4.5), we have

$$
\begin{aligned}
\int_{I}\left\|\Delta_{h}^{s} \nabla^{2} \eta^{\varrho}\right\|_{L^{2}(\omega)}^{2} \mathrm{~d} t & \lesssim 1+\int_{I}\left\langle K^{\prime}\left(\eta^{\varrho}\right), \phi^{\varrho}\right\rangle \mathrm{d} t \\
& \lesssim 1+\int_{I}\left(\left\langle K^{\prime}\left(\eta^{\varrho}\right) \phi^{\varrho}\right\rangle+\varrho\left\langle\mathcal{L}^{\prime}\left(\eta^{\varrho}\right), \phi^{\varrho}\right\rangle\right) \mathrm{d} t
\end{aligned}
$$

for every $h>0$ and $s \in\left(0, \frac{1}{2}\right)$ so that our task consists of establishing uniform estimates for the terms $(I)^{\varrho}, \ldots,(V)^{\varrho}$. As in (Muha and Schwarzacher 2019, Sec. 4.2), we obtain the required bounds for $(I)^{\varrho},(I I)^{\varrho}$ and $(I I I)^{\varrho}$. A key ingredient in these estimates is the observation that

$$
\left.\partial_{t} \eta^{\varrho} \in L^{2}\left(I, W^{s, 2}(\omega)\right)\right)
$$

for all $s \in\left(0, \frac{1}{2}\right)$ by the use of (6.7) and the trace theorem, cf. Lemma 2.3. In fact, we can transfer the regularity from $\mathbf{u}^{\varrho}$ to $\partial_{t} \eta^{\varrho}$ by means of the boundary condition $\operatorname{tr}_{\mathcal{R}} \eta^{\varrho} \mathbf{u}^{\varrho}=\partial_{t} \eta^{\varrho} \gamma\left(\eta^{\varrho}\right)$. Using (6.3) and (6.4), we obtain

$$
\begin{aligned}
(I V)^{\varrho} & \leq\left\|\partial_{t} \eta^{\varrho}\right\|_{L^{\infty}\left(I ; L^{2}(\omega)\right)}\left\|\partial_{t} \mathfrak{r}^{\varrho} \eta^{\varrho}\right\|_{L^{\infty}\left(I ; L^{2}(\omega)\right)}\left\|\phi^{\varrho}\right\|_{L^{\infty}\left(I ; L^{\infty}(\omega)\right)} \\
& \lesssim\left\|\partial_{t} \eta^{\varrho}\right\|_{L^{\infty}\left(I ; L^{2}(\omega)\right)}^{2}\left\|\Delta_{-h}^{s} \Delta_{h}^{s} \eta^{\varrho}\right\|_{L^{\infty}\left(I ; W^{1, p}(\omega)\right)} \\
& \lesssim\left\|\eta^{\varrho}\right\|_{L^{\infty}\left(I ; W^{1+2 s, p}(\omega)\right)} \lesssim\left\|\eta^{\varrho}\right\|_{L^{\infty}\left(I ; W^{2,2}(\omega)\right)} \leq c
\end{aligned}
$$

for any $s<\frac{1}{2}$. Here, we have chosen $p \in\left(2, \frac{1}{s}\right]$ such that the Sobolev embeddings $W^{1, p}(\omega) \hookrightarrow L^{\infty}(\omega)$ and $W^{2,2}(\omega) \hookrightarrow W^{1+2 s, p}(\omega)$ holds. We are left with the term $(V)^{\varrho}$ for the mesoscopic coupling. We obtain

$$
\begin{aligned}
(V)^{\varrho} & \left.\leq\left\|\mathbb{T}\left(M \widehat{\psi}^{\varrho}\right)\right\|_{L^{2}\left(I \times \Omega_{\mathrm{r}} \varrho_{\eta}{ }^{(t)}\right)}\left\|\nabla_{\mathbf{x}} \mathcal{R}^{\varrho} \boldsymbol{\varphi}^{\varrho}\right\|_{L^{2}\left(I \times \Omega_{\mathrm{r}} \eta_{\eta}{ }^{\varrho}(t)\right.}\right) \\
& \lesssim\left\|\Delta_{-h}^{s} \Delta_{h}^{s} \eta^{\varrho}\right\|_{L^{2}\left(I ; W^{1,2}(\omega)\right)}+\left\|\left(\Delta_{-h}^{s} \Delta_{h}^{s} \eta^{\varrho}\right) \nabla_{\mathbf{x}} \eta^{\varrho}\right\|_{L^{2}\left(I ; L^{2}(\omega)\right)} \\
& \lesssim\left\|\eta^{\varrho}\right\|_{L^{\infty}\left(I ; W^{1+2 s, 2}(\omega)\right)}+\left\|\eta^{\varrho}\right\|_{L^{\infty}\left(I ; W^{2 s, 4}(\omega)\right.}\left\|\eta^{\varrho}\right\|_{L^{\infty}\left(I ; W^{1,4}(\omega)\right)}
\end{aligned}
$$

using (6.13) and (6.15). Using (6.3), the right-hand side is clearly bounded for all $s<\frac{1}{2}$. Combining all the previous estimates and passing with $h \rightarrow 0$ yields (6.14).

\subsection{Compactness}

To show compactness of the velocity, we are going to apply the method from Lengeler and Růžička (2014). We aim to prove

$$
\begin{aligned}
\int_{I} \int_{\Omega_{\mathfrak{r}} \varrho_{\eta} \varrho_{(t)}}\left|\mathbf{u}^{\varrho}\right|^{2} \mathrm{~d} \mathbf{x} \mathrm{d} t+\int_{I} \int_{\omega}\left|\partial_{t} \eta^{\varrho}\right|^{2} \mathrm{~d} \mathbf{y} \mathrm{d} t \\
\longrightarrow \int_{I} \int_{\Omega_{\eta(t)}}|\mathbf{u}|^{2} \mathrm{~d} \mathbf{x} \mathrm{d} t+\int_{I} \int_{\omega}\left|\partial_{t} \eta\right|^{2} \mathrm{~d} \mathbf{y} \mathrm{d} t
\end{aligned}
$$


which will be a consequence of

$$
\begin{aligned}
\int_{I} \int_{\Omega_{\mathfrak{r}} \eta_{\eta} \varrho(t)} \mathbf{u}_{n} \cdot \mathscr{F}_{\mathfrak{r}} \eta^{\varrho}\left(\partial_{t} \eta^{\varrho}\right) \mathrm{d} \mathbf{x} \mathrm{d} t+\int_{I} \int_{\omega}\left|\partial_{t} \eta^{\varrho}\right|^{2} \mathrm{~d} \mathbf{y} \mathrm{d} t \\
\\
\longrightarrow \int_{I} \int_{\Omega_{\eta(t)}} \mathbf{u} \cdot \mathscr{F}_{\eta}\left(\partial_{t} \eta\right) \mathrm{d} \mathbf{x} \mathrm{d} t+\int_{I} \int_{\omega}\left|\partial_{t} \eta\right|^{2} \mathrm{~d} \mathbf{y} \mathrm{d} t
\end{aligned}
$$

and

$$
\begin{aligned}
& \int_{I} \int_{\Omega_{\mathfrak{r}} \eta_{\eta} \eta^{(t)}} \mathbf{u}^{\varrho} \cdot\left(\mathbf{u}^{\varrho}-\mathscr{F}_{\mathfrak{r}} \eta^{\varrho}{ }\left(\partial_{t} \eta^{\varrho}\right)\right) \mathrm{d} \mathbf{x} \mathrm{d} t \\
& \longrightarrow \int_{I} \int_{\Omega_{\eta(t)}} \mathbf{u} \cdot\left(\mathbf{u}-\mathscr{F}_{\eta}\left(\partial_{t} \eta_{n}\right)\right) \mathrm{d} \mathbf{x} \mathrm{d} t .
\end{aligned}
$$

In order to prove (6.20), we take the test-function $\left(b, \mathscr{F}_{\mathcal{R}} \eta^{\varrho}{ }^{\varrho}\left(b-\mathscr{K}_{\mathfrak{r}}{ } \eta^{\varrho}(b)\right)\right)$ with $b \in W^{5,2}(\omega)$ and $\|b\|_{W^{5,2}(\omega)} \leq 1$. It satisfies for all $q<\infty$,

$$
\begin{aligned}
& \| \partial_{t}\left(\mathscr{F}_{\mathfrak{r}} \eta_{\eta} \varrho\left(b-\mathscr{K}_{\mathfrak{r} \varrho} \eta^{\varrho}(b)\right) \|_{L^{\infty}\left(I ; L^{2}\left(\Omega_{\mathfrak{r}} \varrho_{\eta} \varrho_{(t)}\right)\right.} \leq c,\right. \\
& \left\|\mathscr{F}_{\mathfrak{r} \varrho} \eta\left(b-\mathscr{K}_{\mathfrak{r} \varrho}(b)\right)\right\|_{L^{\infty}\left(I ; W^{1, q}\left(\Omega_{\mathfrak{r}} \varrho_{\eta} \varrho_{(t)}\right)\right)} \leq c,
\end{aligned}
$$

due to (6.15) and (6.16) in combination with (6.3) and (6.4). We consider the functions

$$
\begin{aligned}
& c_{b, \varrho}(t)=\int_{\Omega_{\mathfrak{r}} \eta_{\eta} \varrho(t)} \mathbf{u}^{\varrho} \cdot \mathscr{F}_{\mathfrak{r}} \eta^{\varrho}\left(b-\mathscr{K}_{\mathfrak{r}} \varrho \eta^{\varrho}\right. \\
& c_{b}(t)=\int_{\Omega_{\eta(t)}} \mathbf{u} \cdot \mathscr{F}_{\eta}\left(b-\mathscr{K}_{\eta}(b)\right) \mathrm{d} \mathbf{x} \mathbf{x}+\int_{\omega} \partial_{t} \eta^{\varrho} b \mathrm{~d} \mathbf{y}, \\
& \partial_{t} \eta b \mathrm{~d} \mathbf{y},
\end{aligned}
$$

and aim to show

$$
\left\|c_{b, \varrho}\right\|_{C^{0,1 / \chi^{\prime}(\bar{I})}} \lesssim 1
$$

for some $\chi>1$ uniformly in $b$ and $\varrho$. This follows by inserting $\mathbb{I}_{(0, t)} \mathscr{F}_{\mathfrak{r}} \eta \varrho(b-$ $\left.\mathscr{K}_{\mathfrak{r}} \eta^{\varrho}(b)\right)$ as a test-function in (5.15) provided all terms in in (5.15) have integrability $\chi$. This is indeed the case as a consequence of (6.3)-(6.13). Applying Arcela-Ascoli's theorem we can assume that $c_{b, \varrho}$ converges (for a fixed $b$ ) uniformly in $\bar{I}$, at least for a subsequence. Together with (6.23), this implies uniform convergence of the function

$$
t \mapsto \sup _{\|b\|_{W^{5,2}(\omega)} \leq 1}\left(c_{b, \varrho_{n}}(t)-c_{\varphi}(t)\right)
$$


for a subsequence $\left(\varrho_{n}\right)_{n \in \mathbb{N}}$, cf. Lengeler and Růžička (2014, pages 229, 230) for more details. In particular, we obtain

$$
\left.\int_{I\|b\|_{W^{5,2}(\omega)} \leq 1} \sup _{b, \varrho_{n}}(t)-c_{b}(t)\right) \mathrm{d} t \rightarrow 0
$$

as $n \rightarrow \infty$. By interpolation, we have

$$
\begin{aligned}
& \int_{I\|b\|_{L^{2}(\omega)} \leq 1}\left(c_{b, \varrho_{n}}(t)-c_{b}(t)\right) \mathrm{d} t \leq \varepsilon \int_{I}\left(\left\|\mathbf{u}^{\varrho_{n}}\right\|_{W^{1,2}\left(\mathfrak{r} \varrho n \eta \eta^{\varrho n}\right)}^{2}+\|\mathbf{u}\|_{W^{1,2}\left(\mathfrak{r} \varrho^{\ell} \eta^{\varrho n}\right)}\right) \\
& +\varepsilon \int_{I}\left(\left\|\partial_{t} \eta^{\varrho_{n}}\right\|_{W^{s, 2}(\omega)}+\left\|\partial_{t} \eta\right\|_{W^{s, 2}(\omega)}\right) \\
& \left.+c_{\varepsilon} \int_{I\|b\|_{W^{5,2}(\omega)} \leq 1} \sup _{b, \varrho_{n}}(t)-c_{b}(t)\right) \mathrm{d} t
\end{aligned}
$$

for all $\varepsilon>0$ and all $s \in(0,1 / 2)$. Consequently, we obtain

$$
\int_{I\|b\|_{L^{2}(\omega)} \leq 1}\left(c_{b, \varrho_{n}}(t)-c_{b}(t)\right) \mathrm{d} t \rightarrow 0
$$

as $n \rightarrow \infty$ on account of (6.7), (6.14) and (6.24). Finally, by (6.4) and (6.25) we have

$$
\int_{I}\left(c_{\partial_{t} \eta^{\varrho n}, \varrho_{n}}(t)-c_{\partial_{t} \eta^{\varrho n}}(t)\right) \mathrm{d} t \rightarrow 0
$$

as $n \rightarrow \infty$ which implies (6.20). For the convergence in (6.21), one uses the fact that the function $\mathbf{u}^{\varrho_{n}}-\mathscr{F}_{\mathfrak{r}} \varrho_{n} \eta_{\ell_{n}}\left(\partial_{t} \eta^{\varrho_{n}}\right)$ is zero on $\partial \Omega_{\mathfrak{r} \varrho_{n}} \eta_{n}$ by construction. Hence (6.21) is not affected by the shell equation

and can be proved as in Lengeler and Růžička (2014, pages 213,232). We conclude that (6.19) holds and obtain

$$
\begin{aligned}
& \eta^{\varrho_{n}} \rightarrow \eta \quad \text { in } \quad L^{2}\left(I, W^{2,2}(\omega)\right), \\
& \mathbf{u}^{\varrho_{n}} \rightarrow{ }^{\eta} \mathbf{u} \quad \text { in } \quad L^{2}\left(I ; L^{2}\left(\Omega_{\mathfrak{r} \varrho_{n}} \eta^{\varrho_{n}(t)} ; \mathbb{R}^{3}\right)\right),
\end{aligned}
$$

by convexity of the $L^{2}$-norm. This is enough to pass to the limit in the nonlinearities of the fluid-structure system. Note that with the above and (6.14) we also obtain compactness in $L^{2}\left(I, W^{2, p}(\omega)\right)$ for some $p>2$ and recall from Sect. 1.2 that the energy $K$ is continuous on $W^{2, p}(\omega)$.

In order to complete the proof of Theorem 2.6, we have to pass to the limit in the term $M \nabla_{\mathbf{x}} \mathbf{u}^{\varrho_{n}} \widehat{\psi}^{\varrho_{n}}$ appearing in the Fokker-Planck equation. For this purpose we have to prove compactness of $\widehat{\psi}^{\varrho_{n}}$. For this, we first note that (6.3) and (6.4) imply

$$
\eta^{\varrho_{n}} \rightarrow \eta \quad \text { in } \quad C^{\alpha}(\bar{I} \times \omega)
$$


as $n \rightarrow \infty$ for some $\alpha \in(0,1)$. Therefore, by using

$$
\left\|\mathfrak{r}^{\varrho_{n}} \eta^{\varrho_{n}}-\eta\right\|_{C^{\alpha}(\bar{I} \times \omega)} \leq\left\|\mathfrak{r}^{\varrho_{n}}\left(\eta^{\varrho_{n}}-\eta\right)\right\|_{C^{\alpha}(\bar{I} \times \omega)}+\left\|\mathfrak{r}^{\varrho_{n}} \eta-\eta\right\|_{C^{\alpha}(\bar{I} \times \omega)},
$$

we also get that

$$
\mathfrak{r}^{\varrho_{n}} \eta^{\varrho_{n}} \rightarrow \eta \quad \text { in } \quad C^{\alpha}(\bar{I} \times \omega)
$$

as $n \rightarrow \infty$. Next, similarly to (4.42), we obtain from (6.2) the bound

$$
\sup _{t \in I} \int_{\mathbb{R}^{3} \times B} \chi_{\Omega_{\mathrm{r}} \varrho_{\eta} \eta^{\varrho n}(t)} M \widehat{\psi}^{\varrho_{n}} \ln \left(\mathrm{e}+\widehat{\psi}^{\varrho_{n}}\right) \mathrm{d} \mathbf{q} \mathrm{d} \mathbf{x} \lesssim 1
$$

which holds uniformly in $n \in \mathbb{N}$ and thus, $\widehat{\psi}^{\varrho_{n}}$ is equi-integrable. Similarly to (4.43), we obtain

$$
\chi_{\Omega_{\mathfrak{r}} \varrho_{n_{\eta} \varrho_{n}(t)}} \widehat{\psi}^{\varrho_{n}} \rightarrow \chi_{\Omega_{\eta(t)}} \psi \text { a.e. in } I \times \mathbb{R}^{3} \times B .
$$

for a possible subsequence. If we now combine (6.29) with (6.28), then we can conclude from Vitali's convergence theorem that

$$
\widehat{\psi}^{\varrho_{n}} \rightarrow^{\eta} \psi \quad \text { in } \quad L^{1}\left(I \times \Omega_{\mathfrak{r}} \varrho_{n} \eta^{\varrho_{n}(t)} ; L_{M}^{1}(B)\right)
$$

and because of (6.10), we obtain by interpolation,

$$
\widehat{\psi}^{\varrho_{n}} \rightarrow{ }^{\eta} \psi \quad \text { in } \quad L^{q}\left(I \times \Omega_{\mathfrak{r}_{\varrho_{n}} \eta^{\varrho_{n}(t)}} ; L_{M}^{1}(B)\right) \text { for all } q \in[1, \infty)
$$

and hence

$$
\nabla_{\mathbf{x}} \mathbf{u}^{\varrho_{n}} \widehat{\psi}^{\varrho_{n}} \rightarrow{ }^{\eta} \nabla_{\mathbf{x}} \mathbf{u} \widehat{\psi} \quad \text { in } \quad L^{1}\left(I \times \Omega_{\mathfrak{r} \varrho_{n} \eta^{\varrho_{n}}(t)} ; L_{M}^{1}\left(B ; \mathbb{R}^{3 \times 3}\right)\right)
$$

due to (6.7). The proof of Theorem 2.6 is complete.

Open Access This article is licensed under a Creative Commons Attribution 4.0 International License, which permits use, sharing, adaptation, distribution and reproduction in any medium or format, as long as you give appropriate credit to the original author(s) and the source, provide a link to the Creative Commons licence, and indicate if changes were made. The images or other third party material in this article are included in the article's Creative Commons licence, unless indicated otherwise in a credit line to the material. If material is not included in the article's Creative Commons licence and your intended use is not permitted by statutory regulation or exceeds the permitted use, you will need to obtain permission directly from the copyright holder. To view a copy of this licence, visit http://creativecommons.org/licenses/by/4.0/.

\section{References}

Barrett, J.W., Süli, E.: Existence of global weak solutions to some regularized kinetic models for dilute polymers. Multiscale Model. Simul. 6(2), 506-546 (2007) 
Barrett, J.W., Süli, E.: Existence of global weak solutions to dumbbell models for dilute polymers with microscopic cut-off. Math. Models Methods Appl. Sci. 18(6), 935-971 (2008)

Barrett, J.W., Süli, E.: Existence and equilibration of global weak solutions to kinetic models for dilute polymers I: finitely extensible nonlinear bead-spring chains. Math. Models Methods Appl. Sci. 21(6), 1211-1289 (2011)

Barrett, J.W., Süli, E.: Existence and equilibration of global weak solutions to kinetic models for dilute polymers II: Hookean-type models. Math. Models Methods Appl. Sci. 22(5), 1150,024-84 (2012a)

Barrett, J.W., Süli, E.: Existence of global weak solutions to finitely extensible nonlinear bead-spring chain models for dilute polymers with variable density and viscosity. J. Differ. Equ. 253(12), 3610-3677 (2012b)

Barrett, J.W., Süli, E.: Finite element approximation of finitely extensible nonlinear elastic dumbbell models for dilute polymers. ESAIM Math. Model. Numer. Anal. 46(4), 949-978 (2012c)

Barrett, J.W., Schwab, C., Süli, E.: Existence of global weak solutions for some polymeric flow models. Math. Models Methods Appl. Sci. 15(6), 939-983 (2005)

Bird, R.B., Curtiss, C.F., Armstrong, R.C., Hassager, O.: Dynamics of Polymeric Liquids. Vol. 2: Kinetic Theory. Wiley, New York (1987)

Bodnár, T., Galdi, G.P., Nečasová, Š.: Fluid-Structure Interaction and Biomedical Applications. Advances in Mathematical Fluid Mechanics. Birkhäuser/Springer, Basel (2014)

Boulakia, M., Guerrero, S., Takahashi, T.: Well-posedness for the coupling between a viscous incompressible fluid and an elastic structure. Nonlinearity 32(10), 3548-3592 (2019)

Breit, D., Mensah, P.R.: Local well-posedness of the compressible FENE dumbbell model of Warner-type. arXiv preprint arXiv:1911.02465v2 (2018, to appear in Nonlinearity)

Breit, D., Schwarzacher, S.: Compressible fluids interacting with a linear-elastic shell. Arch. Ration. Mech. Anal. 228(2), 495-562

Bulíček, M., Málek, J., Süli, E.: Existence of global weak solutions to implicitly constituted kinetic models of incompressible homogeneous dilute polymers. Commun. Partial Differ. Equ. 38(5), 882-924 (2013)

Chakrabarti, S.K.: The Theory and Practice of Hydrodynamics and Vibration, vol. 20. World scientific, Singapore (2002)

Chambolle, A., Desjardins, B., Esteban, M.J., Grandmont, C.: Existence of weak solutions for the unsteady interaction of a viscous fluid with an elastic plate. J. Math. Fluid Mech. 7(3), 368-404 (2005)

Ciarlet, P.G.: An Introduction to Differential Geometry with Applications to Elasticity. Springer, Dordrecht (2005). (Reprinted from J. Elasticity 78/79 (2005))

Ciarlet, P.G., Roquefort, A.: Justification of a two-dimensional nonlinear shell model of Koiter's type. Chin. Ann. Math. Ser. B 22(2), 129-144 (2001)

Constantin, P.: Nonlinear Fokker-Planck Navier-Stokes systems. Commun. Math. Sci. 3(4), 531-544(2005)

Constantin, P., Fefferman, C., Titi, E.S., Zarnescu, A.: Regularity of coupled two-dimensional nonlinear Fokker-Planck and Navier-Stokes systems. Commun. Math. Phys. 270(3), 789-811 (2007)

Dowell, E.H.: A Modern Course in Aeroelasticity, Solid Mechanics and Its Applications, Vol. 217, enlarged edn. Springer, Cham (2015)

E, W., Li, T., Zhang, P.: Well-posedness for the dumbbell model of polymeric fluids. Commun Math. Phys. 248(2), 409-427 (2004)

El-Kareh, A.W., Leal, L.G.: Existence of solutions for all deborah numbers for a non-Newtonian model modified to include diffusion. J. Non-Newtonian Fluid Mech. 33(3), 257-287 (1989)

Granas, A., Dugundji, J.: Fixed Point Theory. Springer Monographs in Mathematics. Springer, New York (2003)

Grandmont, C.: Existence of weak solutions for the unsteady interaction of a viscous fluid with an elastic plate. SIAM J. Math. Anal. 40(2), 716-737 (2008)

Gwiazda, P., Lukáčová-Medvidová, M., Mizerová, H., Świerczewska-Gwiazda, A.: Existence of global weak solutions to the kinetic Peterlin model. Nonlinear Anal. Real World Appl. 44, 465-478 (2018)

Hundertmark-Zaušková, A., Lukáčová-Medvid’ová, M., Nečasová, Š: On the existence of weak solution to the coupled fluid-structure interaction problem for non-Newtonian shear-dependent fluid. J. Math. Soc. Japan 68(1), 193-243 (2016)

Ignatova, M., Kukavica, I., Lasiecka, I., Tuffaha, A.: Small data global existence for a fluid-structure model. Nonlinearity 30(2), 848-898 (2017)

Jourdain, B., Lelièvre, T., Le Bris, C.: Existence of solution for a micro-macro model of polymeric fluid: the FENE model. J. Funct. Anal. 209(1), 162-193 (2004) 
Klainerman, S., Majda, A.: Singular limits of quasilinear hyperbolic systems with large parameters and the incompressible limit of compressible fluids. Commun. Pure Appl. Math. 34(4), 481-524 (1981)

Lee, J.M.: Introduction to Smooth Manifolds, Graduate Texts in Mathematics, vol. 218, second edn. Springer, New York (2013)

Lengeler, D.: Globale existenz für die interaktion eines Navier-Stokes-fluids mit einer linear elastischen schale. Ph.D. thesis, Universität (2011)

Lengeler, D., Růžička, M.: Weak solutions for an incompressible Newtonian fluid interacting with a Koiter type shell. Arch. Ration. Mech. Anal. 211(1), 205-255 (2014)

Li, T., Zhang, H., Zhang, P.: Local existence for the dumbbell model of polymeric fluids. Commun. Partial Differ. Equ. 29(5-6), 903-923 (2004)

Lions, P.L., Masmoudi, N.: Global solutions for some Oldroyd models of non-Newtonian flows. Chin. Ann. Math. Ser. B 21(2), 131-146 (2000)

Lions, P.L., Masmoudi, N.: Global existence of weak solutions to some micro-macro models. C. R. Math. Acad. Sci. Paris 345(1), 15-20 (2007)

Lukáčová-Medvid'ová, M., Mizerová, H., Nečasová, Š, Renardy, M.: Global existence result for the generalized Peterlin viscoelastic model. SIAM J. Math. Anal. 49(4), 2950-2964 (2017)

Luo, W., Yin, Z.: Global existence and well-posedness for the FENE dumbbell model of polymeric flows. Nonlinear Anal. Real World Appl. 37, 457-488 (2017)

Masmoudi, N.: Well-posedness for the FENE dumbbell model of polymeric flows. Commun. Pure Appl. Math. 61(12), 1685-1714 (2008)

Masmoudi, N.: Global existence of weak solutions to the FENE dumbbell model of polymeric flows. Invent. Math. 191(2), 427-500 (2013)

Muha, B.: A note on the trace theorem for domains which are locally subgraph of a Hölder continuous function. Netw. Heterog. Media 9(1), 191-196 (2014)

Muha, B., Čanić, S.: Existence of a weak solution to a nonlinear fluid-structure interaction problem modeling the flow of an incompressible, viscous fluid in a cylinder with deformable walls. Arch. Ration. Mech. Anal. 207(3), 919-968 (2013)

Muha, B., Čanić, S.: Existence of a solution to a fluid-multi-layered-structure interaction problem. J. Differ. Equ. 256(2), 658-706 (2014)

Muha, B., Schwarzacher, S.: Existence and regularity for weak solutions for a fluid interacting with a non-linear shell in 3d. arXiv preprint arXiv:1906.01962 (2019)

Nägele, P., Růžička, M., Lengeler, D.: Functional setting for unsteady problems in moving domains and applications. Complex Var. Elliptic Equ. 62(1), 66-97 (2017)

Otto, F., Tzavaras, A.E.: Continuity of velocity gradients in suspensions of rod-like molecules. Commun. Math. Phys. 277(3), 729-758 (2008)

Peralta, G., Kunisch, K.: Analysis of a nonlinear fluid-structure interaction model with mechanical dissipation and delay. Nonlinearity 32(12), 5110-5149 (2019)

Renardy, M.: An existence theorem for model equations resulting from kinetic theories of polymer solutions. SIAM J. Math. Anal. 22(2), 313-327 (1991)

Roquefort, A.: Sur quelques questions liées aux modéles non linéaires de coques minces. Ph.D. thesis, Paris $6(2001)$

Zhang, H., Zhang, P.: Local existence for the FENE-dumbbell model of polymeric fluids. Arch. Ration. Mech. Anal. 181(2), 373-400 (2006)

Publisher's Note Springer Nature remains neutral with regard to jurisdictional claims in published maps and institutional affiliations. 